\title{
ARL2BP, a novel ciliopathy protein, is required for cilia microtubule formation
}

\author{
Abigail Ruth Moye \\ West Virginia University, auhayes@mix.wvu.edu
}

Follow this and additional works at: https://researchrepository.wvu.edu/etd

Part of the Biological Phenomena, Cell Phenomena, and Immunity Commons, Congenital, Hereditary, and Neonatal Diseases and Abnormalities Commons, Disease Modeling Commons, Eye Diseases Commons, Medical Cell Biology Commons, and the Medical Molecular Biology Commons

\section{Recommended Citation}

Moye, Abigail Ruth, "ARL2BP, a novel ciliopathy protein, is required for cilia microtubule formation" (2018). Graduate Theses, Dissertations, and Problem Reports. 3712.

https://researchrepository.wvu.edu/etd/3712

This Dissertation is protected by copyright and/or related rights. It has been brought to you by the The Research Repository @ WVU with permission from the rights-holder(s). You are free to use this Dissertation in any way that is permitted by the copyright and related rights legislation that applies to your use. For other uses you must obtain permission from the rights-holder(s) directly, unless additional rights are indicated by a Creative Commons license in the record and/ or on the work itself. This Dissertation has been accepted for inclusion in WVU Graduate Theses, Dissertations, and Problem Reports collection by an authorized administrator of The Research Repository @ WVU.

For more information, please contact researchrepository@mail.wvu.edu. 


\title{
ARL2BP, a novel ciliopathy protein, is required for cilia microtubule formation
}

\author{
Abigail R. Moye \\ Dissertation submitted to the School of \\ Medicine at West Virginia University \\ in partial fulfillment of the requirements for the \\ degree of \\ Doctor of Philosophy in \\ Biochemistry and Molecular Biology \\ Visvanathan Ramamurthy, Ph.D., Chair \\ Michael Schaller, Ph.D. \\ David Smith, Ph.D. \\ Peter Stoilov, Ph.D. \\ Peter Mathers, Ph.D. \\ Department of Biochemistry and Molecular Biology
}

Morgantown, West Virginia

2018

Keywords: ARL2BP, ARL2, ARL3, photoreceptor, cilia, microtubules, Retinal Degeneration Copyright 2018 Abigail Moye 


\title{
ABSTRACT \\ ARL2BP, a novel ciliopathy protein, is required for cilia microtubule formation
}

\begin{abstract}
Abigail R. Moye
Cilia are specialized organelles essential for cellular function. Not surprisingly, mutations in ciliarelated genes are linked to multi-syndromic diseases termed ciliopathies. These include blinding diseases such as retinitis pigmentosa (RP). One such novel gene is ARL2BP (ARL2-binding protein) and is linked to RP and situs inversus (organ reversal) in humans, a phenotype produced by defects in the nodal cilia of developing embryos. Defects in photoreceptor cilia, as well as situs inversus in human patients, suggest that ARL2BP plays an invaluable role in the structure and function of cilia. However little is known about the role for this protein in the cilium. In this dissertation, I investigate the role for ARL2BP in cilia formation and function using a novel mouse model lacking ARL2BP, generated using CRISPR-Cas9 system. In Chapter 1 of my dissertation, I review the independent role for ARL2BP in cilia. In Chapter 2, the visual phenotype associated with loss of ARL2BP is described. I propose a model in which ARL2BP is required for the proper doublet microtubule structure that comprises the ciliary axoneme. In Chapter 3, I present my recent work demonstrating ARL2BP's role in other ciliated tissues. My findings show that loss of ARL2BP causes an impairment in spermiogenesis, which was also observed in a human patient. In addition, the absence of ARL2BP is linked to situs inversus and ventriculomegaly. In Chapter 4, unpublished results are discussed, revealing the interacting partner of ARL2BP, and how this interaction fits into the regulation of doublet microtubule structure and maintenance. This work provides a strong groundwork for future investigations on ARL2BP and its involvement with its partners and cilia. These experiments will shed light on the function of ARL2BP and ultimately contribute to our understanding of cilia development, photoreceptor structure, and associated human diseases.
\end{abstract}




\section{Acknowledgements}

The work presented in this dissertation would not have been possible without the support and guidance of many individuals including my mentors, colleagues, and friends.

First and foremost, I want to thank my amazing husband, Jesse Moye. I probably would not have even finished my first year of graduate school without you. You have given me more support and encouragement than I could ever have hoped for. You also continue to inspire me, and push me to strive for more. You have also given me the best gift of all, our daughter Penelope, whose joy and love have made it possible to finish this last year of school!

I would next like to thank my mentor, Dr. Visvanathan Ramamurthy. Working with you as an advisor has allowed me to learn so much and branch out into areas of research completely independent of the labs'. Your mentorship, and continual development of independence, is what has given me the opportunity to pursue a future scientific career. You have also provided me with unbelievable mentorship in lab and life, which I hope to be able to emulate throughout my scientific career.

Next, I would like to thank all my lab members and the members in the Eye Institute for advice, encouragement, and blunt counsel throughout the years. I also would not have enjoyed my time here without our lab coffee breaks, lunches, girls nights, and general friendship. I especially want to thank the girls in the lab, Tanya, Daniella, Rawaa, and Emily, for your friendship and help in making me a better person, more professional labmate, and allowing me to grow into a more analytical thinker while also getting excited about science with me.

I would like to thank the Biochemistry Department, and my friends outside of the department that have lended their minds and hands every time I wanted to branch out and learn something new. Special thanks to Dr. Peter Stoilov, Dr. Elena Pugacheva, and Dr. Peter Mathers for their help and support to the advancement of my knowledge and technical skills.

I would like to thank my friends who have supported me, put up with me during my pregnancy, and celebrated with me since. Tayvia - my grad school friend from before we even started, you have always been there for me when I needed it, and I am so glad I had someone to enjoy all the fantasy things with (even if you did ruin that one episode where you find out Angels secret...haha); Jessica - even though our friendship is recent, it has been amazing to have a friend like you (and Chris), and I am so happy we got to be apart of your marriage!; Kim - our twinny friendship provided excitement and fun into my life when I really needed it, and your intellectual prowess and progression into your career has helped motivate me to finally finish!

Lastly, I want to thank my family. They have been the best role-models and given me more support and encouragement throughout my entire life than I ever could have asked for. I thank my sister for being the best friend I could have asked for and the continual inspiration and encouragement for being a mom, my brother for always inspiring me to learn and stay curious about the world (and for being the first person to explain what a science article actually is and what a scientific researcher actually does), and importantly, I cannot imagine how I would have graduated without my parents (Mark and Rhonda) help, sacrifice, love, and hard-work, 
especially this past year with Penelope. I truly don't know how we will survive Houston without you!

And for my daughter, Penelope, for when you pretend to read this whole thing when you're older - you have given me more joy and love than I could ever imagine, and have made my last year of graduate school the most crazy yet amazing year yet. As unintuitive as it sounds, I could not have done it without you!

If anyone was left out of these acknowledgements, please know that I am grateful for your help! 


\section{Table of contents}

$\begin{array}{lll}\text { ABSTRACT } & \text { II }\end{array}$

ACKNOWLEDGEMENTS III

CHAPTER 1: LITERATURE REVIEW 1

THE INDEPENDENT ROLE FOR ARL2BP IN CILIOGENESIS $\quad 1$

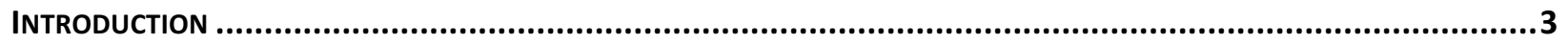

ARL2

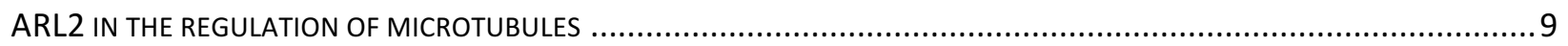

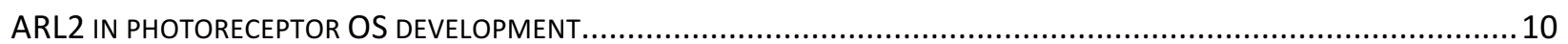

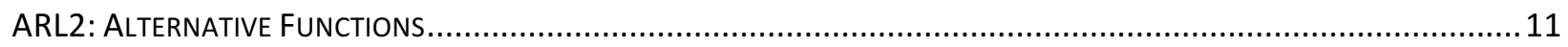

ARL2BP

DISCOVERY AND INTERACTION WITH ARL2

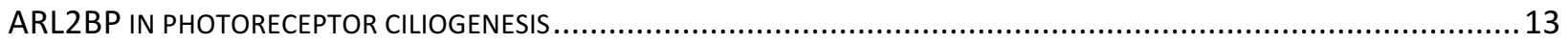

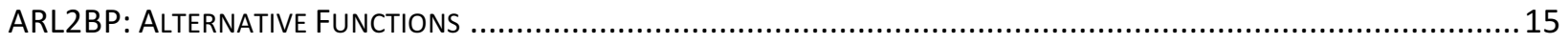

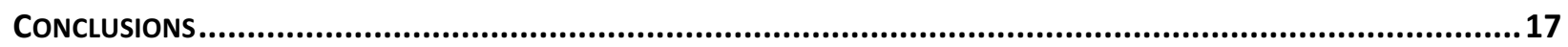

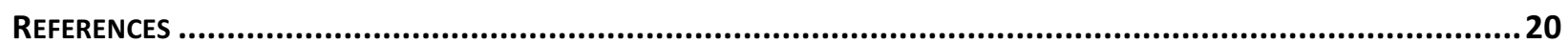

$\begin{array}{lr}\text { CHAPTER } 2 & 25\end{array}$

ARL2BP, A PROTEIN LINKED TO RETINITIS PIGMENTOSA, IS NEEDED FOR NORMAL PHOTORECEPTOR CILIA DOUBLETS AND OUTER SEGMENT STRUCTURE.

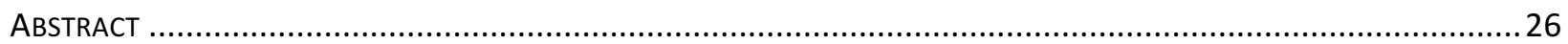

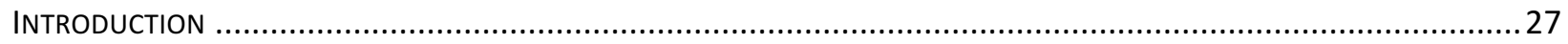

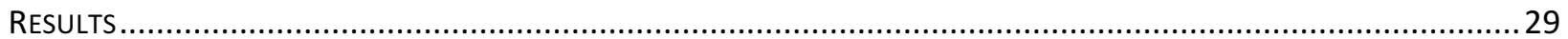

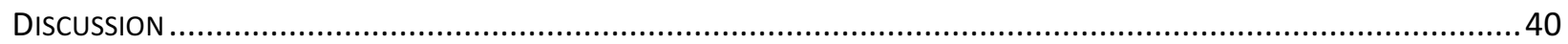

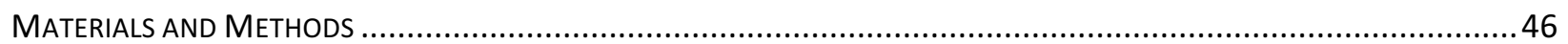

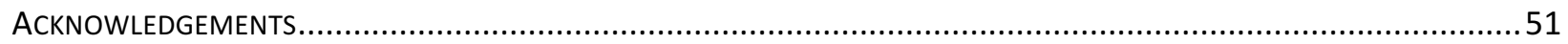

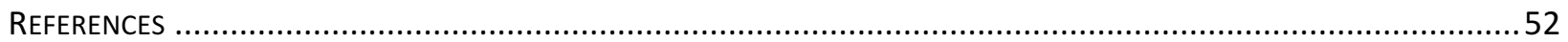

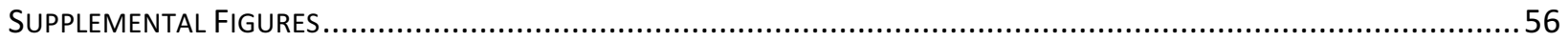

$\begin{array}{lr}\text { CHAPTER } 3 & \mathbf{7 0}\end{array}$

MUTATIONS IN ARL2BP CAUSE SYNDROMIC INFERTILITY IN HUMANS AND MICE.

ABSTRACT

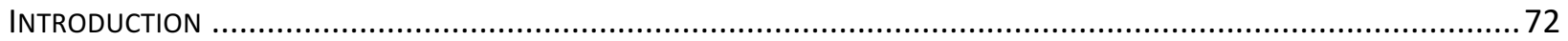

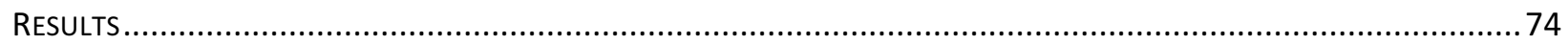

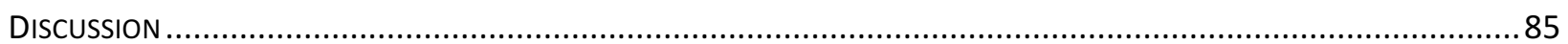

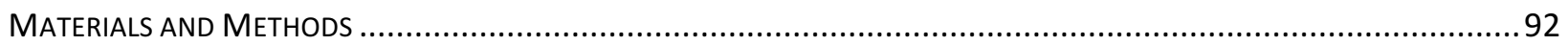

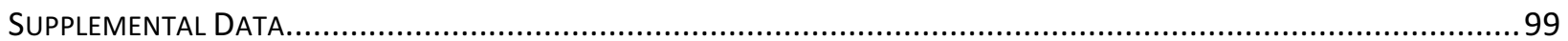

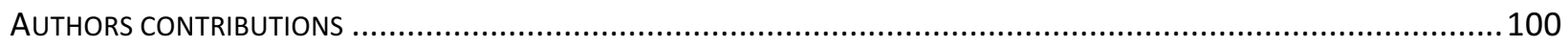

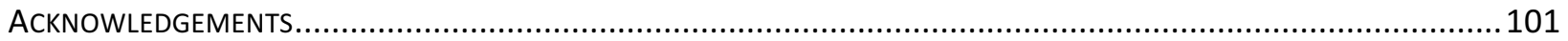

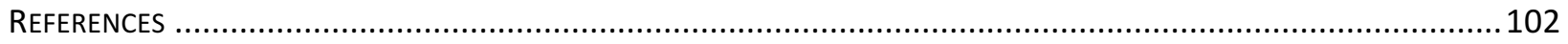

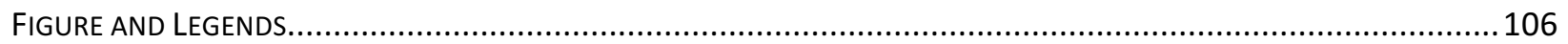




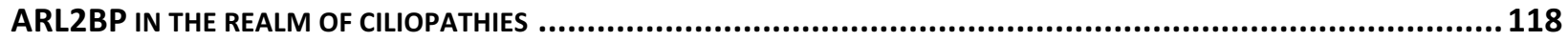

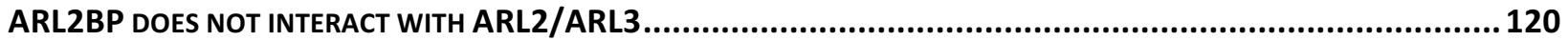

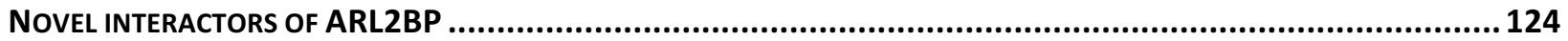

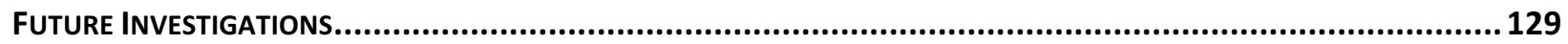

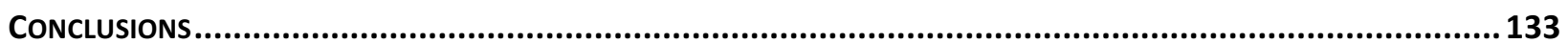

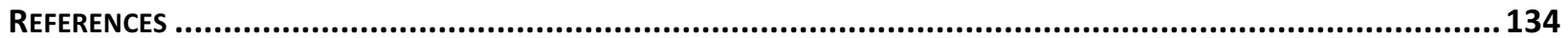




\section{Chapter 1: Literature Review}

The independent role for ARL2BP in ciliogenesis

Abigail R. Moye 


\begin{abstract}
Cilia are tiny, protruding organelles that impart unique functions in cells throughout the body. Two highly characterized cilia, the photoreceptor cell outer segment and the sperm tail flagellum, are functionally and structurally diverse yet are linked through a group of diseases termed ciliopathies. The photoreceptor cell is a complex organelle optimized for capturing light and transducing that signal to downstream neurons. This specialized cilium requires a regulated series of interactions to produce a functional outer segment with organized membrane discs packed with phototransduction proteins. Likewise, the sperm tail flagellum requires tremendous regulation during spermatid maturation to produce a fully functional sperm tail with properly assembled periaxonemal structures. There is growing evidence that small GTPases, such as ADP-Ribosylation Factor Like protein 2 (ARL2) and its related protein ARL2 Binding Partner (ARL2BP), play a major role in the development and maintenance of cilium. Here we review the recent advances in our understanding of ARL2 and ARL2BP underlying the processes of photoreceptor sensory cilium and sperm tail flagellum formation. Importantly, ARL2BP has been implicated in retinal degenerative diseases and syndromic ciliopathies. Understanding the basic mechanisms through which these proteins act will provide insight into pathological states that will guide therapeutic intervention.
\end{abstract}




\section{Introduction}

Cilia are tiny, hair-like organelles that possess fundamental responsibilities in the cell. These protruding organelles are often referred to as "signaling hubs" and are involved in diverse functional roles, including sensation of our environment (hair and photoreceptor cells), fluid movement (trachea, brain, and the embryonic node), and in signaling pathways like Sonic Hedgehog (Levin et al., 1995; Bujakowska et al., 2017; Satir, 2017; Morthorst et al., 2018; Youn and Han, 2018). Though present in multiple cell types, they each retain a highly characterized structure of microtubule protofilaments. In general, motile cilia are considered to possess a $9+2$ microtubule architecture, along with additional structures needed for motility (Figure 1). Immotile cilia possess a 9+0 microtubule architecture, lacking the central pair of microtubules and the corresponding additional structures (Figure 1). Each of the "9" tubules undergo minute changes through each region of the cilium, which are represented by the basal body (BB), transition zone (TZ), and axoneme. In the BB, each tubule is comprised of triplet microtubules, whereas in the $\mathrm{TZ}$ and axoneme, one tubule is lost and they become doublet microtubules (DMT). At the distal end of the axoneme, they undergo loss of one more tubule and become singlets (Figure 1). Furthermore, each of the 9 DMTs are formed by an A- and B-tubule, each possessing 14 or 10 microtubule protofilaments, respectively. The A-tubule is characterized by a ring structure, and is the tubule that continues on in the singlet region, and the B-tubule is characterized by a c-shape which joins the A-tubule at two places, the inner and outer junction (Nicastro et al., 2011; Linck et al., 2014). The inner junction (IJ) also contains an $11^{\text {th }}$ nontubulin protofilament, which is thought to be made by the proteins Flagella Associated protein 20 (FAP20), Parkin Coregulated Gene Protein (PACRG), and Tektin (Ikeda et al., 2007; Yanagisawa et al., 2014). 


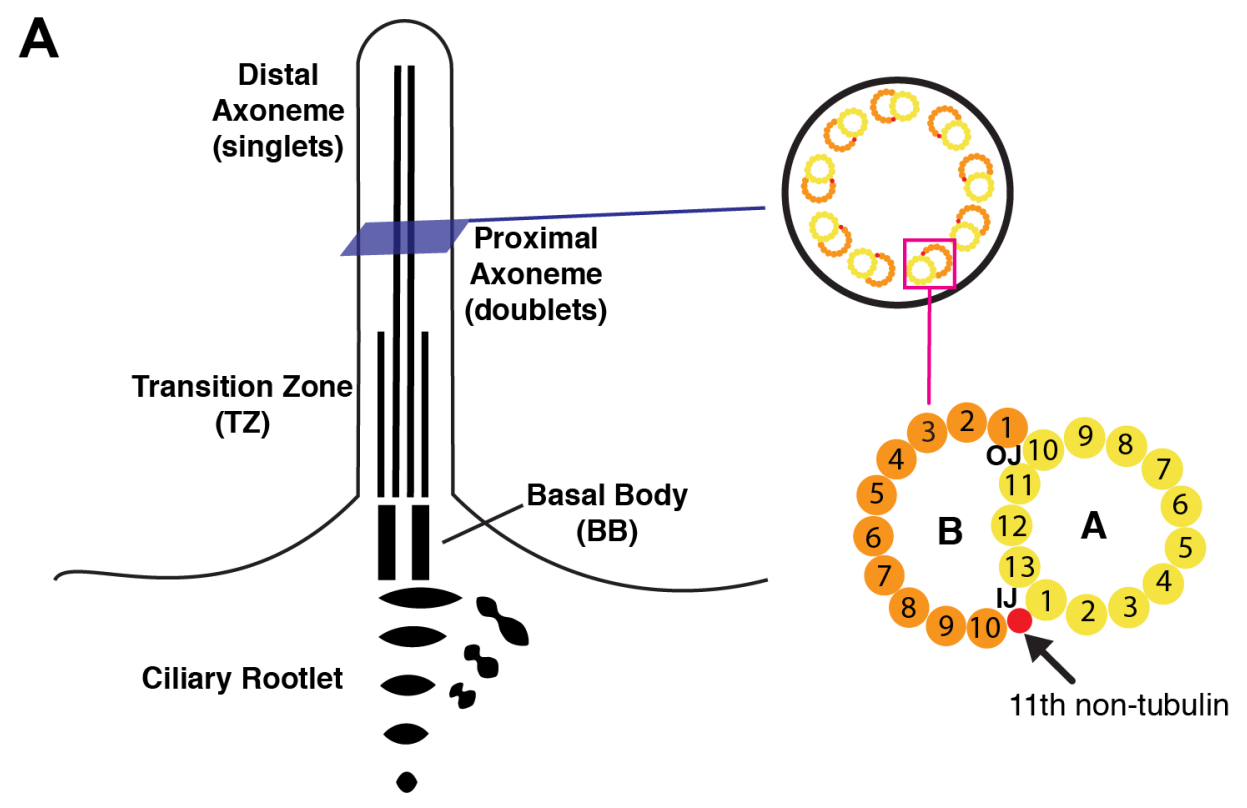

Supplemental Figure 1. Scheme of cilia structure.

An illustration of a primary cilium, with all the regions annotated. To the right is a crosssectional view of the proximal axoneme displaying doublet microtubules $(9+0)$. One of these doublet microtubules is further zoomed in to display the protofilament structure of the A- and Btubules.

Photoreceptor (PR) cells are light-sensing post-mitotic neurons that relay light stimuli to the rest of the retina and on to the brain (Figure 2). These cells are highly specialized and utilize a complex modified cilium, the photoreceptor outer segment (OS), to efficiently capture light (Pearring et al., 2013) (Figure 2). Photoreceptors pose major demands in regulation of developmental processes such as OS formation as well as maintenance of function by selective trafficking of OS proteins to their site of action. This need is exacerbated considering that the outer segment goes through a daily process of shedding and renewal, so that every 10 days the entire OS is renewed (Young, 1967). Cilia formation, i.e. ciliogenesis, in photoreceptor cells must be efficiently regulated to allow for creation of a functional, organized outer segment (OS) with stacked membrane discs that are packed with the phototransduction and structural proteins necessary for sight. The demand for efficient protein trafficking in the mature OS is a result of 
the anatomy of the photoreceptor cell which has distinct compartments, i.e. the inner segment (IS) where OS proteins are synthesized and the OS where those proteins must function to capture light (Nachury et al., 2010) (Figure 2). Correspondingly, human disease may result from inefficiency in the process of protein trafficking in photoreceptor cells. Notably, mutations that disrupt the transport of phosphodiesterase 6 (PDE6), rhodopsin, or other phototransduction proteins are a major cause of the blinding disease, retinitis pigmentosa (RP) (Wang et al., 2005; Iannaccone et al., 2006). RP alone is responsible for vision loss in 1 in 4,000 people worldwide (Sung and Chuang, 2010).

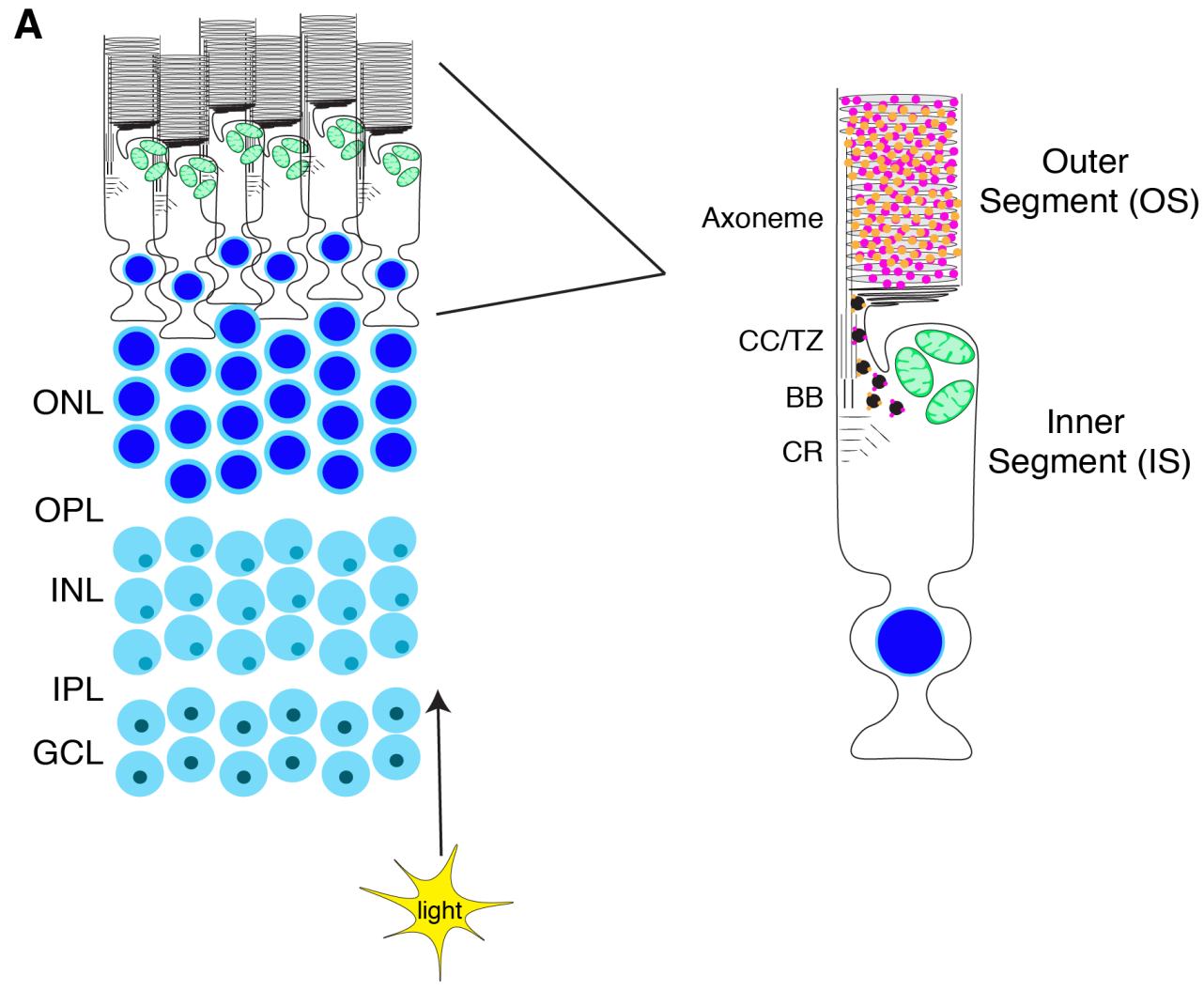

\section{Supplemental Figure 2. Scheme of photoreceptor structure.}

The laminated structure of the retina, dispaying the layers of retinal neurons and the direction of incoming light. To the right is a magnified scheme of the photoreceptor cells, demonstrating the compartmentalization of the OS and IS, and the requirement of the cilium for disc morphogenesis and protein trafficking. $\mathrm{ONL}=$ outer nuclear layer; $\mathrm{INL}=$ inner nuclear layer; $\mathrm{OPL}=$ outer plexiform layer; IPL=inner plexiform layer; $\mathrm{GCL}=$ ganglion cell layer. 
Importantly however, several blinding diseases, such as RP, are the result of malformation of the OS and these diseases often occur because of an inherited mutation in the genes involved in ciliary formation. These defects can result in blinding disease or in syndromic disorders known as ciliopathies, the result of a generalized defect in cilia of multiple organs.

One cell type often affected in ciliopathy patients is the sperm flagellum. Like photoreceptors, sperm possess a highly specialized structure required for proper function. The sperm tail flagellum, however, contains additional accessory structures not present in other cilia. Spermatogenesis, the development and maturation of sperm, begins in the basal compartment at the outer edge of the seminiferous tubules of the testes. The sperm cells develop and mature in a series of stages progressing inward toward the lumen of the seminiferous tubules. As spermatids mature, they are released into the lumen and transported to the epididymal tissues. The last stages of spermatogenesis are characterized by tail formation, i.e. spermiogenesis, beginning with axoneme growth and assisted by a transient microtubule- and F-actin-based structure called the manchette (Oakberg, 1956) (Figure 3B). The fully developed sperm tail consists of four regions, including the connecting piece, the mid piece, the principal piece, and the end piece (Figure 3A). The additional structures associated with the axoneme differ throughout these segments, with a mitochondrial sheath (MS) surrounding the mid piece, and a fibrous sheath (FS) surrounding the principal piece. The MS and FS surround 9 outer dense fibers (ODFs) that correspond to the 9 doublets of the axoneme, except when ODFs 3 and 8 are replaced by 2 longitudinal columns of the fibrous sheath (Oko, 1989). In the endpiece of the sperm flagellum these periaxonemal structures are shed, leaving only the microtubular axoneme (Figure 3A). 


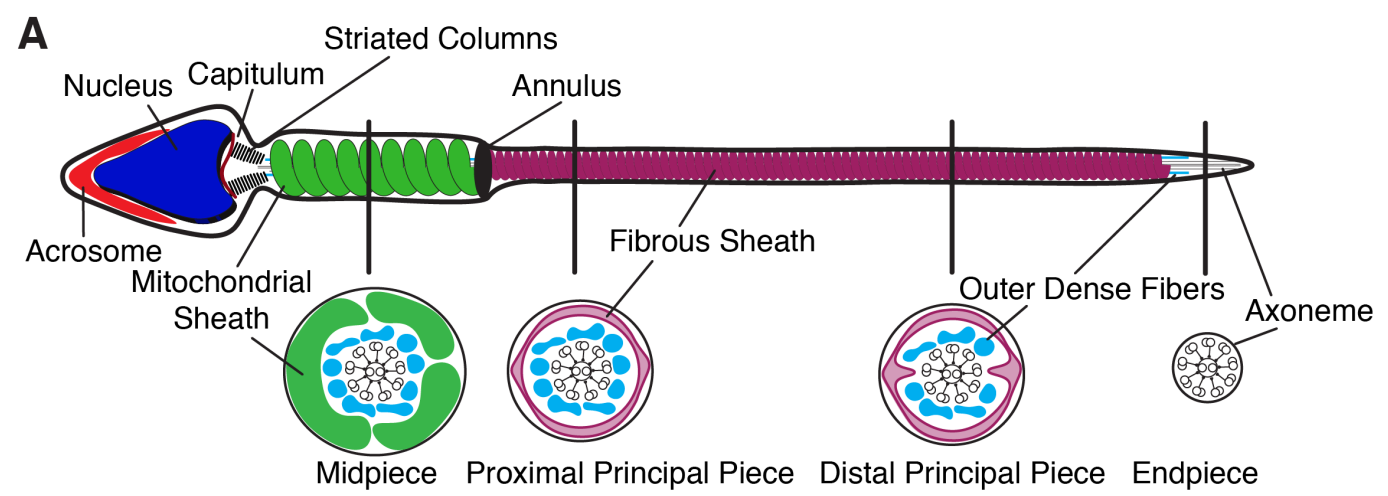

B

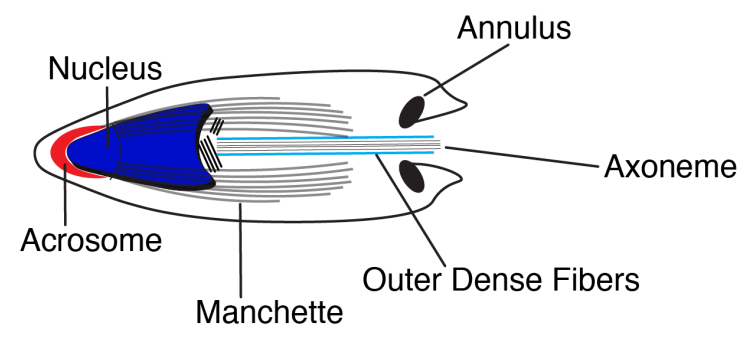

\section{Supplemental Figure 3. Scheme of sperm structure.}

A. Scheme illustrating the four regions of the mature sperm tail, the mid piece, the principal piece, and the end piece. The additional structures associated with the axoneme are the mitochondrial sheath, fibrous sheath, and outer dense fibers. B. Scheme illustrating the sperm during tail formation with presence of the manchette, required for assembly of the periaxonemal structures and the acrosome.

Despite the importance of both of these uniquely modified cilia, the sperm flagellum and the photoreceptor OS, the mechanisms behind development of these structures are poorly understood. The aim of this review is to discuss the emerging role of the proteins ARL2 and ARL2BP in regards to photoreceptor and sperm cell biology. 


\section{ARL2}

ARL2, ADP-Ribosylation Factor Like 2, is a small GTPase, which are a major family of proteins that are regulated by accessory proteins that control their activity by nucleotide binding and release (Figure 4). This feature, along with the localization of accessory proteins and/or GTPases within a specialized compartment, makes them ideal for functioning in specific spatiotemporal conditions. Small GTPases are also known regulators of protein and vesicular trafficking as well as developmental processes in other cell types. However, most small GTPases, like ARL2, are understudied in the realm of photoreceptor cell biology, and will be discussed here.

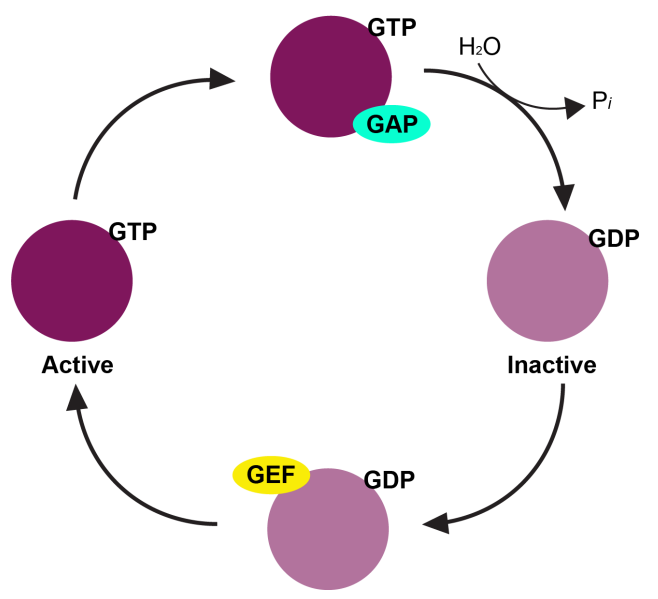

Figure 4. Scheme of small GTPase nucleotide states.

Scheme illustrating the switch of small GTPases from GTP-bound (active form) to GDP-bound (inactive form) through the help of guanine nucleotide exchange factors (GEF-helps exchange GDP for GTP) or GTPase activating proteins (GAP - helps hydrolyze the GTP to form GDP). 


\section{ARL2 in the regulation of microtubules}

The Meinke group in 2000 showed that the ARL2 homolog, TITAN5 in Arabidopsis, was involved in cytoskeleton rearrangements during embryo and endosperm development (McElver et al., 2000). Later that year, Antoshechkin and $\operatorname{Han}^{6}$ showed that defects in the C. elegans homolog to ARL2, evl20, also produced enlarged or multiple nuclei in embryos, indicative of failed cytokinesis. They were also able to rescue this phenotype with expression of human ARL2 (Antoshechkin and Han, 2002). ARL2 has been implicated in many additional processes associated with microtubule and tubulin dynamics and these functions appear to be mediated at centrosomes, one of the places where ARL2 is localized (Bhamidipati et al., 2000; Zhou et al., 2006; Tian et al., 2010; Mori and Toda, 2013). More recent studies have teased out a mechanism through which ARL2 may affect microtubule dynamics in vivo. Specifically, ARL2-GTP interacts with TBCD and TBCE to form the "TBC-DEG" complex which is then able to catalytically manipulate $\alpha \beta$-tubulin dimers driving potentially both biogenesis and degradation of those dimers by modulation of the ARL2 GTPase activity. It is thought that the energy needed to dissociate $\alpha \beta$-tubulin dimers or release soluble polymerizable $\alpha \beta$-tubulin dimers is supplied by the GTP hydrolysis of ARL2 that is facilitated by one of its' potential GAPs, tubulin binding cofactor C (TBCC) (Nithianantham et al., 2015).

Conversely, the Kahn group has proposed a different mechanism for ARL2/TBCD regulation of tubulin, by identifying a trimer consisting of ARL2/TBCD/ß-tubulin. This interaction was assessed in multiple mammalian tissues and cell lines through non-denaturing gels. They were able to purify TBCE with this trimer as well, but only at sub-stoichiometric levels, and they were not able to show a direct interaction with TBCC. The ARL2/TBCD/ $\beta$ - 
tubulin was shown to affect polymerized microtubule densities, with disruption of ARL2-TBCD interaction correlating with decreased polymerized microtubules (Francis et al., 2017).

A third group has shown a combination of these results, with TBCC showing a binding preference for ARL2-GTP, and TBCE showing a binding preference for ARL2-GDP (Chen et al., 2016). Furthermore, the interaction between ARL2 and TBCD was validated in vivo through retinal lysates expressing ARL2-Q70L (unpublished data). These conclusions demonstrate that ARL2 is involved in regulating the soluble pool of $\alpha$ - and $\beta$-tubulin and, therefore, the dynamics of microtubules that use that tubulin. They also reveal the possibility of a dynamic process in which ARL2-GTP/TBCD/ $\beta$-tubulin complex interacts with TBCE/ $\alpha$-tubulin complex to form $\alpha \beta$-tubulin dimers, and with a decrease or increase in ARL2 hydrolysis causing a disruption in the highly dynamic polymerized microtubules (Figure 3) (Francis et al., 2016).

\section{ARL2 in photoreceptor OS development}

Due to the importance of ARL2 in many cellular processes, total ablation causes embryonic lethality. Therefore, the use of transgenic murine models have yielded significant insight into the roles of ARL2 in vivo. One of the models studied the role for ARL2 in photoreceptor cells through over-expression of the dominant-active form of ARL2 (ARL2Q70L) (Wright et al., 2018). These animals exhibited problems in the development of both the inner and outer segments of photoreceptor cells. Furthermore, the ciliary axoneme appeared shorter with constitutively-active ARL2. This result is not only in accordance with the ARL2Q70L related reduction in polymerized microtubules observed by (Nithiananatham et al., 2015; Francis et al., 2017), but also demonstrates a role for ARL2 in development of cilia. In conclusion, ARL2-Q70L is crucial for photoreceptor neuron sensory cilium development and it specifically functions to regulate the process of ciliogenesis. 


\section{ARL2: Alternative Functions}

\section{Mitochondria}

ARL proteins typically possess an N-terminal glycine residue that is modified by a myristoyl lipid group (Kahn et al., 1988; Randazzo and Kahn, 1995; Lee et al., 1997). However, it was shown that ARL2 and ARL3 are not myristoylated, and may therefore be involved in cellular processes that do not require membrane localization (Sharer et al., 2002a). Instead, the $\mathrm{N}$-terminus of ARL2 is key to its mitochondrial targeting, and has been shown to localize within the intermembrane space (IMS) of mitochondria and to interact with an inner membrane protein, adenine nucleotide transporter (ANT1) (Sharer et al., 2002b). Although it was later shown that the majority of ARL2 was cytosolic and only about $10 \%$ was present in the IMS, it was confirmed that ARL2 has a role in mitochondria (Sharer et al., 2002a). Mitochondrial biochemical assays and live cell imaging revealed that loss of ARL2, through knockdown or use of a dominant-negative ARL2 (ARL2-T30N), caused mitochondrial fragmentation and loss of mitochondrial motility. Conversely, over-activation of ARL2 (ARL2-Q70L) caused no mitochondrial fragmentation, but instead caused a defect on microtubules (Newman et al., 2014). This is in accordance with the absence of mitochondrial defects observed with expression of ARL2-Q70L in photoreceptors, a cell-type extremely reliant on mitochondria (Wright et al., 2018). The Kahn group further demonstrated that loss of the ARL2 GAP, ELMOD2, also resulted in mitochondrial fragmentation and loss of mitochondrial motility (Newman et al., 2014; Newman et al., 2017). Furthermore, they demonstrated a partial rescue of mitochondrial fragmentation in MFN2-/- MEFs (a mitochondrial fusion protein) with expression of ARL2Q70L. They also observed an increase in ARL2 and ELMOD2 levels within the mitochondria of MFN2-/- MEFs (Newman et al., 2017). 
Additionally, knockdown of ARL2, but neither reduction in ELMOD2 nor expression of dominant-ARL2 mutants, resulted in a 50\% reduction in cellular ATP production (Newman et al., 2014). Importantly, it was also demonstrated that there were no microtubule defects with knockdown of ARL2, which contradicts data from S. cerevisiae and S. pombe (Radcliffe et al., 2000; Mori and Toda, 2013). Therefore, though it can be concluded that ARL2 has an obvious role in mitochondrial fusion and fission, through its' GAP ELMOD2, ARL2's role in ATP regulation is unclear. These results indicate that ARL2 function in the mitochondria may increase during times of mitochondrial stress, but otherwise, the main function of ARL2 is involved in tubulin regulation.

\section{ARL2BP}

ARL2BP, ADP-Ribosylation Factor Like 2 Binding Partner, is a relatively small protein thought to be an effector protein of ARL2. Very little on the function of ARL2BP was known until recently, which will be discussed here.

\section{Discovery and interaction with ARL2}

As suggested by its name, ARL2BP is a binding protein of ARL2, although the functional aspects of this interaction remain unclear. The ability of ARL2 to bind GTP in the absence of lipids is what permitted the discovery of ARL2BP. In 1999, Sharer, et. al. performed a GTPase overlay assay to discover potential effectors of ARL2 and ARL3 (Sharer and Kahn, 1999). In this assay, GTP-bound ARL2 specifically bound to ARL2BP on the membrane. In contrast, GDP-ARL2 was not able to detect ARL2BP (Sharer and Kahn, 1999). The binding specificity 
and affinity were confirmed by in vitro assays, and the dissociation constant for GTP-ARL2ARL2BP was between 10-20nM.

In 2009 the crystal structure of ARL2BP-GTP-ARL2 was published, displaying a unique effector-GTPase binding interaction, confirmed through mutagenesis and in vitro binding assays (Zhang et al., 2009a). They show that the LLXIL motif of ARL2 inserts into a hydrophobic pocket of ARL2BP while $\alpha 3$ helix of ARL2BP interacts with the switch region in ARL2. This type of interaction was also shown between ARL3 and one of its' effectors CCDC104/BARTL1 (Lokaj et al., 2015).

Though multiple ex vivo interaction assays have recently been performed on ARL2 (Francis et al., 2017), investigation of the interaction between ARL2 and ARL2BP has not been explored further. Native page analysis of ARL2 displays a band at 40kDa, which could be ARL2 and ARL2BP, but this band was not discussed or analyzed further (Francis et al., 2017). The assays to prove interaction of ARL2 with ARL2BP in vivo have been fruitless, including immunoprecipitation and mass spectrometry (data not shown, Chapter 4). Though it is not clear if the ARL2-ARL2BP interaction has any physiological significance, phenotypic analysis of the ARLSBP -/- model will provide insight into its putative role with ARL proteins.

\section{ARL2BP in photoreceptor ciliogenesis}

In 2013, it was found that mutations in ARL2 binding protein (ARL2BP, a.k.a. Binder of ARL Two (BART)) cause an autosomal-recessive form of retinitis pigmentosa. ARL2BP was localized to the basal body and cilium-associated centriole of photoreceptor cells as well as the periciliary ridge region (Davidson et al., 2013; Moye et al., 2018). Davidson et al. (2013) implicated both ARL2BP and ARL2 in the process of ciliogenesis by showing that knockdown 
of either gene by siRNA in ARPE19 cells results in shortened cilia. Additionally, one of the ARL2BP mutations associated with RP, p.Met45Arg amino acid substitution, results in a significant decrease in the binding affinity to ARL2, suggesting that loss of this interaction is potentially responsible for the RP phenotype in these patients (Davidson et al. 2013). Furthermore, ARL2BP localization to the basal body was lost in ARPE19 cell lines after depletion of ARL2 and in patient epithelial cell lines with the p.Met45Arg mutation (Davidson et al. 2013). These data suggest that the interaction of ARL2 and ARL2BP is necessary for proper localization to the basal body. However, it is unclear whether ARL2BP or ARL2 has a major function associated with basal body or centriolar dynamics and whether one or the other is simply necessary for proper localization. Interestingly, in a murine knockout model of ARL2BP, ARL2 did not appear to be affected with loss of ARL2BP, making their interaction in vivo elusive (data not shown). Nevertheless, this animal model was used to investigate the role for ARL2BP in photoreceptor ciliogenesis. Loss of ARL2BP resulted in shortened axonemes, dysmorphic outer segment disc structure, and cell death (Moye et al., 2018). Furthermore, ARL2BP was shown to be critical for the formation of the doublet microtubule structure of ciliary axonemes, similar to the phenotype displayed in nodal cilia expressing the Henin ARL13B mutant (Caspary et al., 2007). Interestingly, since loss of ARL13B produces a more critical defect on photoreceptor OS than ARL2BP (discs do not form vs discs form vertically and still produce an electrical response, (Hanke-Gogokhia et al., 2017; Dilan, et. al. 2018; in revision), the loss of B-tubule inner junction closure, though critical for stability and growth of the axoneme and outer segment, is not necessary for OS disc formation. These results provide further insight into what proteins and pathways are required for OS disc formation. 


\section{ARL2BP: Alternative Functions}

Node

During early embryogenesis, the node in vertebrates is responsible for organization of the embryo during gastrulation. At this early phase of gastrulation, left/right-patterning of the visceral organs is determined (Nonaka et al., 1998; Komatsu and Mishina, 2013). The two cell types present in the node, pit and crown, are monociliated cells that possess a $9+0$ arrangement of microtubules in their cilia (Hashimoto et al., 2010). Normally, this corresponds to what was thought of as immotile cilia, but it has been proven that some of these cilia are motile and are responsible for the leftward fluid flow in the node that determines L/R asymmetry (Supp et al., 1997; Yoshiba et al., 2012). Intriguingly, only 2-3 functioning cilia are needed to maintain leftward fluid flow and proper organ placement (Nonaka et al., 1998; Nonaka et al., 2002), yet shortened cilia, no matter how many, create a vortical flow, which affects the L/R axis (Bonnafe, 2004).

ARL2BP KO mice display laterality defects, situs inversus and heterotaxia (Chapter 3), and one patient with a mutation in ARL2BP also displayed situs inversus (Davidson et al., 2013). It is possible that ARL2BP is also required for length maintenance in the nodal cilia, like it appears to be for photoreceptor cilia. But further investigations of the nodal cilia in ARL2BP KO mice are required.

Sperm

We have recently shown that ARL2BP is required for spermiogenesis in mice and humans, and that loss of ARL2BP results in immotility and shortened sperm tails (Chapter 3). It was found that without ARL2BP, the periaxonemal structures, such as the mitochondrial sheath (MS), fibrous sheath (FS), and outer dense fibers (ODF) do not assemble properly. This is associated with a defect in the microtubular axoneme structure, where no doublets were found 
and microtubule protofilaments are in complete disarray with no $9+0$ arrangement. The effect loss of ARL2BP has on sperm flagellar assembly appears even more severe than in photoreceptor cilia, which did develop the 9+0 microtubule axoneme. Photoreceptors and sperm both have a highly segmented structure and order of assembly, but sperm tail biogenesis relies on an extra microtubule-based structure, the manchette, for proper formation and assembly of all the periaxonemal structures. It is possible that loss of ARL2BP results in manchette disruptions as well as impairments in axoneme formation, which causes the more drastic phenotype observed in sperm compared to photoreceptor cells.

Since ARL2BP exhibits functions in cilia from multiple tissues, delineating the role for ARL2BP will provide significant insight into cilia structure and function. Furthermore, considering that there are no other published mouse models displaying defects in the doublet microtubule structure, or mouse models of microtubule structural proteins (microtubule interacting proteins, MIPs), conducting research on ARL2BP and its relationship with cilia will be invaluable. Likewise, ARL2BP's lack of effect on intraflagellar transport grants a unique model system to study cilia formation in separation of IFT. Lastly, the study of ARL2BP in photoreceptors and spermiogenesis will impart novel insight into the fields of photoreceptor and sperm cell biology. 


\section{Conclusions}

A comparison of the role for ARL2 demonstrates a unique case of divergence in function of similar ARL proteins. With 53\% sequence identity and 63\% similarity to ARL3, for instance, as well as a number of common binding proteins, it is hard to imagine that ARL2 has unique functions. However, minor differences in the way ARL2 or ARL3 interacts with their shared binding factors, as well as interactions with unique binding partners, likely decides the functional fate. For example, ARL3 and ARL2 both interact directly with carrier protein UNC119, which is thought to regulate trafficking of myristoylated cargo, however, the outcome of this interaction is different. Specifically, binding of ARL3-GTP to an UNC119-Cargo complex results in a conformational change in UNC119 that causes release of the cargo. However, using this same experimental paradigm ARL2-GTP cannot stimulate release of cargo (Wright et al., 2011). This demonstrates that although similar small GTPases may have common interacting partners and a high degree of sequence homology they do not necessarily confer the same functional relationship.

It is also important to note that ARL2BP does not currently have a proposed role in the ARL2/TBCD/Tubulin models. Though in vivo studies have shown that ARL2BP colocalizes with ARL2 in the pericentriolar matrix (PCM) of centrosomes, which could indicate their interaction, overexpression or loss of ARL2BP does not cause the defects in tubulin polymerization or centrosomal fragmentation that occur upon expression of dominant active ARL2-Q70L (Zhou et al., 2006). It should be noted that these results are specific to centrosomes and not cilia-associated centrioles. This suggests that ARL2BP and ARL2 may have exclusive co-dependent functions in cilia-associated centrioles only during specific stages of cell development. Furthermore, though ARL2BP was also shown to bind ANT-1 with ARL2 in the 
IMS of mitochondria, knockdown of ARL2BP did not cause any mitochondrial defect (Sharer et al., 2002a; Newman et al., 2014), and KO of ARL2BP in mice did not cause any phenotypes associated with mitochondrial dysfunction (Moye et al., 2018). This implies that ARL2BP plays no mitochondrial role. Intriguingly, however, due to a recent paper proposing that ARL2 contains a mitochondrial targeting sequence in its' N-terminus (Sharer et al., 2002a), and the knowledge that this N-terminus of ARL2 binds to a hydrophobic pocket of ARL2BP (Zhang et al., 2009b), it is plausible to consider that ARL2BP could be involved in targeting ARL2 to basal bodies/cilia, aiding in the dual roles ARL2 possesses. To fully understand their interaction in the context of the photoreceptor cell basal body, ciliary associated centriole, connecting cilium, and OS, their interaction needs to be characterized in ciliated cell lines completely.

Though ARL2BP

was initially identified as an effector for ARL2, it is

clear that the primary

function of ARL2BP within the cell is independent from ARL2 or ARL3. Based on the defects associated with ARL2BP KO mice, and patients with mutations in ARL2BP, it appears that ARL2BP has a unique role in axoneme assembly and
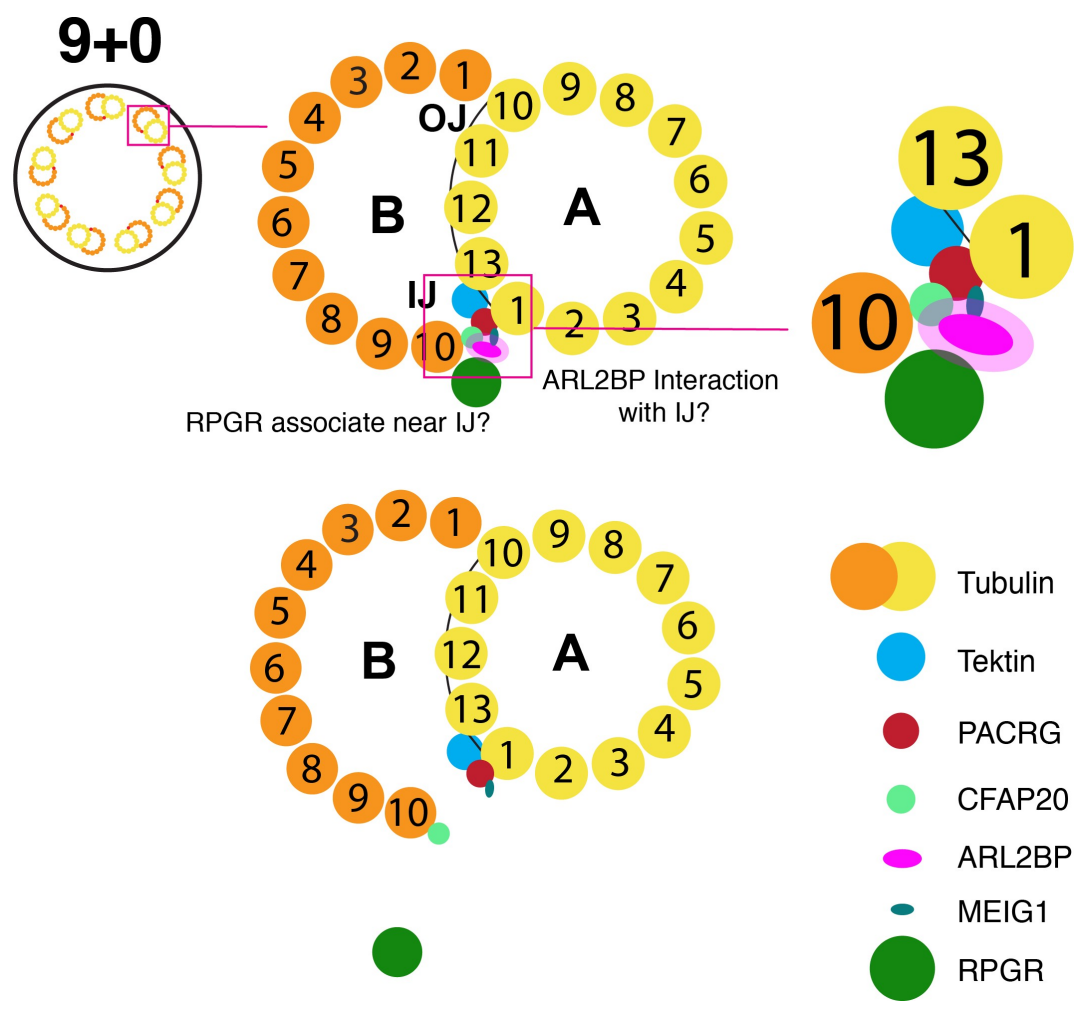

Figure 1. Model for the putative role of ARL2BP.

$9+0$ cilia cross-section structure. WT on top. ARL2BP may interact with any of the inner junction proteins of doublet microtubules (light pink indicates possible interaction of ARL2BP). Without ARL2BP (bottom), IJ is open with the resident proteins exposed, and RPGR is mislocalized. 
doublet microtubule formation. Based on work described in Chlamydomonas, there are three proteins found in and around the inner junction of microtubule doublets, CFAP20, PACRG, and Tektin (Ikeda et al., 2007; Yanagisawa et al., 2014). It is possible that the main role for ARL2BP is through interactions with these proteins. ARL2BP could be providing support in these proteins' interaction with the microtubules or proper localization of these proteins to the cilium (Figure 3). Alternatively, since mutations in ARL13B in embryonic nodes also result in an open inner junction of doublet microtubules, ARL2BP could be interacting with ARL13B to regulate axoneme assembly. However, this is less likely since ARL13B-/- is much more severe than the ARL2BP mouse model. Additionally, a pull-down using retinal and testis extracts did not indicate an interaction between ARL13B and ARL2BP (Chapter 4). 


\section{References}

Antoshechkin, I., and Han, M. (2002). The C-elegans evl-20 gene is a homolog of the small GTPase ARL2 and regulates cytoskeleton dynamics during cytokinesis and morphogenesis. Dev Cell 2, 579-591.

Bhamidipati, A., Lewis, S.A., and Cowan, N.J. (2000). ADP ribosylation factor-like protein 2 (Ar12) regulates the interaction of tubulin-folding cofactor D with native tubulin. Journal of Cell Biology 149, 1087-1096.

Bonnafe, E., Touka, M., AitLounis, A., Baas, D., Barras, E., Ucla, C., Moreau, A., Flamant, F., Dubruille, R., Couble, P., Collingnon, J., Durand, B., and Reith, W. (2004). The Transcription Factor RFX3 Directs Nodal Cilium Development and Left-Right Asymmetry Specification 24, 4417-4427.

Bujakowska, K.M., Liu, Q., and Pierce, E.A. (2017). Photoreceptor Cilia and Retinal Ciliopathies. Cold Spring Harb Perspect Biol 9.

Caspary, T., Larkins, C.E., and Anderson, K.V. (2007). The Graded Response to Sonic Hedgehog Depends on Cilia Architecture. Dev Cell 12, 767-778.

Chen, K., Koe, C.T., Xing, Z.B., Tian, X., Rossi, F., Wang, C., Tang, Q., Zong, W., Hong, W.J., Taneja, R., Yu, F., Gonzalez, C., Wu, C., Endow, S., and Wang, H. (2016). Arl2- and Mspsdependent microtubule growth governs asymmetric division. The Journal of Cell Biology, jcb.201503047-jcb.201503047.

Davidson, A.E., Schwarz, N., Zelinger, L., Stern-Schneider, G., Shoemark, A., Spitzbarth, B., Gross, M., Laxer, U., Sosna, J., Sergouniotis, P.I., Waseem, N.H., Wilson, R., Kahn, R.a., Plagnol, V., Wolfrum, U., Banin, E., Hardcastle, A.J., Cheetham, M.E., Sharon, D., and Webster, A.R. (2013). Mutations in ARL2BP, encoding ADP-ribosylation-factor-like 2 binding protein, cause autosomal-recessive retinitis pigmentosa. American Journal of Human Genetics 93, 321329.

Francis, J.W., Newman, L.E., Cunningham, L.A., and Kahn, R.A. (2017). A Trimer Consisting of the Tubulin-specific Chaperone D (TBCD), Regulatory GTPase ARL2, and beta-Tubulin Is Required for Maintaining the Microtubule Network. J Biol Chem 292, 4336-4349.

Francis, J.W., Turn, R.E., Newman, L.E., Schiavon, C., and Kahn, R.A. (2016). Higher order signaling: ARL2 as regulator of both mitochondrial fusion and microtubule dynamics allows integration of 2 essential cell functions. Small GTPases 7, 188-196.

Hanke-Gogokhia, C., Wu, Z., Sharif, A., Yazigi, H., Frederick, J.M., and Baehr, W. (2017). The guanine nucleotide exchange factor, Arf-like protein 13b, is essential for assembly of the mouse photoreceptor transition zone and outer segment. Journal of Biological Chemistry.

Hashimoto, M., Shinohara, K., Wang, J., Ikeuchi, S., Yoshiba, S., Meno, C., Nonaka, S., Takada, S., Hatta, K., Wynshaw-Boris, A., and Hamada, H. (2010). Planar polarization of node cells determines the rotational axis of node cilia. Nature cell biology 12, 170-176. 
Iannaccone, A., Man, D., Waseem, N., Jennings, B.J., Ganapathiraju, M., Gallaher, K., Reese, E., Bhattacharya, S.S., and Klein-Seetharaman, J. (2006). Retinitis pigmentosa associated with rhodopsin mutations: Correlation between phenotypic variability and molecular effects. Vision Res 46, 4556-4567.

Ikeda, K., Ikeda, T., Morikawa, K., and Kamiya, R. (2007). Axonemal localization of Chlamydomonas PACRG, a homologue of the human Parkin-coregulated gene product. Cell Motil Cytoskeleton 64, 814-821.

Kahn, R.A., Goddard, C., and Newkirk, M. (1988). Chemical and immunological characterization of the $21-\mathrm{kDa}$ ADP-ribosylation factor of adenylate cyclase. Journal of Biological Chemistry 263, 8282-8287.

Komatsu, Y., and Mishina, Y. (2013). Establishment of left-right asymmetry in vertebrate development: the node in mouse embyros. Cell Mol Life Sci 70, 4659-4666.

Lee, F.-J.S., Huang, C.-F., Yu, W.-L., Buu, L.-M., Lin, C.-Y., Huang, M.-C., Moss, J., and Vaughan, M. (1997). Characterization of an ADP-ribosylation Factor-like 1 Protein inSaccharomyces cerevisiae. Journal of Biological Chemistry 272, 30998-31005.

Levin, M., Johnson, R.L., Stern, C.D., Kuehn, M., and Tabin, C. (1995). A molecular pathway determining left-right asymmetry in chick embryogenesis. Cell 82, 803-814.

Linck, R., Fu, X., Lin, J., Ouch, C., Schefter, A., Steffen, W., Warren, P., and Nicastro, D. (2014). Insights into the structure and function of ciliary and flagellar doublet microtubules: tektins, Ca2+-binding proteins, and stable protofilaments. J Biol Chem 289, 17427-17444.

Lokaj, M., Kösling, Stefanie K., Koerner, C., Lange, Sven M., van Beersum, Sylvia E., van Reeuwijk, J., Roepman, R., Horn, N., Ueffing, M., Boldt, K., and Wittinghofer, A. (2015). The Interaction of CCDC104/BARTL1 with Arl3 and Implications for Ciliary Function. Structure(London, England:1993) 23, 2122-2132.

McElver, J., Patton, D., Rumbaugh, M., Liu, C., Yang, L.J., and Meinke, D. (2000). The TITAN5 gene of Arabidopsis encodes a protein related to the ADP ribosylation factor family of GTP binding proteins. Plant Cell 12, 1379-1392.

Mori, R., and Toda, T. (2013). The dual role of fission yeast Tbc1/cofactor C orchestrates microtubule homeostasis in tubulin folding and acts as a GAP for GTPase Alp41/Arl2. Molecular biology of the cell 24, 1713-1724, S1711-1718.

Morthorst, S.K., Christensen, S.T., and Pedersen, L.B. (2018). Regulation of ciliary membrane protein trafficking and signalling by kinesin motor proteins. The FEBS Journal 0.

Moye, A.R., Singh, R., Kimler, V.A., Dilan, T.L., Munezero, D., Saravanan, T., Goldberg, A.F.X., and Ramamurthy, V. (2018). ARL2BP, a protein linked to retinitis pigmentosa, is needed for normal photoreceptor cilia doublets and outer segment structure. Mol Biol Cell 29, 15901598. 
Nachury, M., Seeley, E., and Jin, H. (2010). Trafficking to the ciliary membrane: how to get across the periciliary diffusion barrier? Annu Rev Cell Dev Bio 26, 59-87.

Newman, L.E., Schiavon, C.R., Zhou, C., and Kahn, R.A. (2017). The abundance of the ARL2 GTPase and its GAP, ELMOD2, at mitochondria are modulated by the fusogenic activity of mitofusins and stressors. PLoS ONE 12, e0175164.

Newman, L.E., Zhou, C.-j., Mudigonda, S., Mattheyses, A.L., Paradies, E., Marobbio, C.M.T., and Kahn, R.A. (2014). The ARL2 GTPase is required for mitochondrial morphology, motility, and maintenance of ATP levels. PloS one 9, e99270-e99270.

Nicastro, D., Fu, X., Heuser, T., Tso, A., Porter, M.E., and Linck, R.W. (2011). Cryo-electron tomography reveals conserved features of doublet microtubules in flagella. Proceedings of the National Academy of Sciences of the United States of America 108, E845-E853.

Nithiananatham, S., Le, S., Seto, E., Jia, W., Leary, J., Corbett, K.D., and Jeffrey, K. (2015). Tubulin cofactors and Arl2 are cage-like chaperones that regulate the soluble $\alpha \beta$-tubulin pool for microtubule dynamics. eLife, 1-33.

Nithianantham, S., Le, S., Seto, E., Jia, W., Leary, J., Corbett, K.D., Moore, J.K., and AlBassam, J. (2015). Tubulin cofactors and Arl2 are cage-like chaperones that regulate the soluble $\alpha \beta$-tubulin pool for microtubule dynamics. eLife 4, e08811.

Nonaka, S., Shiratori, H., Saijoh, Y., and Hamada, H. (2002). Determination of left-right patterning of the mouse embryo by artificial nodal flow. Nature 418, 96-99.

Nonaka, S., Tanaka, Y., Okada, Y., Takeda, S., Harada, A., Kanai, Y., Kido, M., and Hirokawa, N. (1998). Randomization of left-right asymmetry due to loss of nodal cilia generating leftward flow of extraembryonic fluid in mice lacking KIF3B motor protein. Cell 95, 829-837.

Oakberg, E.F. (1956). A description of spermiogenesis in the mouse and its use in analysis of the cycle of the seminiferous epithelium and germ cell renewal. American Journal of Anatomy 99, 391-413.

Oko, R., Clermont, Y. (1989). Light microscope immunocytochemical study of fibrous sheath and outer dense fiber in the rat spermatid. Anat Rec 225, 46-55.

Pearring, J.N., Salinas, R.Y., Baker, S.A., and Arshavsky, V.Y. (2013). Protein sorting, targeting and trafficking in photoreceptor cells. Prog Retin Eye Res 36, 24-51.

Radcliffe, P.A., Vardy, L., and Toda, T. (2000). A conserved small GTP-binding protein Alp41 is essential for the cofactor-dependent biogenesis of microtubules in fission yeast. FEBS Letters $468,84-88$.

Randazzo, P.A., and Kahn, R.A. (1995). [31] Myristoylation and ADP-ribosylation factor function. In: Methods in Enzymology, vol. 250: Academic Press, 394-405.

Satir, P. (2017). CILIA: before and after. Cilia 6, 1. 
Sharer, J.D., and Kahn, R.A. (1999). The ARF-like 2 (ARL2)-binding protein, BART.

Purification, cloning, and initial characterization. J Biol Chem 274, 27553-27561.

Sharer, J.D., Shern, J.F., Valkenburgh, H.V., Wallace, D.C., and Kahn, R.A. (2002a). Arl2 and BART enter mitochondria and bind the adenine nucleotide transporter. Molecular biology of the cell 13, 71-83.

Sharer, J.D., Shern, J.F., Van Valkenburgh, H., Wallace, D.C., and Kahn, R.A. (2002b). ARL2 and BART enter mitochondria and bind the adenine nucleotide transporter. Mol Biol Cell 13, 7183.

Sung, C.-H., and Chuang, J.-Z. (2010). The cell biology of vision. The Journal of cell biology 190, 953-963.

Supp, D.M., Witte, D.P., Potter, S.S., and Brueckner, M. (1997). Mutation of an axonemal dynein affects left-right asymmetry in inversus viscerum mice. Nature 389, 963-966.

Tian, G., Thomas, S., and Cowan, N.J. (2010). Effect of TBCD and its Regulatory Interactor Ar12 on Tubulin and Microtubule Integrity. Cytoskeleton 67, 706-714.

Wang, D.Y., Chan, W.M., Tam, P.O., Baum, L., Lam, D.S., Chong, K.K., Fan, B.J., and Pang, C.P. (2005). Gene mutations in retinitis pigmentosa and their clinical implications. Clin Chim Acta $351,5-16$.

Wright, K.J., Baye, L.M., Olivier-Mason, A., Mukhopadhyay, S., Sang, L., Kwong, M., Wang, W., Pretorius, P.R., Sheffield, V.C., Sengupta, P., Slusarski, D.C., and Jackson, P.K. (2011). An ARL3-UNC119-RP2 GTPase cycle targets myristoylated NPHP3 to the primary cilium. Genes Dev 25, 2347-2360.

Wright, Z.C., Loskutov, Y., Murphy, D., Stoilov, P., Pugacheva, E., Goldberg, A.F.X., and Ramamurthy, V. (2018). ADP-Ribosylation Factor-Like 2 (ARL2) regulates cilia stability and development of outer segments in rod photoreceptor neurons. Sci Rep 8, 16967.

Yanagisawa, H.-a., Mathis, G., Oda, T., Hirono, M., Richey, E.A., Ishikawa, H., Marshall, W.F., Kikkawa, M., and Qin, H. (2014). FAP20 is an inner junction protein of doublet microtubules essential for both the planar asymmetrical waveform and stability of flagella in Chlamydomonas. Molecular Biology of the Cell 25, 1472-1483.

Yoshiba, S., Shiratori, H., Kuo, I.Y., Kawasumi, A., Shinohara, K., Nonaka, S., Asai, T., Sasaki, G., Belo, J.A., Sasaki, H., Nakai, J., Dworniczak, B., Ehrlich, B.E., Pennekamp, P., and Hamada, H. (2012). Cilia at the node of mouse embryos sense fluid flow for left-right determination via Pkd2. Science 338, 226-231.

Youn, Y.H., and Han, Y.-G. (2018). Primary Cilia in Brain Development and Diseases. The American Journal of Pathology 188, 11-22.

Young, R.W. (1967). The renewal of photoreceptor cells outer segments The Journal of Cell Biology 33, 61-72. 
Zhang, T., Li, S., Zhang, Y., Zhong, C., Lai, Z., and Ding, J. (2009a). Crystal structure of the ARL2-GTP-BART complex reveals a novel recognition and binding mode of small GTPase with effector. Structure 17, 602-610.

Zhang, T., Li, S., Zhang, Y., Zhong, C., Lai, Z., and Ding, J. (2009b). Crystal Structure of the ARL2-GTP-BART Complex Reveals a Novel Recognition and Binding Mode of Small GTPase with EffectorZhang T, Li S, Zhang Y, Zhong C, Lai Z, Ding J (2009) Crystal Structure of the ARL2-GTP-BART Complex Reveals a Novel Recognition and B. Structure 17, 602-610.

Zhou, C., Cunningham, L., Marcus, A.I., Li, Y., and Kahn, R.A. (2006). Arl2 and Arl3 regulate different microtubule-dependent processes. Molecular biology of the cell 17, 2476-2487. 


\section{Chapter 2}

ARL2BP, a protein linked to Retinitis Pigmentosa, is needed for normal photoreceptor cilia doublets and outer segment structure.

Abigail R. Moye ${ }^{\text {a,b }}$, Ratnesh Singh ${ }^{\text {b }}$, Victoria A. Kimler ${ }^{d}$, Tanya L. Dilan ${ }^{\text {a,b }}$, Daniella

Munezero $^{\text {a,b }}$, Thamaraiselvi Saravanan ${ }^{\mathrm{a}}$, Andrew F.X. Goldberg ${ }^{\mathrm{d}}$, and Visvanathan Ramamurthy $^{\mathbf{a}, \mathrm{b}, \mathrm{c}}$

Departments of Ophthalmology ${ }^{\mathrm{a}}$, Biochemistry ${ }^{\mathrm{b}}$, and Rockefeller Neurosciences Institute ${ }^{\mathrm{c}}$, West Virginia University, Morgantown, WA 26506, USA

Eye Research Institute ${ }^{\mathrm{d}}$, Oakland University, Rochester, MI 48309, USA

\section{Corresponding Author:}

Visvanathan Ramamurthy, Departments of Ophthalmology and

Biochemistry; Eye Institute, One Medical Center Drive, West Virginia University, Morgantown, WV 26506-9193, USA.

Phone: 304-598-6925

Email: ramamurthyv@wvumedicine.org 


\begin{abstract}
The outer segment (OS) of photoreceptor cells is an elaboration of a primary cilium with organized stacks of membranous discs that contain the proteins needed for phototransduction and vision. Though cilia formation and function has been well characterized, little is known about the role of cilia in the development of photoreceptor OS. Nevertheless, progress has been made by studying mutations in ciliary proteins which often result in malformed outer segments and lead to blinding diseases. To investigate how ciliary proteins contribute to outer segment formation, we generated a knockout mouse model for ARL2BP, a ciliary protein linked to Retinitis Pigmentosa. The knockout mice display an early and progressive reduction in visual response. Prior to photoreceptor degeneration we observed disorganization of the photoreceptor OS, with vertically aligned discs and shortened axonemes. Interestingly, ciliary doublet microtubule structure was also impaired, displaying open B-tubule doublets, paired with loss of singlet microtubules. Based on results from this study, we conclude that ARL2BP is necessary for photoreceptor cilia doublet formation and axoneme elongation, which is required for outer segment morphogenesis and vision.
\end{abstract}




\section{Introduction}

Photoreceptors are ciliated neurons that absorb photons and convert light into electrical signals. These neurons are compartmentalized with outer and inner segments (OS and IS) bridged by a narrow connecting cilium ( $\mathrm{CC}$, corresponds to the ciliary transition zone) with an extended axoneme (Pearring et al., 2013; Goldberg et al., 2016; May-Simera et al., 2017). The OS contains stacked membranous discs that anchor proteins that participate in phototransduction (Molday and Moritz, 2015). Remarkably, photoreceptors shed 10\% of their discs every day (Young, 1967; Goldberg et al., 2016). To maintain the outer segments, photoreceptors need to ensure continuous transport of proteins and membranes from their site of synthesis in the IS through the CC to the OS (Young, 1967). In addition to facilitating protein movement, the photoreceptor axoneme is thought to play a structural role in the formation and continual replacement of OS discs (Liu et al., 2004b).

Though the photoreceptor cilium is highly modified in function, the basic structure is consistent with immotile primary cilia seen in other tissues, containing $9+0$ microtubule morphology that undergoes a switch from doublet microtubules to singlet microtubules approximately one-third of the way up the axoneme (Brown et al., 1963; Steinberg and Wood, 1975; Knabe and Kuhn, 1997; Insinna et al., 2008; Wensel et al., 2016). The axonemal doublet microtubules (DMT) consist of an A-tubule containing 13 tubulin protofilaments joined to a Btubule containing 10 tubulin protofilaments, with an $11^{\text {th }}$ non-tubulin complex linking the inner junction of A and B tubules (Figure 7A) (Linck and Stephens, 2007; Nicastro et al., 2011; Pigino et al., 2012; Linck et al., 2014; Ichikawa et al., 2017). Defects in the structural integrity of photoreceptor CC/axoneme lead to retinal degenerative diseases such as Retinitis Pigmentosa (RP), Lebers Congenital Amaurosis (LCA), and multiple ciliopathies (Pierce et al., 1999; 
Ramamurthy and Cayouette, 2009; Omori et al., 2010; Boldt et al., 2011; Bujakowska et al., 2017). For example, mice lacking Retinitis Pigmentosa 1 (RP1), a protein that links the OS discs to the axoneme, displayed shortened axonemes and disordered OS disc structure (Liu et al., 2003; Liu et al., 2004a). Conversely, the absence of Male Germ-Cell Associated Kinase (MAK) in murine retina resulted in extended axonemes, as well as disorganized OS discs (Omori et al., 2010). Despite the importance of the ciliary axoneme in photoreceptor structure and function, relatively little is known of the mechanism and players behind ciliogenesis and disc organization in the OS.

ADP-Ribosylation Factor-Like 2 Binding Protein (ARL2BP) is a protein present in the CC and basal body (BB) regions of photoreceptor cells (Davidson et al., 2013), however the role for ARL2BP in photoreceptors is unknown. In cultured cells, ARL2BP was found specifically localized to the centrosome and midbody of actively dividing cells (Zhou et al., 2006). Additionally, the knockdown of ARL2BP expression in ARPE-19 cells produced a modest reduction in the length of primary cilia, suggesting a role for ARL2BP in ciliogenesis (Davidson et al., 2013).

There are multiple pathogenic mutations in ARL2BP leading to blindness, one of which is a recessive splice site mutation leading to premature termination of the gene (Davidson et al., 2013). To model RP and determine the role for ARL2BP, we generated a mouse knockout (KO) of ARL2BP using the CRISPR-Cas9 system. Findings from this study corroborate the phenotype evident in patients, displaying photoreceptor dysfunction with loss of ARL2BP. Our data further establishes a photoreceptor-specific role for ARL2BP in axoneme length regulation, disc morphogenesis, and ciliary doublet formation. 


\section{Results}

\section{Generation of ARL2BP knockout mice.}

We used the CRISPR-Cas9 system to create a global knockout (KO) of ARL2BP (Scheme illustrated in Figure. 1A). Three homozygous mouse lines - a 6-bp, a 1-bp, and an 8-bp deletion in ARL2BP - were identified. The 6-bp deletion line produced an in-frame removal of two amino acids ( $\Delta 16-17$ amino acid residues, Figure $1 \mathrm{~B})$. Both 1-bp and 8-bp deletions caused a frame shift mutation and led to a premature termination (Figure 1B). These two lines are reminiscent of one of the patient mutations, splice site variant mutation in ARL2BP, which also caused ablation of the protein (Davidson et al., 2013). The ARL2BP-null mice were comparable in weight and development to WT littermates, with no detectable health issues.

To demonstrate the removal of ARL2BP protein in our 1- and 8-bp deletion animal models, we generated a rabbit antibody against full-length mouse ARL2BP protein. Immunoblot analysis using this antibody and a commercially available monoclonal antibody on retinal lysates at P30 from wild type (WT) and ARL2BP KO littermates confirmed the absence of ARL2BP in KO animals (Figure 1C and Supplemental Figure 1B). For the remainder of this study, unless otherwise stated, ARL2BP KO mice will refer to mice possessing an 8-bp deletion. Localization of ARL2BP in the retina was established using a monoclonal antibody, showing punctate staining of ARL2BP in the IS, BB, and CC of photoreceptors (Figure 1D and E). This is consistent with the localization presented in a published study using immuno-electron microscopy (Scheme illustrated in Figure 1F) (Davidson et al., 2013). The localization of ARL2BP was further confirmed by exogenous expression of subretinally-injected epitope-tagged ARL2BP (Supplemental Figure 1C). 

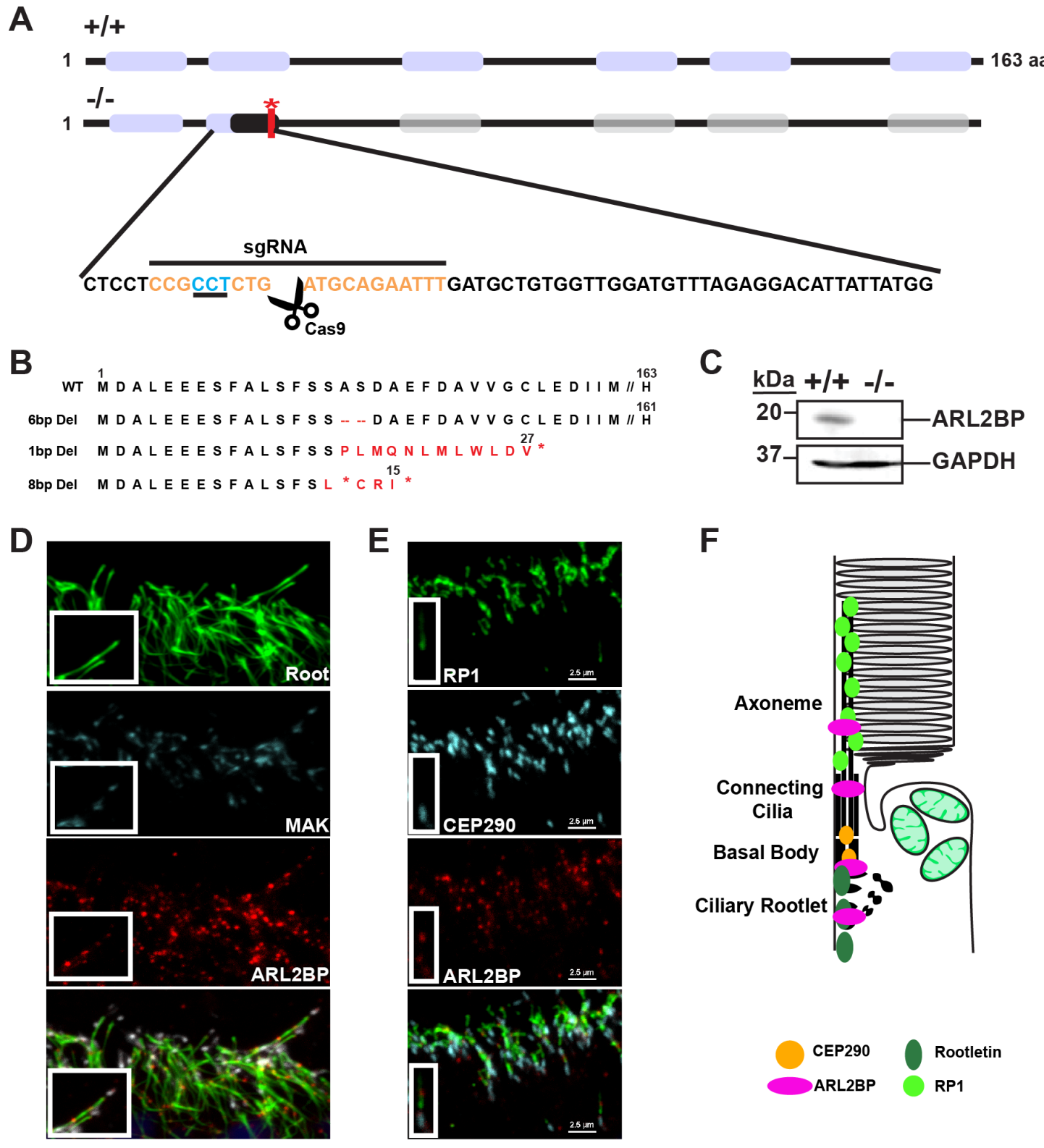

Figure 1. Endogenous ARL2BP expression and generation of knockout model.

(A) Scheme illustrating mutations generated by the Crispr-Cas9 system in ARL2BP. Exon 2 is magnified, showing the sgRNA target locus where Cas 9 generates a double stranded break. The protospacer adjacent motif (PAM) for Cas9 is indicated in blue. (B) Non-homologous end joining (NHEJ) following the Cas9 cleavage led to multiple mutations. In two of the mutants, 1- and 8-base pair deletions (bp del) were found, causing a frameshift mutation and early stop codon (red asterisk). In the third mutant, there was an in-frame 6-bp deletion, producing loss of two amino acids. Red asterisk marks stop in protein. (C) Immunoblot analysis of endogenous ARL2BP protein levels in P16 retinal tissues using ARL2BP and GAPDH (loading control) directed antibodies. Protein molecular weight in kilodaltons $(\mathrm{kDa})$ is indicated on the left. $\mathrm{n}=3$; wildtype $(+/+)$, knockout $(-/-)$. (D and E) Retinal cross sections from wildtype $(+/+)$ mice at P25 showing localization for ARL2BP (red) in relation to (D) Rootletin (Root, green) and Male germ-cell associated kinase (MAK, cyan), and (E) Retinitis Pigmentosa 1 (RP1, green) and Centrosomal protein of 290kDa (CEP290, cyan) (scale bar $=2.5 \mu \mathrm{m}$ ). (F) Scheme illustrating the localization of cilia proteins and ARL2BP in photoreceptors based on the results from panels (D and E). 


\section{ARL2BP is essential for efficient photoresponse.}

Photoreceptor function was assessed in WT and KO littermates using electroretinogram (ERG) recordings, an established technique that measures the hyperpolarization generated by photoreceptor cells upon detection of light (Pinto et al., 2007). Homozygous KO mice displayed a $67 \%$ decrease in rod-derived (scotopic) ERG response ("a"-wave) at the earliest recorded time point, P16, when mice are just opening their eyes (Figure 2A). Rod photoreceptors lacking ARL2BP exhibited a comparable sensitivity to WT at P16 $\left(\mathrm{I}_{1 / 2}=0.2075 \pm 0.02 \mathrm{~cd} . \mathrm{s} \mathrm{m}^{-2}(+/+)\right.$ and $0.241 \pm 0.06 \mathrm{~cd} . \mathrm{s} \mathrm{m}^{-2}(-/-)$, with reduced a-wave maximum amplitudes of $528.4 \mu \mathrm{V} \pm 12.93$ $\mu \mathrm{V}(+/+)$ and $179.8 \mu \mathrm{V} \pm 10.48 \mu \mathrm{V}(-/-)$ (Figure. $2 \mathrm{C}$ ). We observed a progressive loss of function with a $90 \%$ reduction in rod photoresponse by P210 (Figure 2A, D). It is important to

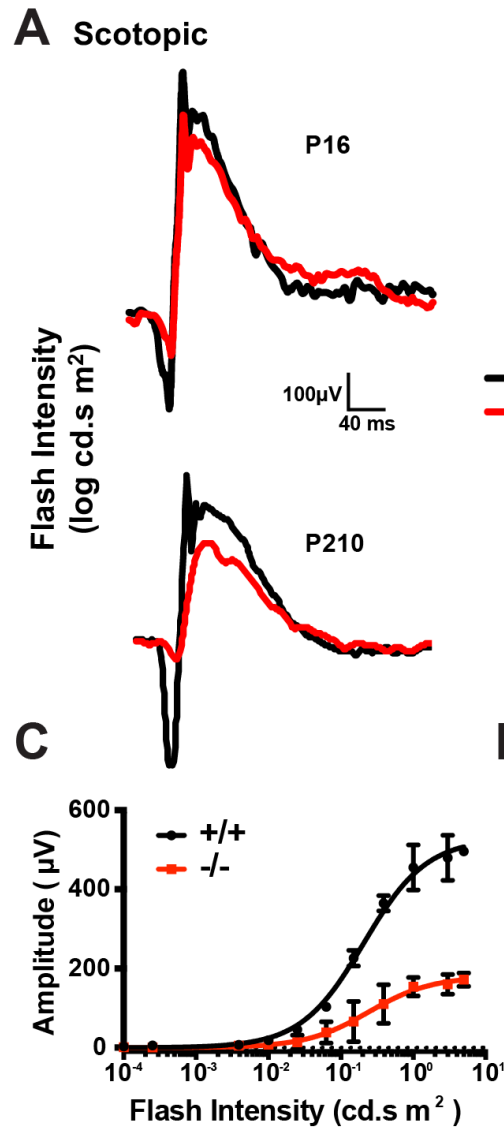

B Photopic
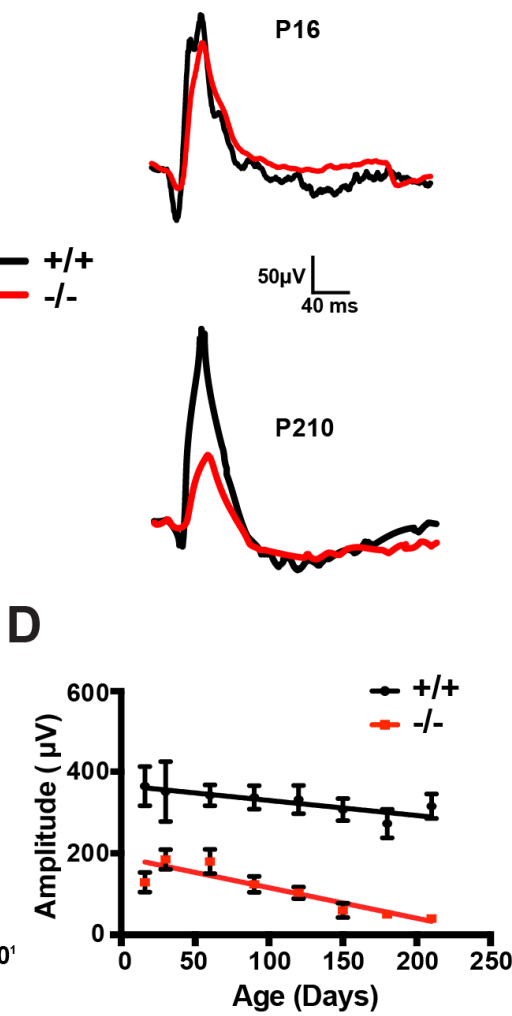

Figure 2. Decreased photoreceptor function in animals lacking ARL2BP. (A and B) Representative scotopic (rod, A) and photopic (cone, B) electroretinograms (ERGs) at $-0.8 \mathrm{~cd} . \mathrm{s} \mathrm{m}-2$ (A) and $0.69 \mathrm{~cd} . \mathrm{s}$ $\mathrm{m}-2$ (B) comparing wildtype $(+/+)$ and knockout (-/-) animals at P16 and P210. (C) Intensity-response relationships for scotopic "a"-waves recorded at P16 between $+/+$ and $-/-$. The data were fitted with a hyperbolic function to define scotopic "a"-wave half-saturating light intensities of $0.2075 \pm 0.02 \mathrm{~cd} . \mathrm{s} \mathrm{m}-2(+/+$, $n=4)$ and $0.241 \pm 0.06$ cd.s m-2 $(-/-, n=4)$ and "a"-wave maximum amplitudes of $528.4 \mu \mathrm{V} \pm 12.93 \mu \mathrm{V}(+/+, \mathrm{n}=4)$ and 179.8 $\mu \mathrm{V} \pm 10.48 \mu \mathrm{V}(-/-, \mathrm{n}=4)$. (D) Scotopic "a"-wave amplitude measured at the light intensity of $-0.8 \mathrm{~cd} . \mathrm{s} \mathrm{m}-2$ across multiple ages between $+/+$ and -/-. Data for (C) and (D) are represented as mean \pm SEM $(n=4$, unpaired two-tailed t-test; all were statistically significant, $P<0.05$ ). All experiments were conducted with littermate controls. 
note that heterozygous mice did not reveal any retinal phenotypes, demonstrating that one WT allele is sufficient for ARL2BP function within the retina.

Similar to rods, cone photoresponses were reduced at P16 and progressively declined as
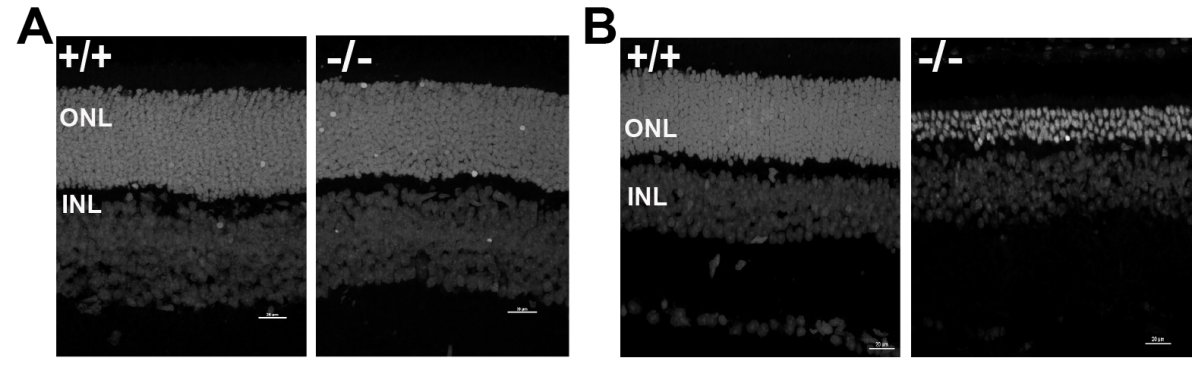

C
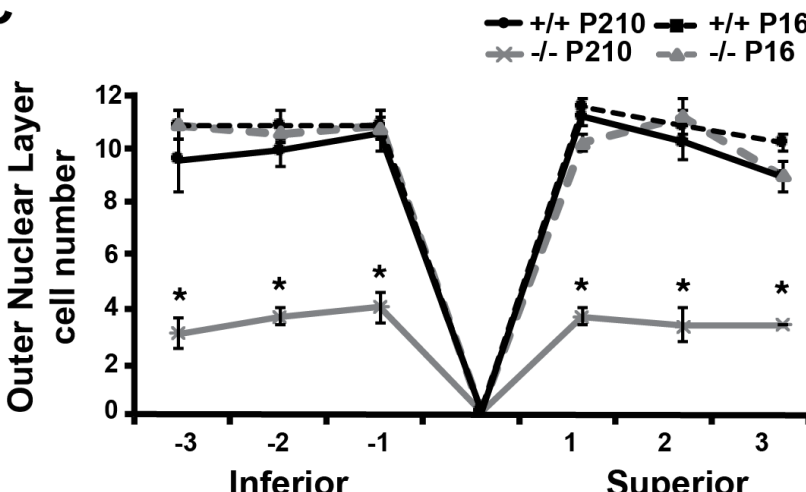

$\times-l-\mathrm{P} 210--1-\mathrm{P} 16$

\section{Figure 3. Degeneration of photoreceptor cells with loss of ARL2BP.}

(A and B) Retinal cross-sections of wildtype (+/+) and knockout (-/-) littermates stained with propidium iodide to demonstrate the loss of photoreceptor nuclei in the outer nuclear layer (ONL) at different ages (A, P16) and (B, P210). (C) Quantification of the ONL length (corresponding to the number of nuclei) between $+/+$ and $-/-$ littermates at different locations within the retina from the inferior ( -3 ) to superior (3) portion at P16 and P210. (scale bar $=20 \mu \mathrm{m}$ ). Data are represented as mean \pm SEM $\left(\mathrm{n}=3\right.$, unpaired two-tailed $\mathrm{t}$-test; $\left.{ }^{*} \mathrm{P}<0.01\right)$. $\mathrm{ONL}=$ outer nuclear layer; $\mathrm{INL}=$ inner nuclear layer. the animals aged

(Figure 2B). The ERG

reduction seen in $1-b p$

deletion animals was

analogous to the ERG

produced by those

with an 8-bp deletion.

The animals with a 6-

bp deletion that led to

an in-frame removal

of two amino acids

$(\Delta 16-17)$ displayed

normal retinal

function

(Supplemental Figure 2), and served as a control to demonstrate the specificity of the sgRNA used to generate the $\mathrm{KO}$ mice. Altogether, these results demonstrate the need for ARL2BP in photoreceptor function.

\section{ARL2BP is necessary for photoreceptor cell survival.}

The early loss in photoreceptor function suggested a possible defect in the development of photoreceptor cells. Therefore, we examined the retinal morphology at P16 by staining with 
the nuclear marker propidium iodide. Interestingly, at P16, when ERG responses are reduced, retinal lamination appears normal in the absence of ARL2BP. Additionally, we observed occasional brightly stained nuclei in the $\mathrm{KO}$ sections at $\mathrm{P} 16$, indicative of photoreceptor cell
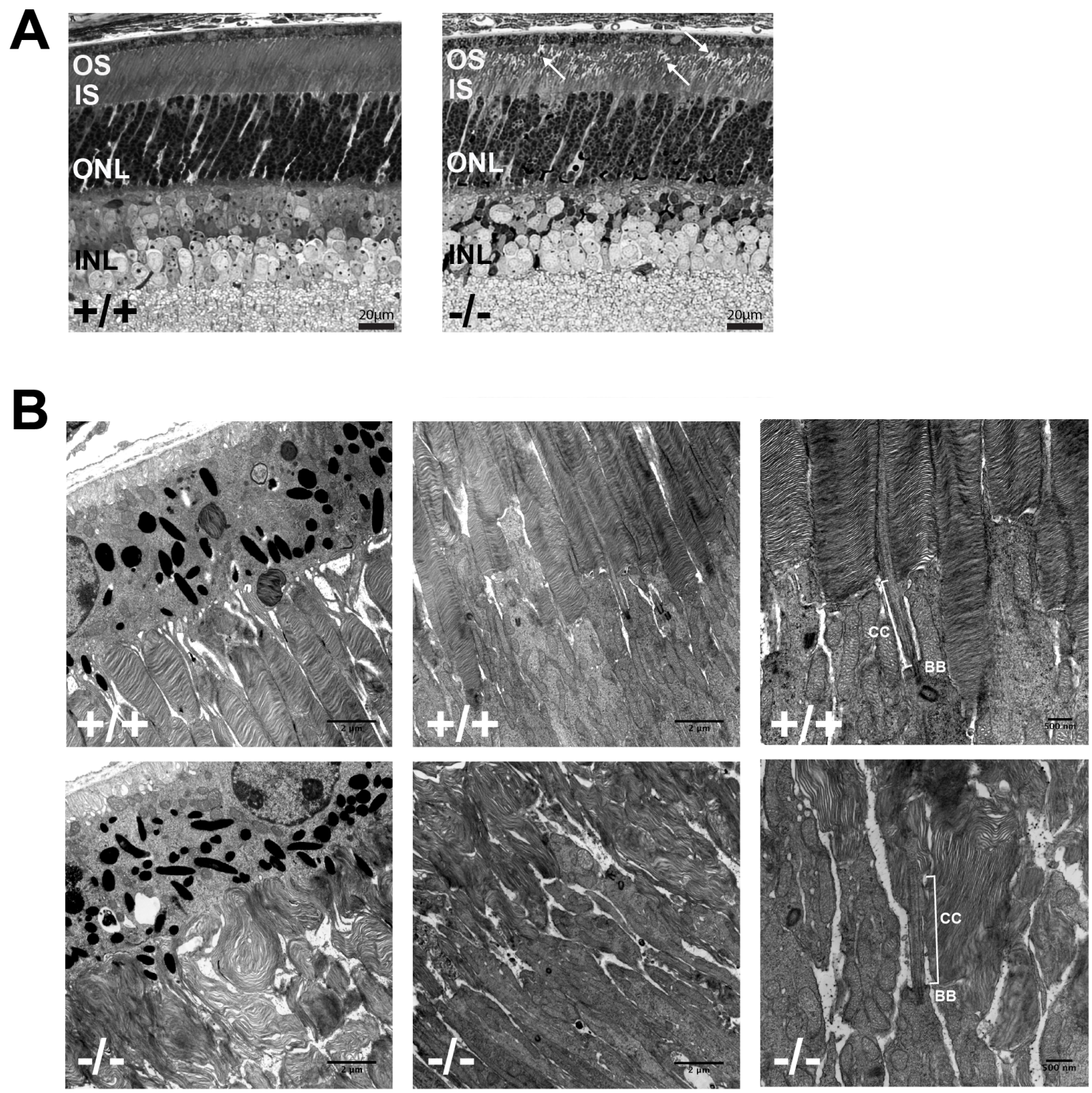

Figure 4. Abnormal photoreceptor outer segment disc morphology in the absence of ARL2BP

(A) Representative toluidine blue stained retinal cross-sections from wildtype $(+/+)$ and knockout (-/-) littermates at P16. Arrows point to disorganized OSs. (Scale bar $=20 \mu \mathrm{m}$ ). (B) Electron micrographs of retinal cross sections of $+/+$ and $-/$ - littermates at P16 illustrating the structural abnormalities of the OS in the KO. The left two panels of electron micrographs were taken at lower magnifications (scale bar $=2 \mu \mathrm{m}$ ); the right panel of electron micrographs were taken at a higher magnification $($ scale bar $=500 \mathrm{~nm}$ ). $\mathrm{ONL}=$ outer nuclear layer; $\mathrm{INL}=$ inner nuclear layer; $\mathrm{CC}=$ connecting cilium; $\mathrm{BB}=$ basal body.

death (Figure 3A). The development of inner neurons was normal, as there was no alteration in 
bipolar or horizontal cell numbers (Supplemental Figure 3).

However, at P210, the majority of photoreceptor nuclei were lost, signifying degeneration of photoreceptor cells in the absence of ARL2BP (Figure 3B, C and Supplemental Figure 4). This loss in photoreceptors supported the ERG response at this age (90\% reduction), and based on these findings, we conclude that ARL2BP is required for photoreceptor survival.

\section{Loss of ARL2BP induces altered photoreceptor morphology.}

We considered that the reduced photoreceptor function at P16 could be attributed to a disruption in the phototransduction cascade, either caused by a decrease in protein expression or a change in protein localization. Therefore, we first assessed the expression levels of known phototransduction and OS structural proteins. Immunoblot analysis of P16 retinal lysates revealed a trend for reduction of OS protein levels in ARL2BP KO photoreceptors, including

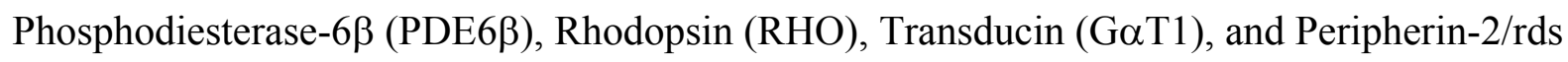
(PRPH-2) (Supplemental Figure 5). In contrast, expression levels of multiple inner segment proteins remained comparable between WT and KO (Supplemental Figure 5).

Next, we examined the localization of proteins needed for efficient phototransduction and/or OS morphogenesis. We analyzed retinal cross-sections at P16 of WT and KO animals (before photoreceptor degeneration) and found that the localization of multiple outer segment and inner segment proteins were maintained in KO photoreceptors, including PDE6 $\beta$, Rhodopsin, CNGA1, PRPH-2, and GaT1 (Supplemental Figure 6). 

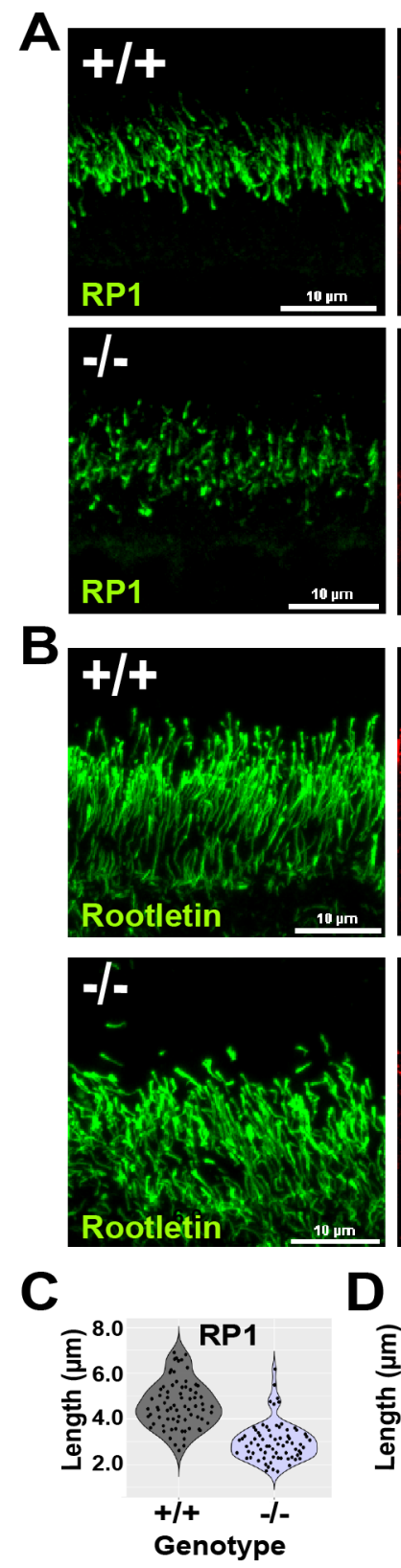
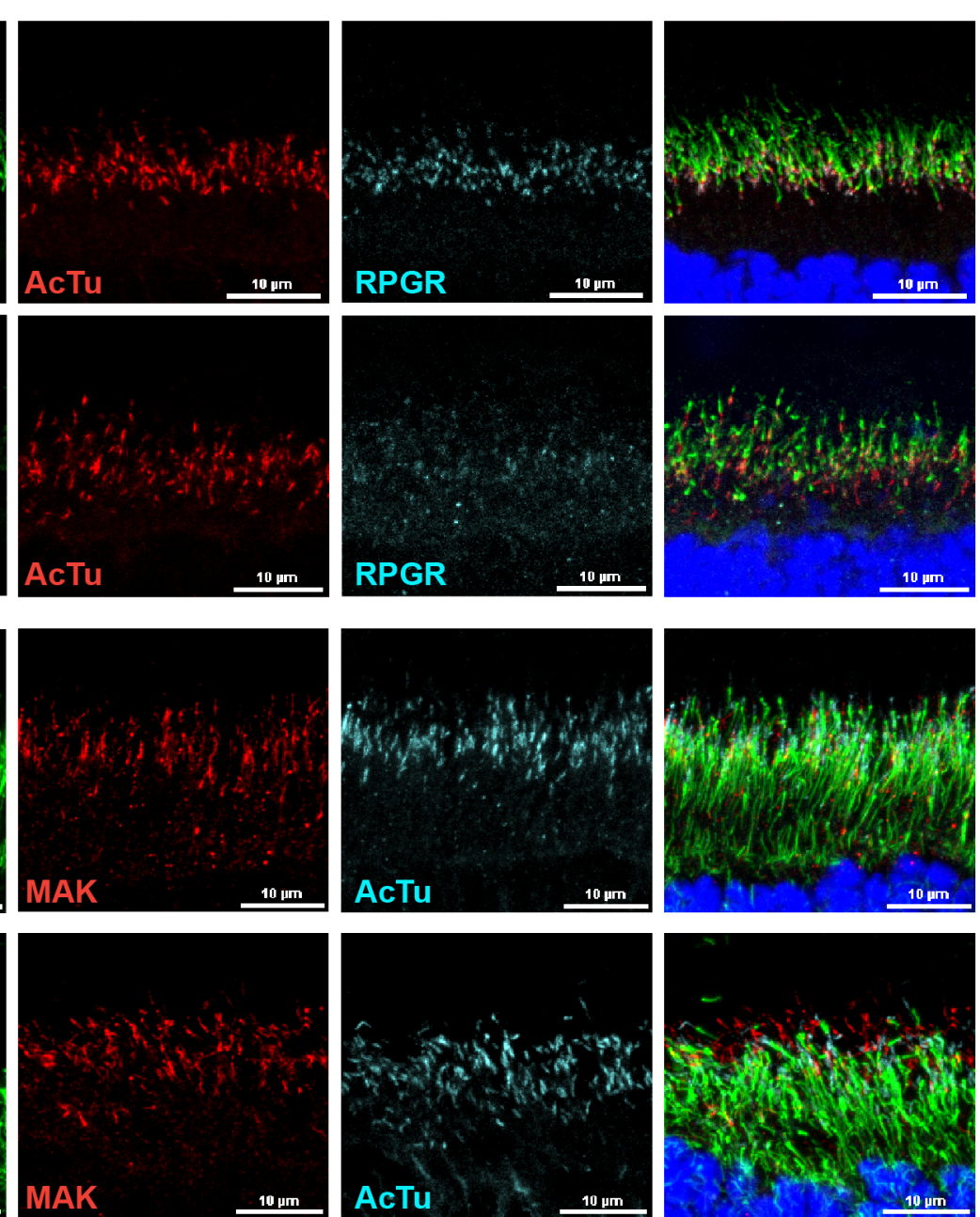

E
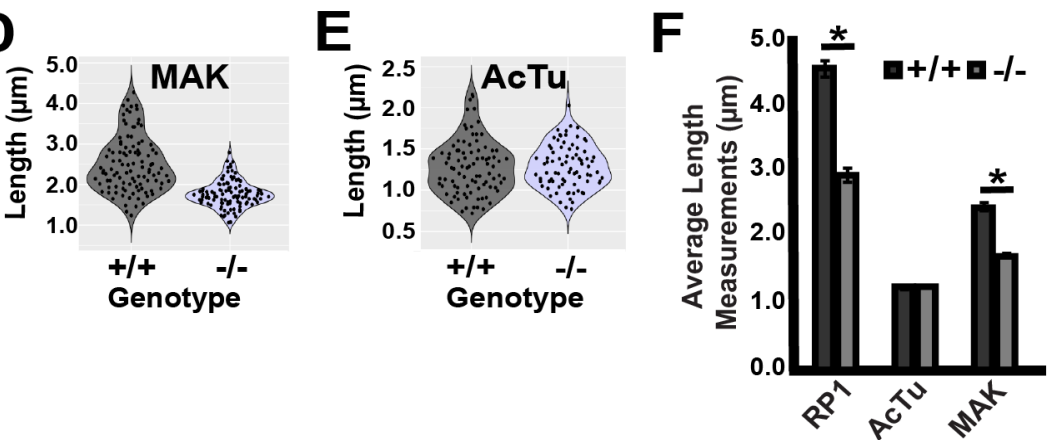

Figure 5. ARL2BP is needed for maintenance of photoreceptor axoneme.

(A and $B$ ) Retinal cross sections from wildtype (+/+) and knockout (-/-) littermates at P16 showing localization for (A) Retinitis Pigmentosa 1 (RP1, green), Acetylated $\triangle$-tubulin (AcTu, red), and Retinitis Pigmentosa GTPase regulator (RPGR, cyan) (scale bar $=10 \mu \mathrm{m}$ ) and (B) Rootletin (green), Male germ-cell associated kinase (MAK, red), and AcTu (cyan) (scale bar $=10 \mu \mathrm{m}$ ). (C-E) A frequency distribution illustrating the percentage of total measured axonemes. (C) length from tip of AcTu to tip of RP1, (D) length from tip of AcTu to tip of MAK, (E) length from base to tip of AcTu. (F) Quantitation of total measured axonemes and connecting cilia from P16 +/+ and -/-. Data are represented as mean \pm SEM $\left(n=300\right.$, unpaired two-tailed t-test; $\left.{ }^{*} P<0.05\right)$. 
There was a uniform reduction in phototransduction proteins and OS structural proteins at

P16, which we hypothesize is due to a combination of factors, including shortened OS,

photoreceptor cell death, and a consequent degradation of OS proteins. Moreover, since no one

OS protein was exclusively reduced, this indicates that phototransduction can still occur, albeit at

A
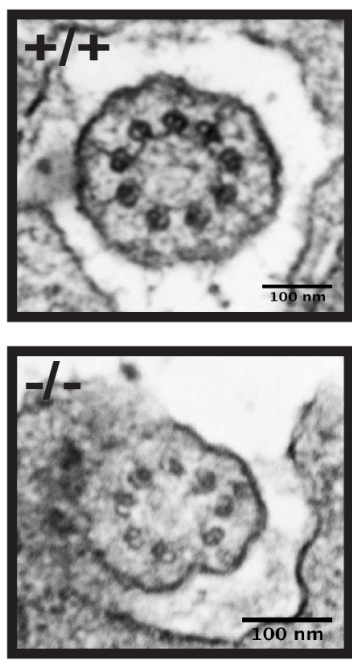

B
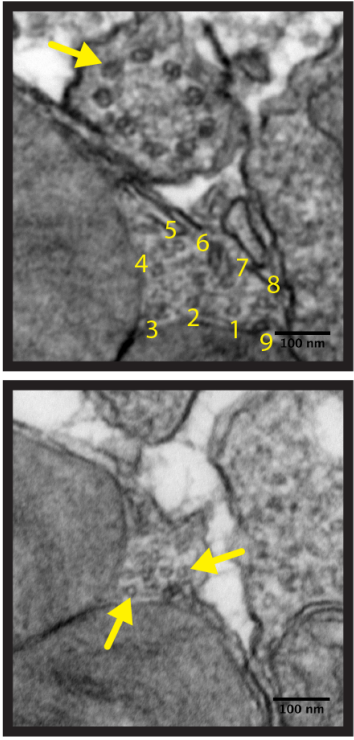
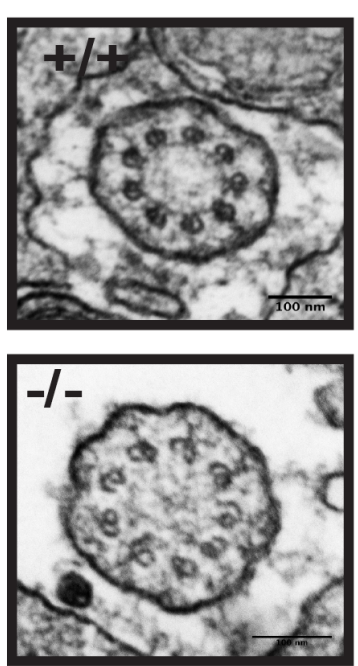
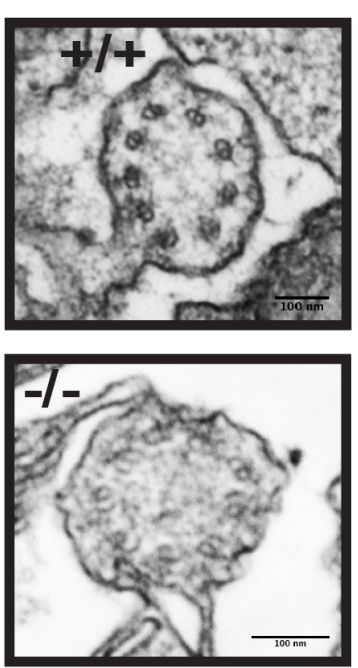
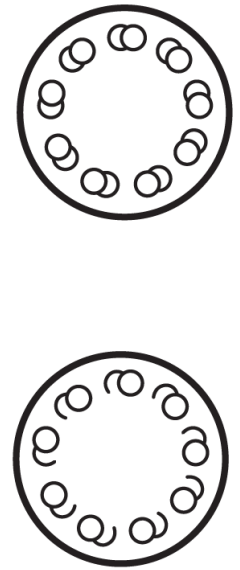

$-1-$
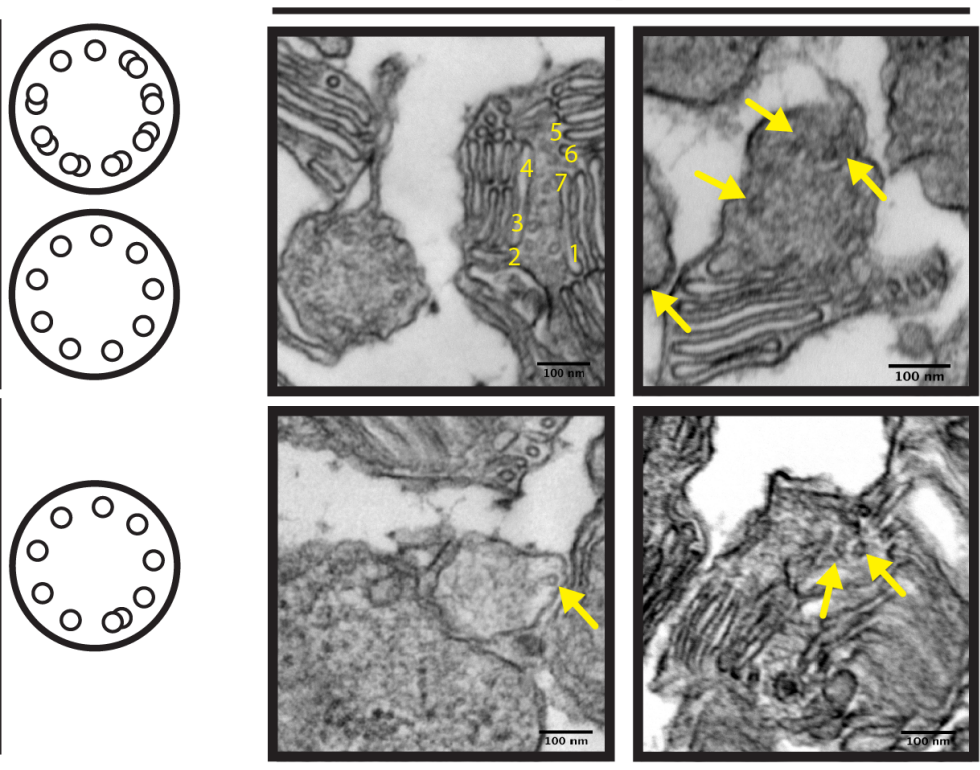
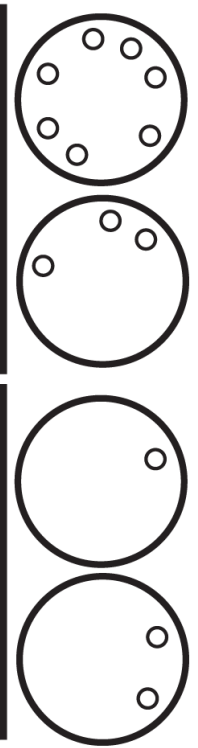

Figure 6. ARL2BP is required for normal doublet and singlet MT formation in photoreceptors.

(A and B) Cross-sectional transmission electron micrographs from wildtype $(+/+)$ and knockout $(-/-)$ retina at P16 illustrating the (A) structural defects in doublet microtubules of the $-/$-photoreceptor CC, displaying open B-tubules (scale bar $=100 \mathrm{~nm}$ ) and $(B)$ singlet and doublet microtubules present in more distal regions of the axoneme. +/+ (left panels) illustrates 9 singlet MTs in both sections shown, whereas -/- sections never displayed 9 singlets. Numbers and arrows refer to singlets (scale bar $=100 \mathrm{~nm}$ ). 
a reduced strength. This result is supported by the fact that WT and KO mice did not display a major difference in ERG sensitivity or in OS protein localization (Figure 2C and Supplemental Figure 6). Therefore, we hypothesized that ERG reduction was caused by an OS structural defect. This hypothesis was reinforced through light microscopy using toluidine blue stained retinal sections at P16, displaying distorted and ragged outer segments in the absence of ARL2BP (Figure 4A). To examine the OS structure in further detail, transmission electron microscopy (TEM) was performed on WT and KO eyecups at P17. In contrast to the wellorganized and precisely stacked OS discs seen in WT photoreceptors, KOs displayed dysmorphic OS, which included misoriented and irregular discs of variable diameters, fragmented OS, and membranous whorls (Figure 4B and C). This data demonstrates that loss of ARL2BP impairs photoreceptor disc morphogenesis and causes severe defects in OS structure.

\section{Shortened photoreceptor axonemes in the absence of ARL2BP.}

Due to the gross morphological defects in ARL2BP KO photoreceptors, and the presence of ARL2BP in the cilia, we hypothesized that ARL2BP loss could affect ciliary structure. TEM images from longitudinal sections displayed seemingly normal $\mathrm{CC}$ ultrastructure in $\mathrm{KO}$ retina; unfortunately, the thin sections used for this approach prevent population measurements of ciliary length. We therefore employed fluorescent microscopy, which has been widely used in the photoreceptor field for ensemble measurements of axoneme and CC lengths (Zhao et al., 2003; Liu et al., 2004a; Omori et al., 2010; Dilan et al., 2017). Using freshly frozen, unfixed eyecups from P16 WT and KO mice, the localization of multiple ciliary markers was investigated and the axonemes and $\mathrm{CC}$ were measured. In the absence of ARL2BP, the CC (transition zone) in photoreceptors forms properly, as demonstrated by acetylated tubulin (AcTu) staining (Figure 5A). This result was supported by $\mathrm{CC}$ length measurements from WT and KO 
TEM samples, which were found to be comparable $(1.25 \mu \mathrm{m} \pm 0.05, n=15$, Figure $4 \mathrm{C})$. However, widely-used photoreceptor axonemal markers such as RP1 and MAK, revealed a reduction in

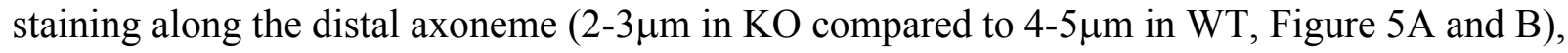
indicating a defect in the extension of the axoneme. One hundred ciliary measurements from three different mice are displayed in violin plots to show the distribution of lengths for RP1, MAK (axoneme), and AcTu (CC) (Figure 5C, D, and E, respectively), and were quantified in Figure $5 \mathrm{~F}$.

Intriguingly, while investigating the localization of multiple ciliary markers, we noticed that staining of Retinitis Pigmentosa GTPase Regulator (RPGR), a photoreceptor-specific ciliarelated protein (Meindl et al., 1996; Kirschner et al., 1999), was severely diminished in KO sections at P16 (Figure 5A). Overall, these results indicate a critical role for ARL2BP in the maintenance of photoreceptor axoneme and RPGR localization.

\section{ARL2BP loss disrupts doublet microtubule formation.}

Defects in axonemal extension in ARL2BP-null animals prompted us to assess crosssections of photoreceptor cilia structure with TEM. We confirmed the formation of $9+0$ doublet microtubules (DMTs) in photoreceptor CC of KO animals. However, most strikingly, animals lacking ARL2BP displayed abnormal DMT organization, with multiple microtubules possessing open B-tubules (Figure 6A). In contrast, open B-tubules were never observed in WT photoreceptor CC. This phenotype is reminiscent of the ARL13b Hennin mutant, the only other animal model reported to display this phenotype (Caspary et al., 2007). Upon examination of distal regions in the axoneme, we observed the expected change from doublet to singlet MTs in WT photoreceptors (Figure 6B). However, in the distal regions of KO photoreceptor axonemes we find a reduced number of singlet structures. Additionally, we were unable to identify a 9+0 
arrangement of microtubular structures (Figure 6B). This corroborates our findings from RP1 immunostaining, bearing in mind that RP1 is a photoreceptor-specific microtubule-associated protein which links the OS discs to the axoneme, predominantly in the distal region where singlets are present (Liu et al., 2004a) (Figure 5). In conclusion, ARL2BP KO photoreceptors are defective in inner junction B-tubule closure, consequently signifying a role for ARL2BP in photoreceptor DMT formation and axoneme extension. 


\section{Discussion}

In this study, we generated a global knockout of ARL2BP. This animal model phenocopied defects reported in patients with ARL2BP mutations, including the presentation of situs inversus (Davidson et al., 2013). However, the current study focused on the cilia-specific role for ARL2BP in photoreceptor cells. The absence of ARL2BP led to a progressive loss of function and degeneration of photoreceptors. We observed early morphological defects that include abnormal ciliary microtubule doublet structure with open B-tubules. We hypothesize that abnormal doublet structure likely leads to impaired extension of the photoreceptor distal axoneme and a consequent compromise of disc morphogenesis and OS structure.

\section{ARL2BP is necessary for photoreceptor function.}

Lack of ARL2BP leads to a decrease in photoreceptor function, even at an early age (P16). Most interestingly, OS disc morphology is affected with most discs aligned vertically rather than horizontally. We attribute the reduction in photoreceptor function to dysmorphic and shortened OS. This conclusion is supported by our findings that the sensitivity of photoresponse remains unaltered (Figure 2C), and results that indicate that the phototransduction cascade is intact (Supplemental Figure 5).

Due to the suggested role for ARL2BP in ciliogenesis and the importance of cilia in protein movement, we originally hypothesized that loss of ARL2BP would affect the trafficking of OS resident proteins. However, OS proteins appear to be properly localized in ARL2BP KO photoreceptors at P16, indicative of normal trafficking (Supplemental Figure 6). We did observe a slight mislocalization of rhodopsin (Supplemental Figure 6C), which we believe is due to the inability of the shortened OS to accommodate the massive rhodopsin protein 
levels normally present in the OS (Pearring et al., 2013). Based on these results, we conclude that OS protein trafficking is not the primary culprit of vision loss in the absence of ARL2BP.
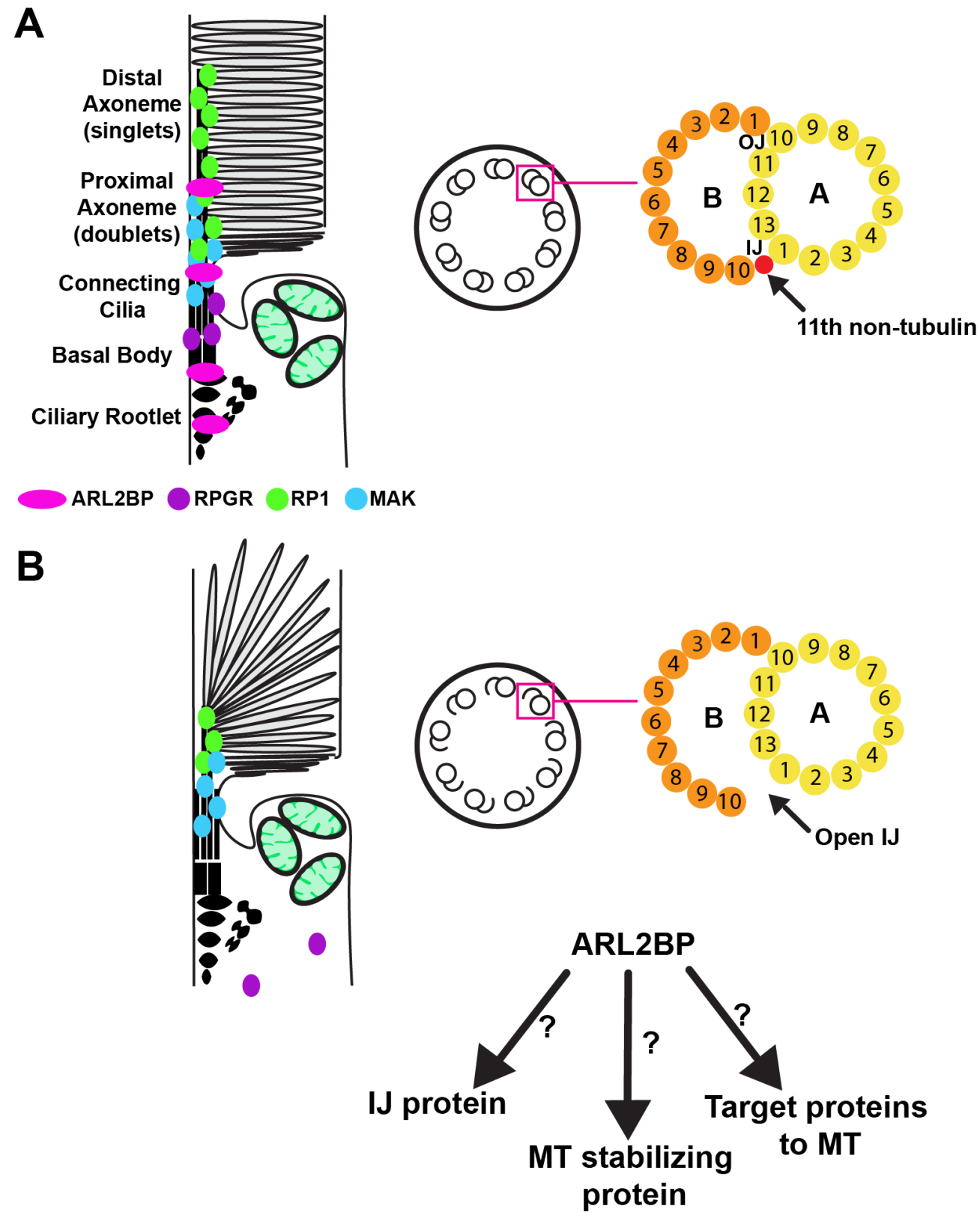

Figure 7. Role for ARL2BP in photoreceptors.

(A and B) Scheme illustrating the role of ARL2BP in photoreceptor microtubular axoneme extension, OS disc alignment, and ciliary protein localization. With ARL2BP (A), photoreceptor OS develops normally, with correct DMT formation, axoneme extension, and disc alignment. Without ARL2BP (B) axonemes are shorter, OS discs are not properly stacked, and DMT formation is disrupted indicating a role for ARL2BP in DMT formation and/or stability. IJ=Inner Junction; OJ=Outer Junction. 
Intriguingly, a few IS resident proteins were found to be mislocalized in ARL2BP KO retina. Specifically, Syntaxin 3 (STX3) and Receptor expression-enhancing protein 6 (REEP6) were found in the OS of ARL2BP KO retina at P16 (Supplemental Figure 7). This finding for STX3 has been previously shown in BBS mutant models (Datta et al., 2015; Dilan et al., 2017; Hsu et al., 2017), and thought to indicate a specific role for the BBSome as a gatekeeper in photoreceptor cells. However, ARL2BP does not interact with the BBSome, and absence of ARL2BP does not affect BBSome sub-unit protein expression (Supplemental Figure 7 and data not shown). Therefore, we propose that disturbances in the formation or stability of the photoreceptor cilia could also result in IS protein mislocalization.

\section{ARL2BP is indispensable for normal OS morphology.}

ARL2BP KO photoreceptors displayed highly disorganized OS, yet BBs and CC of seemingly normal ultrastructure in longitudinal sections. Importantly however, fluorescent microscopy analysis with axonemal markers revealed that axonemes were shorter with loss of ARL2BP at P16. A decrease in RP1 and MAK staining (axonemal markers) was also seen at P10 (Supplemental Figure 8). These results demonstrate a defect in photoreceptor axoneme extension during development of the OS. Additionally, it has been shown that horizontal disc stacking is disrupted in RP1 KO mouse models (Liu et al., 2003). Thus, it is possible that the vertical disc alignment seen in ARL2BP KO mice may be a direct consequence of the change in RP1, and perhaps other axonemal proteins, primarily caused by unstable or shortened axonemes.

Based on our results from ARL2BP KO animals, we envision three possible models for the role of ARL2BP in photoreceptors that we discuss below:

\section{ARL2BP loss results in ciliary protein mislocalization.}


RPGR is a ciliary transition zone protein that has a retina-specific isoform, mutations in which cause X-linked retinitis pigmentosa (Kirschner et al., 1999). Intriguingly, RPGR localization was severely affected with loss of ARL2BP. RPGR KO mouse models, however, cause a much milder phenotype than what is seen with loss of ARL2BP. It has also been shown that loss of RPGR in combination with the ablation of other ciliary proteins, such as CEP290 and RPGRIP, causes a much more severe defect than with loss of RPGR alone (Hong et al., 2001; Zhao et al., 2003; Anand and Khanna, 2012; Rao et al., 2016). Yet, we found both CEP290 and RPGRIP staining unaffected with loss of ARL2BP (Supplemental Figure 9). Therefore, we hypothesize that RPGR mislocalization is a secondary effect of ARL2BP loss, due to structural disruptions of the axoneme and does not contribute significantly to the phenotype of the ARL2BP knockout animals.

Alternatively, we cannot discount the possibility that RPGR and ARL2BP directly interact. However, our attempts to verify an interaction by immunoprecipitation with retinal lysates have failed, making this possibility unlikely.

\section{ARL2BP interaction with ARL2 GTPase affects microtubular axoneme assembly.}

ARL2BP, or BART, was originally named for its proposed interaction with the small GTPase ARL2 (ADP-ribosylation factor like 2) (Sharer and Kahn, 1999). Significantly, x-ray crystallography revealed a unique binding conformation between ARL2BP and ARL2-GTP (Zhang et al., 2009). However, our attempts to confirm this interaction in photoreceptors have so far been unproductive (Supplemental Figure 10). Nonetheless, ARL2's known interaction with tubulin and tubulin chaperones (Bhamidipati et al., 2000; Tian et al., 2010; Nithianantham et al., 2015; Francis et al., 2017), prompts a compelling direction of study for the role of ARL2BP in the development and stability of photoreceptor MTs. Therefore, more sensitive 
assays will be employed to examine their interaction and its importance in photoreceptors.

Impaired doublet microtubule assembly with loss of ARL2BP causes instability of axoneme.

Our most remarkable discovery concerns the photoreceptor DMT structure of the CC and axoneme, which was abnormal in the absence of ARL2BP. ARL2BP KO photoreceptor CC appear to have lost the inner junction closure, leaving an opening in the B-tubule (Figure 7B). This result is similar to the ARL13b Hennin KO which showed open B-tubules in the primary cilia of embryonic nodes (Caspary et al., 2007). Recent studies on the loss of ARL13b in retina have shown severe photoreceptor dysfunction with no photoresponse, accompanied by complete loss of OS (Hanke-Gogokhia et al., 2017; Dilan, et. al., 2018. In revision). Given that these models display a more severe phenotype than observed in our studies on ARL2BP, and that ARL13b protein expression and localization was comparable between ARL2BP WT and KO retina (Supplemental Figures 5 and 11), ARL2BP must be uniquely required for DMT formation in ciliary axonemes. It is possible that ARL2BP serves as a component of the inner junction protein complex (which is a non-tubulin protofilament (Nicastro et al., 2011; Pigino et al., 2012)) or that ARL2BP plays a role in stabilizing DMT structure as a microtubule associated protein (MAP). It is also possible that ARL2BP is involved in targeting microtubule interacting proteins (MIPs) to the DMTs, such as MIP3, a protein known to support the B-tubule structure (Nicastro et al., 2011; Ichikawa et al., 2017).

Furthermore, the loss of singlet MTs in the distal portions of KO photoreceptor axonemes indicate that improper DMT formation results in unstable ciliary extension (Figure 7). Correspondingly, it has been shown that transport along the DMT is A- and B-specific, where dynein motor proteins travel along the A-tubule and kinesin travels along the B-tubule (Stepanek and Pigino, 2016). Any disruption in the anterograde transport (B-tubule-specific) of axoneme 
building proteins consequently results in the inability of the axoneme to elongate (Prevo et al., 2017). Therefore, we hypothesize that the impairment in ciliary extension seen with loss of ARL2BP disrupts the ability of photoreceptors to form properly stacked OS discs (Scheme illustrated in Figure. 7A and B).

Our studies show the need for ARL2BP in DMT formation and core axoneme structure and elongation. The study of ARL2BP also highlights the importance of axoneme structure and stability in OS disc formation. How changes in the DMT and axoneme structure lead to altered photoreceptor outer segment organization and function will provide exciting insight into photoreceptor biology as well as ciliopathy-related fields. 


\section{Materials and Methods}

\section{Generation of Mouse Model and Genotyping}

The generation and cloning of the targeted sgRNA was performed as described in (Yang et al., 2013). Briefly, 20-nt sgRNA oligonucleotides (Supplemental Table 2) specific for a region in exon 2 of ARL2BP immediately upstream of the 5' - NGG PAM sequence were annealed and ligated into pX330 vector (pSpCas9). Upon DNA purification, the T7 promoter was added to the sgRNA template by PCR amplification (primer is Supplemental Table 2). Following gel extraction, the T7-sgRNA template was used for in vitro transcription using a kit from Clonetech. The sgRNA was then purified using an RNA purification kit from Ambion. The specificity and efficacy of the sgRNA/Cas9 cutting was confirmed by in vitro assays. Both the sgRNA and Cas9 mRNA (Invitrogen) were injected into the pronuclei of FVB blastocysts (17 $\mathrm{ng} / \mathrm{ul}$ and $34 \mathrm{ng} / \mathrm{ul}$, respectively). The correctly targeted founders were identified by sequencing of the genomic DNA fragment surrounding the ARL2BP exon 2 region (Supplemental Table 2). The mice were then backcrossed into C57black 6/J to remove the $r d 1$ allele naturally present in FVB mice. However, multiple backcrosses into the C57black 6/J strain led to concomitant reduction in number of knockout animals obtained. Therefore, mice were crossed into 129/SV-E mice (Strain \# 476 from Charles River Laboratories), which somewhat restored the expected Mendelian ratios of knockout animals obtained from heterozygous crosses. Due to the extensive backcrossing (8 total rounds), we could rule out "off-target" effects sometimes associated with Crispr-Cas9 gene editing. Genotyping was performed using primers listed in Supplemental Table 2 on ear punch samples and the PCR fragments were analyzed by capillary electrophoresis or by 7\% polyacrylamide/Urea gels followed by detection with the LI-COR odyssey system. 
Institutional Animal Care and Use Committee of the West Virginia University approved all experimental procedures involving animals conducted in this study.

\section{Generation of Antibody against full-length ARL2BP}

ARL2BP antibody was generated as previously described (Wright et al., 2016). Briefly, ARL2BP with a C-terminal his-tag was expressed in Origami E.coli strain (Novagen). Origami cells were grown to an $\mathrm{OD}_{600} \approx 0.7$ and protein production was induced with $0.5 \mathrm{mM}$ IPTG for 18 hours at $16^{\circ} \mathrm{C}$. The protein was purified from the soluble fraction using a Nickel His Affinity Column and the eluate was supplied to Pacific Immunology Corp. for generation of the antibody in rabbits. Antibody serum was then purified against a column cross-linked with GST-ARL2BP fusion protein and tested via immunoblotting in both HEK 293 cells expressing ARL2BP and retinal extracts.

\section{Immunoblotting}

Mice were euthanized by $\mathrm{CO}_{2}$ inhalation followed by cervical dislocation and eyes were enucleated. Following dissection, flash frozen retinas were sonicated in phosphate buffered saline [PBS: 137mM NaCl, $2.7 \mathrm{mM} \mathrm{KCl}, 4.3 \mathrm{mM} \mathrm{Na}_{2} \mathrm{HPO}_{4} \cdot 7 \mathrm{H}_{2} \mathrm{O}, 1.4 \mathrm{mM} \mathrm{K \textrm {KH } _ { 2 }} \mathrm{PO}_{4}$, with protease inhibitor cocktail (Roche)] and protein concentrations were measured using a Nano Drop spectrophotometer (Thermo Fisher Scientific, Inc.). Equal amounts of samples (100 $\mu \mathrm{g}$ total protein per well) were separated on a polyacrylamide SDS-PAGE and transferred onto polyvinylidene difluoride membranes (Immunobilon-FL, Millipore, Billerica). Membranes were blocked with western blot blocking buffer (Rockland Inc.) for $30 \mathrm{~min}$ at room temperature and incubated with primary antibodies overnight at $4^{\circ} \mathrm{C}$ (antibody dilutions in Supplemental Table 1). Blots were washed in PBST (PBS with 0.1\% Tween-20) 3 times for 5 min (3 X 5 min) at room temperature and incubated in secondary antibody, goat anti-rabbit Alexa 680, goat anti-rat Alexa 
680, or goat anti-mouse Alexa 800 (Invitrogen) for $30 \mathrm{~min}$ at room temperature. After 3 x $5 \mathrm{~min}$ of washes with PBST, membranes were scanned using Odyssey Infrared Imaging System (LICOR Biosciences, Lincoln, NE, USA).

\section{Immunofluorescence}

Enucleated eyes were immersed in 4\% paraformaldehyde (PFA) fixative for 15 min prior to removal of the cornea and lens. Eyecups were fixed for a total of $2 \mathrm{hr}$ and washed in 1X PBS for $20 \mathrm{~min}$, followed by incubation in $20 \%$ sucrose/PBS overnight at $4^{\circ} \mathrm{C}$. Afterward, eyes were incubated in a 1:1 mixture of 20\% sucrose in PBS and OCT (Cryo Optimal Cutting Temperature Compound, Sakura) for $1 \mathrm{hr}$, and flash frozen in OCT.

To stain specifically for cilia in photoreceptor cells, eyes were fixed for $30 \mathrm{sec}$ in $4 \%$ PFA after removal of anterior segments and washed in 1X PBS for $1 \mathrm{~min}$. They were then immediately flash frozen in OCT.

Cryosectioning was performed with a Leica CM1850 Cryostat, and serial retinal sections of $16 \mu \mathrm{m}$ or $12 \mu \mathrm{m}$ (for cilia) thickness were mounted on Superfrost Plus slides (Fisher Scientific). Retinal sections mounted on slides were washed with 1X PBS and incubated for $1 \mathrm{hr}$ in blocking buffer (PBS with 5\% goat sera, $0.5 \%$ TritonX-100, 0.05\% sodium azide). Retinal sections were then incubated with primary antibody overnight at $4^{\circ} \mathrm{C}$ (dilutions defined in Supplemental Table 1), followed by two 15 min washes with PBSTx (PBS with $0.1 \%$ TritonX100), and one with PBS $1 \mathrm{x}$ before incubation with secondary antibody at 1:1000 dilution for $1 \mathrm{hr}$. After three 15 min washes with PBSTx, the sections were mounted with ProLong Gold antifade reagent (Life Technologies) and viewed on a Nikon Eclipse Ti C2 confocal microscope using excitation wavelengths of 405, 488, 543, and $647 \mathrm{~nm}$. 
For propidium iodide staining, retinal sections were incubated with propidium iodide for $1 \mathrm{hr}$ at RT following blocking. They were then washed three times for 15 min with PBSTx.

\section{Transmission Electron Microscopy (TEM)}

Prior to enucleation, transcardial perfusions were performed on the mice for $10 \mathrm{~min}$ using $15 \mathrm{mls}$ of $2 \%$ Paraformaldehyde, $2 \%$ Glutaraldehyde, and $0.05 \% \mathrm{CaCl}_{2}$, in $50 \mathrm{mM}$ MOPS buffer. Once completed, the cornea and lens were removed from enucleated eyes and kept in fixative for $1 \mathrm{hr}$. Dissection, embedding, and TEM was completed according to previously published methods (Ku et al., 2015).

\section{Electroretinogram recording (ERG)}

Mice were dark-adapted overnight prior to testing. Mice were anesthetized $(2.0 \%$ isoflourane with 2.5 liters per minute (lpm) oxygen flow rate) for $10 \mathrm{~min}$. Test subject eyes were topically dilated with a 1:1 mixture of tropicamide: phenylephrine hydrochloride. Mice were placed on a heated platform with a continuous flow of isoflourane anesthesia through a nose cone (1.5\% isoflourane with $2.5 \mathrm{lpm}$ oxygen flow rate). A reference electrode was placed subcutaneously in the scalp and ERG responses were recorded from both eyes with silver wire electrodes placed above each cornea, with contact being made with a drop of hypromellose solution (2\% hypromellose in PBS) (Gonioscopic Prism Solution, Wilson Ophthalmic, Mustang, OK, USA). Rod-dominated responses (scotopic) were elicited in the dark with flashes of LED white light at increasing flash intensities. Light-adapted cone responses (photopic) were elicited with white light flashes in the presence of a $30 \mathrm{~cd} / \mathrm{m} 2$ rod-saturating white background light. ERGs were performed on the UTAS Visual Diagnostic System with BigShot Ganzfeld with UBA-4200 amplifier and interface, and EMWIN 9.0.0 software (LKC Technologies, Gaithersburg, MD, USA). 


\section{Subretinal Injections}

Plasmid DNA was injected and electroporated into the subretinal space of P0 mice according to a previously published protocol (Venkatesh et al., 2013). DNA solutions were prepared at $5 \mu \mathrm{g} / \mu \mathrm{l}$. Each animal received $0.5 \mu \mathrm{l}$ of DNA in the right eye, and no injection in the untreated contralateral eye.

\section{Statistical Analysis}

All data are presented as mean \pm standard error margin. ERG data and immunoblots (Supplemental) were analyzed by unpaired, two-tailed $t$ test $(n=3$ or 4 ). For cilia measurements, 80-100 cilia were measured for each animal $(n=3)$ and data were visualized with the ggplot2 package in $\mathrm{R}$ version 3.3.2. Image and densitometry analysis were performed using Image J $1.50 \mathrm{i}$. 


\section{Acknowledgements}

The authors thank Dr. Peter Stoilov, Dr. Zachary Wright (West Virginia University), and Dr. Tamara Caspary (Emory University) for help with data collection and analysis, Jessica Cunningham (West Virginia University) for help with transcardial perfusions, the Transgenic

Core for help with generation of the ARL2BP KO mouse model using the Crispr-Cas9 system (West Virginia University), and Paolo Fagone (West Virginia University Protein Core) for help with the purification of ARL2BP protein. This work was supported by National Institutes of Health Grants R01 EY028035 (to VR), R01 EY025536 (to VR and Peter Stoilov), R21 EY027707 (to VR), EY025291(to AFXG), and West Virginia Lions and Lions Club International Foundation. 


\section{References}

Anand, M., and Khanna, H. (2012). Ciliary transition zone (TZ) proteins RPGR and CEP290: role in photoreceptor cilia and degenerative diseases. Expert Opin Ther Targets 16, 541-551.

Bhamidipati, A., Lewis, S.A., and Cowan, N.J. (2000). ADP ribosylation factor-like protein 2 (Ar12) regulates the interaction of tubulin-folding cofactor D with native tubulin. J Cell Biol 149, 1087-1096.

Boldt, K., Mans, D.A., Won, J., van Reeuwijk, J., Vogt, A., Kinkl, N., Letteboer, S.J., Hicks, W.L., Hurd, R.E., Naggert, J.K., Texier, Y., den Hollander, A.I., Koenekoop, R.K., Bennett, J., Cremers, F.P., Gloeckner, C.J., Nishina, P.M., Roepman, R., and Ueffing, M. (2011). Disruption of intraflagellar protein transport in photoreceptor cilia causes Leber congenital amaurosis in humans and mice. J Clin Invest 121, 2169-2180.

Brown, P., Gibbons, I., and Wald, G. (1963). The visual cells and visual pigment of the mudpuppy, Necturus. J Cell Biol 19, 79-106.

Bujakowska, K.M., Liu, Q., and Pierce, E.A. (2017). Photoreceptor Cilia and Retinal Ciliopathies. Cold Spring Harb Perspect Biol 9.

Caspary, T., Larkins, C.E., and Anderson, K.V. (2007). The graded response to Sonic Hedgehog depends on cilia architecture. Dev Cell 12, 767-778.

Datta, P., Allamargot, C., Hudson, J.S., Andersen, E.K., Bhattarai, S., Drack, A.V., Sheffield, V.C., and Seo, S. (2015). Accumulation of non-outer segment proteins in the outer segment underlies photoreceptor degeneration in Bardet-Biedl syndrome. Proceedings of the National Academy of Sciences of the United States of America 112, E4400-E4409.

Davidson, A.E., Schwarz, N., Zelinger, L., Stern-Schneider, G., Shoemark, A., Spitzbarth, B., Gross, M., Laxer, U., Sosna, J., Sergouniotis, P.I., Waseem, N.H., Wilson, R., Kahn, R.A., Plagnol, V., Wolfrum, U., Banin, E., Hardcastle, A.J., Cheetham, M.E., Sharon, D., and Webster, A.R. (2013). Mutations in ARL2BP, encoding ADP-ribosylation-factor-like 2 binding protein, cause autosomal-recessive retinitis pigmentosa. Am J Hum Genet 93, 321-329.

Dilan, T.L., Singh, R.K., Saravanan, T., Moye, A., Goldberg, A.F.X., Stoilov, P., and Ramamurthy, V. (2017). Bardet-Biedl Syndrome -8 (BBS8) protein is crucial for the development of outer segments in photoreceptor neurons. Human Molecular Genetics, ddx399ddx399.

Francis, J.W., Goswami, D., Novick, S.J., Pascal, B.D., Weikum, E.R., Ortlund, E.A., Griffin, P.R., and Kahn, R.A. (2017). Nucleotide Binding to ARL2 in the TBCD·ARL2· $\beta$-Tubulin Complex Drives Conformational Changes in $\beta$-Tubulin. Journal of Molecular Biology.

Goldberg, A.F.X., Moritz, O.L., and Williams, D.S. (2016). Molecular basis for photoreceptor outer segment architecture. Progress in retinal and eye research in press. 
Hanke-Gogokhia, C., Wu, Z., Sharif, A., Yazigi, H., Frederick, J.M., and Baehr, W. (2017). The guanine nucleotide exchange factor, Arf-like protein 13b, is essential for assembly of the mouse photoreceptor transition zone and outer segment. Journal of Biological Chemistry.

Hong, D.H., Yue, G., Adamian, M., and Li, T. (2001). Retinitis pigmentosa GTPase regulator (RPGRr)-interacting protein is stably associated with the photoreceptor ciliary axoneme and anchors RPGR to the connecting cilium. J Biol Chem 276, 12091-12099.

Hsu, Y., Garrison, J.E., Kim, G., Schmitz, A.R., Searby, C.C., Zhang, Q., Datta, P., Nishimura, D.Y., Seo, S., and Sheffield, V.C. (2017). BBSome function is required for both the morphogenesis and maintenance of the photoreceptor outer segment. PLOS Genetics 13, e1007057.

Ichikawa, M., Liu, D., Kastritis, P.L., Basu, K., Hsu, T.C., Yang, S., and Bui, K.H. (2017). Subnanometre-resolution structure of the doublet microtubule reveals new classes of microtubule-associated proteins. Nat Commun $8,15035$.

Insinna, C., Pathak, N., Perkins, B., Drummond, I., and Besharse, J.C. (2008). The homodimeric kinesin, Kif17, is essential for vertebrate photoreceptor sensory outer segment development. Developmental biology 316, 160-170.

Kirschner, R., Rosenberg, T., Schultz-Heienbrok, R., Lenzner, S., Feil, S., Roepman, R., Cremers, F.P., Ropers, H.H., and Berger, W. (1999). RPGR transcription studies in mouse and human tissues reveal a retina-specific isoform that is disrupted in a patient with X-linked retinitis pigmentosa. Human molecular genetics $8,1571-1578$.

Knabe, W., and Kuhn, H. (1997). Ciliogenesis in photoreceptor cells of the tree shrew retina. Anat Embryol (Berl) 196, 123-131.

Ku, C.A., Chiodo, V.A., Boye, S.L., Hayes, A., Goldberg, A.F., Hauswirth, W.W., and Ramamurthy, V. (2015). Viral-mediated vision rescue of a novel AIPL1 cone-rod dystrophy model. Hum Mol Genet 24, 670-684.

Linck, R., Fu, X., Lin, J., Ouch, C., Schefter, A., Steffen, W., Warren, P., and Nicastro, D. (2014). Insights into the structure and function of ciliary and flagellar doublet microtubules: tektins, Ca2+-binding proteins, and stable protofilaments. J Biol Chem 289, 17427-17444.

Linck, R.W., and Stephens, R.E. (2007). Functional protofilament numbering of ciliary, flagellar, and centriolar microtubules. Cell Motil Cytoskeleton 64, 489-495.

Liu, Q., Lyubarsky, A., Skalet, J.H., Pugh, E.N., and Pierce, E.A. (2003). RP1 Is Required for the Correct Stacking of Outer Segment Discs. Investigative ophthalmology \& visual science 44, 4171-4183.

Liu, Q., Zuo, J., and Pierce, E.A. (2004a). The Retinitis Pigmentosa 1 Protein Is a Photoreceptor Microtubule-Associated Protein. The Journal of neuroscience : the official journal of the Society for Neuroscience 24, 6427-6436. 
Liu, Q., Zuo, J., and Pierce, E.A. (2004b). The retinitis pigmentosa 1 protein is a photoreceptor microtubule-associated protein. J Neurosci 24, 6427-6436.

May-Simera, H., Nagel-Wolfrum, K., and Wolfrum, U. (2017). Cilia - The sensory antennae in the eye. Prog Retin Eye Res 60, 144-180.

Meindl, A., Dry, K., Herrmann, K., Manson, E., Ciccodicola, A., Edgar, A., Carvalho, M.R.S., Achatz, H., Hellebrand, H., Lennon, A., Migliaccio, C., Porter, K., Zrenner, E., Bird, A., Jay, M., Lorenz, B., Wittwer, B., D'Urso, M., Meitinger, T., and Wright, A. (1996). A gene (RPGR) with homology to the RCC1 guanine nucleotide exchange factor is mutated in $\mathrm{X}$-linked retinitis pigmentosa (RP3). Nature Genetics 13, 35.

Molday, R.S., and Moritz, O.L. (2015). Photoreceptors at a glance. Journal of Cell Science 128, 4039-4045.

Nicastro, D., Fu, X., Heuser, T., Tso, A., Porter, M.E., and Linck, R.W. (2011). Cryo-electron tomography reveals conserved features of doublet microtubules in flagella. Proceedings of the National Academy of Sciences of the United States of America 108, E845-E853.

Nithianantham, S., Le, S., Seto, E., Jia, W., Leary, J., Corbett, K.D., Moore, J.K., and AlBassam, J. (2015). Tubulin cofactors and Arl2 are cage-like chaperones that regulate the soluble alphabeta-tubulin pool for microtubule dynamics. Elife 4, 1-33.

Omori, Y., Chaya, T., Katoh, K., Kajimura, N., Sato, S., Muraoka, K., Ueno, S., Koyasu, T., Kondo, M., and Furukawa, T. (2010). Negative regulation of ciliary length by ciliary male germ cell-associated kinase (Mak) is required for retinal photoreceptor survival. Proc Natl Acad Sci U S A 107, 22671-22676.

Pearring, J.N., Salinas, R.Y., Baker, S.A., and Arshavsky, V.Y. (2013). Protein sorting, targeting and trafficking in photoreceptor cells. Prog Retin Eye Res 36, 24-51.

Pierce, E.A., Quinn, T., Meehan, T., McGee, T.L., Berson, E.L., and Dryja, T.P. (1999). Mutations in a gene encoding a new oxygen-regulated photoreceptor protein cause dominant retinitis pigmentosa. Nat Genet 22, 248-254.

Pigino, G., Maheshwari, A., Bui, K.H., Shingyoji, C., Kamimura, S., and Ishikawa, T. (2012). Comparative structural analysis of eukaryotic flagella and cilia from Chlamydomonas, Tetrahymena, and sea urchins. Journal of Structural Biology 178, 199-206.

Pinto, L.H., Invergo, B., Shimomura, K., Takahashi, J.S., and Troy, J.B. (2007). Interpretation of the mouse electroretinogram. Documenta ophthalmologica. Advances in ophthalmology 115, 127-136.

Prevo, B., Scholey, J.M., and Peterman, E.J.G. (2017). Intraflagellar transport: mechanisms of motor action, cooperation, and cargo delivery. The FEBS Journal 284, 2905-2931.

Ramamurthy, V., and Cayouette, M. (2009). Development and disease of the photoreceptor cilium. Clinical Genetics 76, 137-145. 
Rao, K.N., Zhang, W., Li, L., Ronquillo, C., Baehr, W., and Khanna, H. (2016). Ciliopathyassociated protein CEP290 modifies the severity of retinal degeneration due to loss of RPGR. Hum Mol Genet 25, 2005-2012.

Sharer, J.D., and Kahn, R.A. (1999). The ARF-like 2 (ARL2)-binding protein, BART. Purification, cloning, and initial characterization. J Biol Chem 274, 27553-27561.

Steinberg, R., and Wood, I. (1975). Clefts and microtubules of photoreceptor outer segments in the retina of the domestic cat. J Ultrastructural Res 51, 397-403.

Stepanek, L., and Pigino, G. (2016). Microtubule doublets are double-track railways for intraflagellar transport trains. Science 352, 721.

Tian, G., Thomas, S., and Cowan, N.J. (2010). Effect of TBCD and its regulatory interactor Arl2 on tubulin and microtubule integrity. Cytoskeleton (Hoboken) 67, 706-714.

Venkatesh, A., Ma, S., Langellotto, F., Gao, G., and Punzo, C. (2013). Retinal gene delivery by rAAV and DNA electroporation. Curr Protoc Microbiol Chapter 14, Unit 14D 14.

Wensel, T.G., Zhang, Z., Anastassov, I.A., Gilliam, J.C., He, F., Schmid, M.F., and Robichaux, M.A. (2016). Structural and Molecular Bases of Rod Photoreceptor Morphogenesis and Disease. Progress in retinal and eye research 55, 32-51.

Yang, H., Wang, H., Shivalila, C.S., Cheng, A.W., Shi, L., and Jaenisch, R. (2013). One-step generation of mice carrying reporter and conditional alleles by CRISPR/Cas-mediated genome engineering. Cell 154, 1370-1379.

Young, R.W. (1967). The renewal of photoreceptor cells outer segments The Journal of Cell Biology 33, 61-72.

Zhang, T., Li, S., Zhang, Y., Zhong, C., Lai, Z., and Ding, J. (2009). Crystal structure of the ARL2-GTP-BART complex reveals a novel recognition and binding mode of small GTPase with effector. Structure 17, 602-610.

Zhao, Y., Hong, D.-H., Pawlyk, B., Yue, G., Adamian, M., Grynberg, M., Godzik, A., and Li, T. (2003). The retinitis pigmentosa GTPase regulator (RPGR)- interacting protein: Subserving RPGR function and participating in disk morphogenesis. Proceedings of the National Academy of Sciences of the United States of America 100, 3965-3970.

Zhou, C., Cunningham, L., Marcus, A.I., Li, Y., and Kahn, R.A. (2006). Arl2 and Arl3 regulate different microtubule-dependent processes. Mol Biol Cell 17, 2476-2487. 


\section{Supplemental Figures}
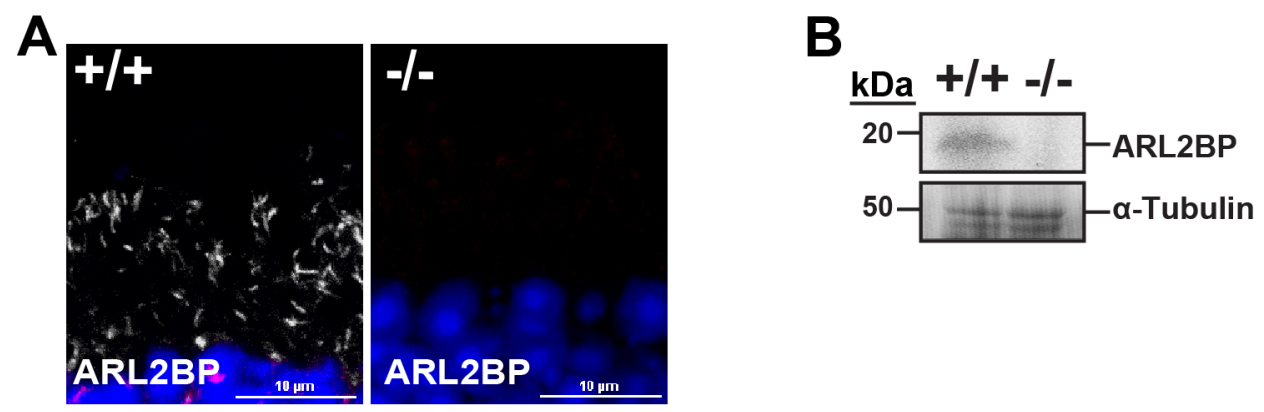

C
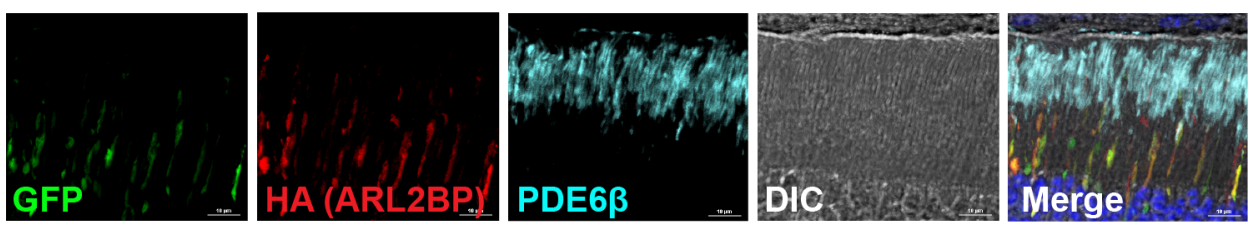

Supplemental Figure 1. ARL2BP localization in photoreceptor cells.

Panels (A and B) demonstrate the specificity of monoclonal antibody for ARL2BP between wildtype (+/+) and knockout (-/-) littermates in (A) retinal cross-sections and in B) retinal lysates. (C) Retinal cross section of CD-1 mice injected with plasmid expressing Hemagglutinin (HA)- tagged ARL2BP under chicken $\beta$-actin promoter (CAG), demonstrating ARL2BP exclusion from the outer segment. GFP is internal plasmid expression control. (GFP - green, HA - red (ARL2BP), PDE6 $\beta$ - cyan, nucleus - DAPI). PDE6 $\beta$ serves as the outer segment marker. $($ scale bar $=10 \mu \mathrm{m})$. 


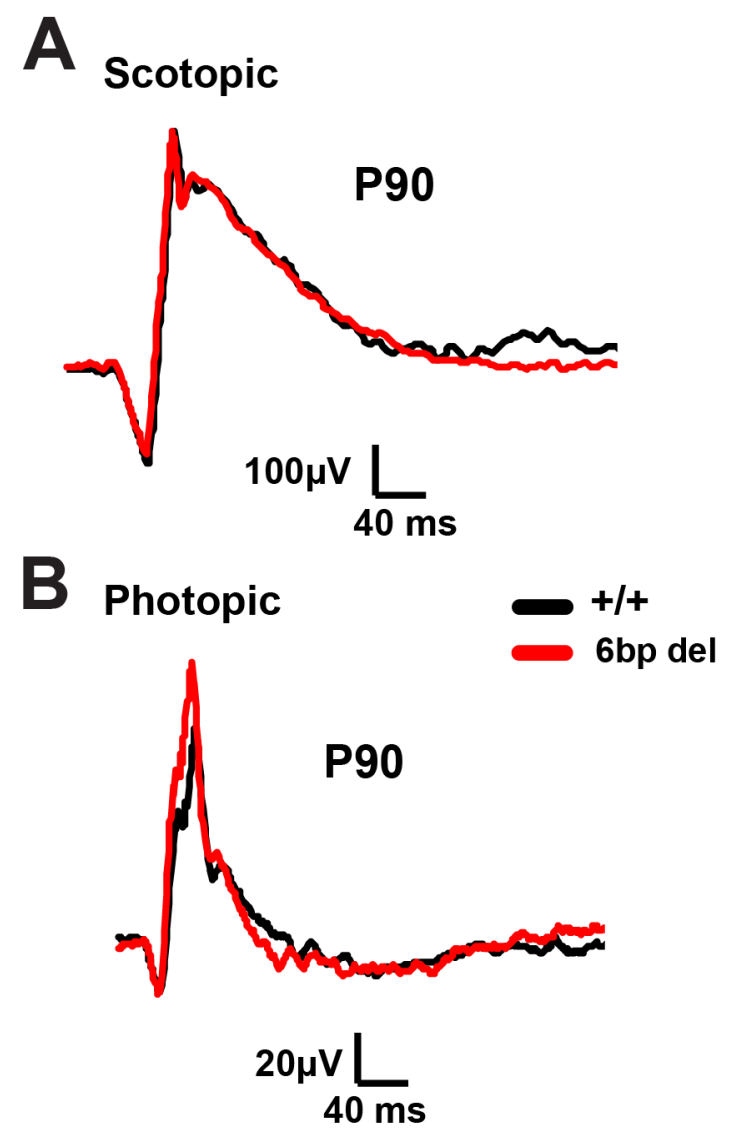

Supplemental Figure 2. Normal photoreceptor function in animals with ARL2BP 6bp deletion mutation.

(A and B) Representative scotopic (rod, A) and photopic (cone, B) electroretinograms (ERGs) at $-0.8 \mathrm{~cd} . \mathrm{s} \mathrm{m}^{-2}(\mathrm{~A})$ and $0.69 \mathrm{~cd} . \mathrm{s} \mathrm{m}^{-2}$ (B) comparing wildtype (+/+) and $6 \mathrm{bp}$ del animals at $\mathrm{P} 90$. 


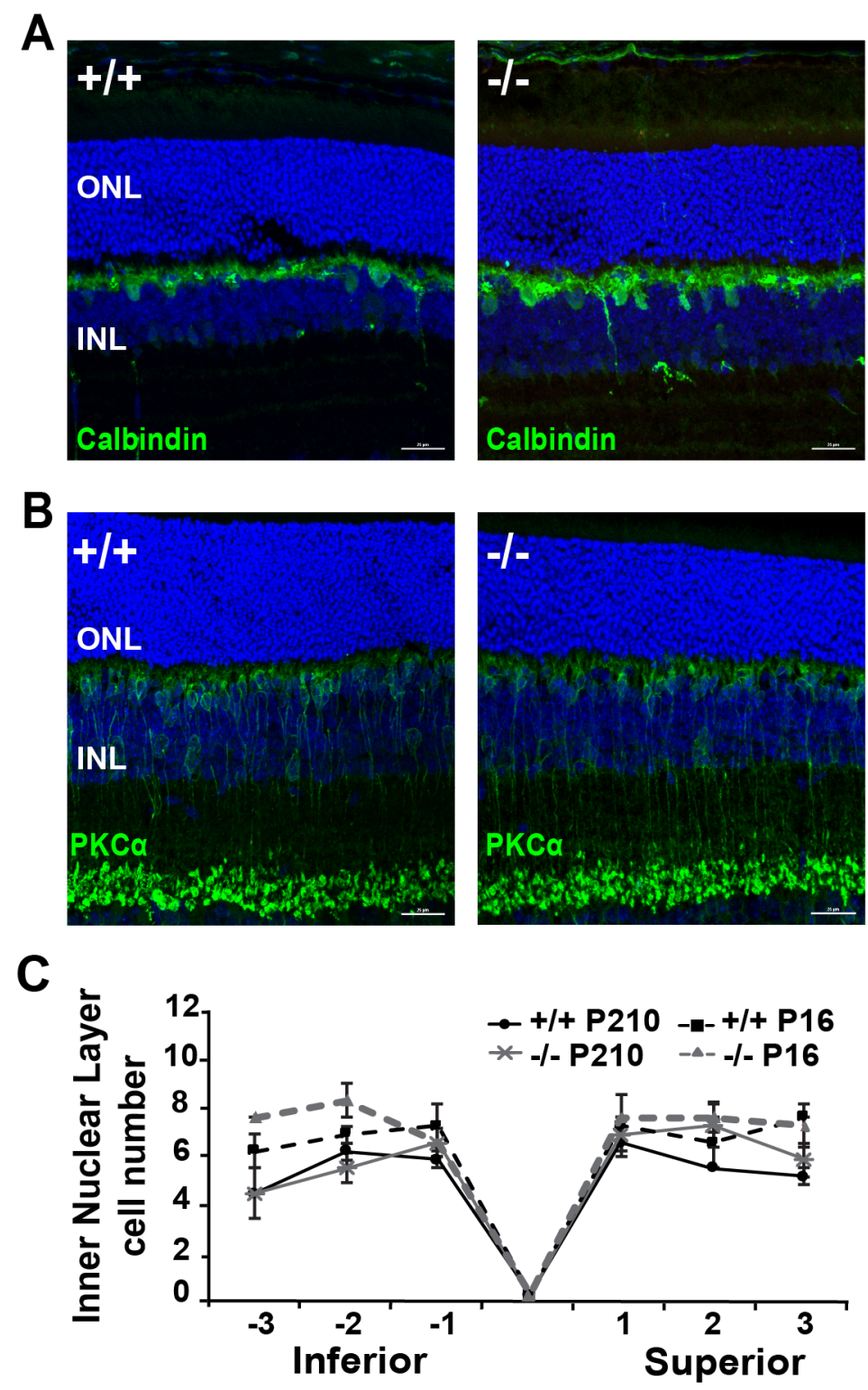

Supplemental Figure 3. Inner retinal cell layers unaffected by loss of ARL2BP. (A and B) Retinal sections from wildtype (+/+) and knockout (-/-) P16 littermates stained with the indicated inner retinal markers (Calbindin stains horizontal cells; PKC $\alpha$ stains bipolar cells). (Scale bar $=20 \mu \mathrm{m})$. (C) Quantification of the INL length (corresponding to the number of nuclei) between $+/+$ and -/- littermates at different locations within the retina from the inferior (3 ) to superior (3) portion at P16 and P210. Data are represented as mean $\pm \mathrm{SEM}(n=3$, unpaired two-tailed $t$-test). INL $=$ inner nuclear layer. $\mathrm{ONL}=$ outer nuclear layer. 

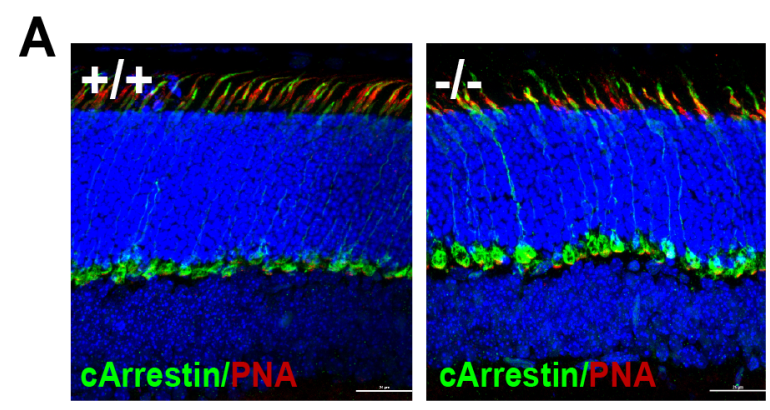

B
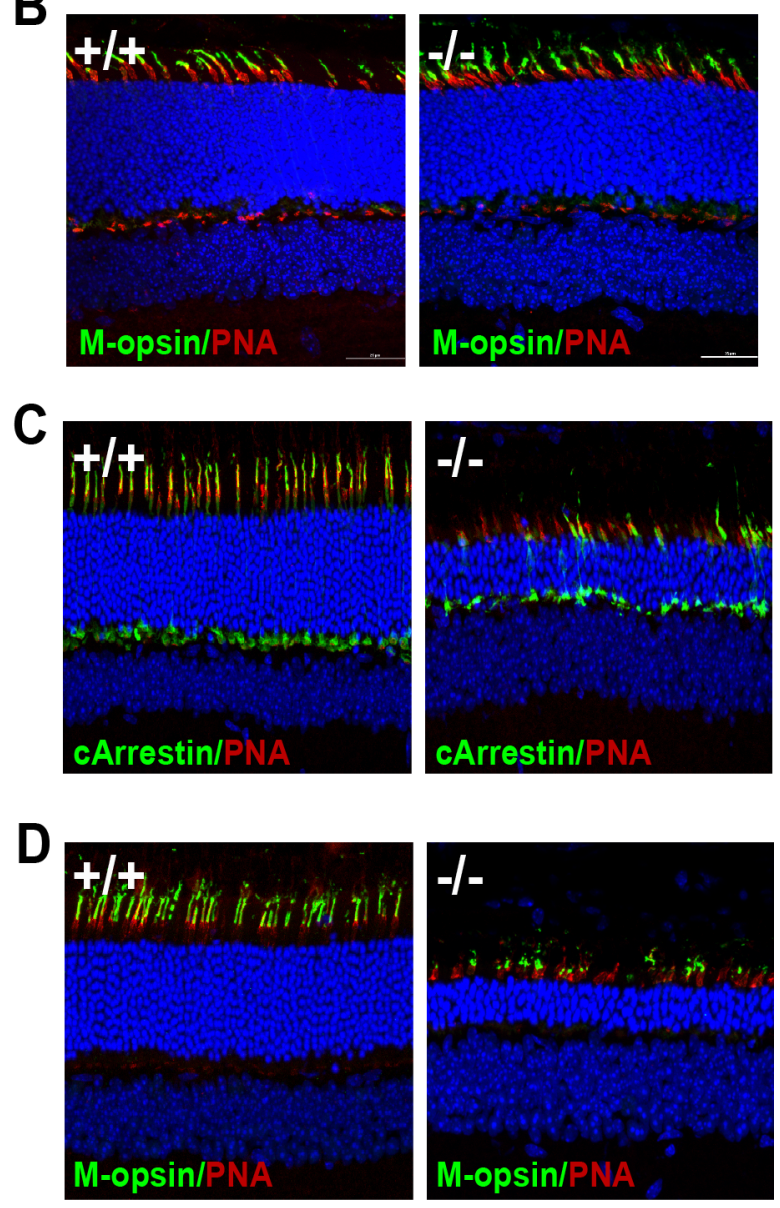

Supplemental Figure 4. Progressive loss of cones with loss of ARL2BP.

(A - D) Retinal sections from ARL2BP knockout (-/-) and littermate controls stained with indicated cone markers at P16 (A and B) and P210 (C and D). (scale bar $=20 \mu \mathrm{m})$. PNA $=$ peanut agglutinin. 

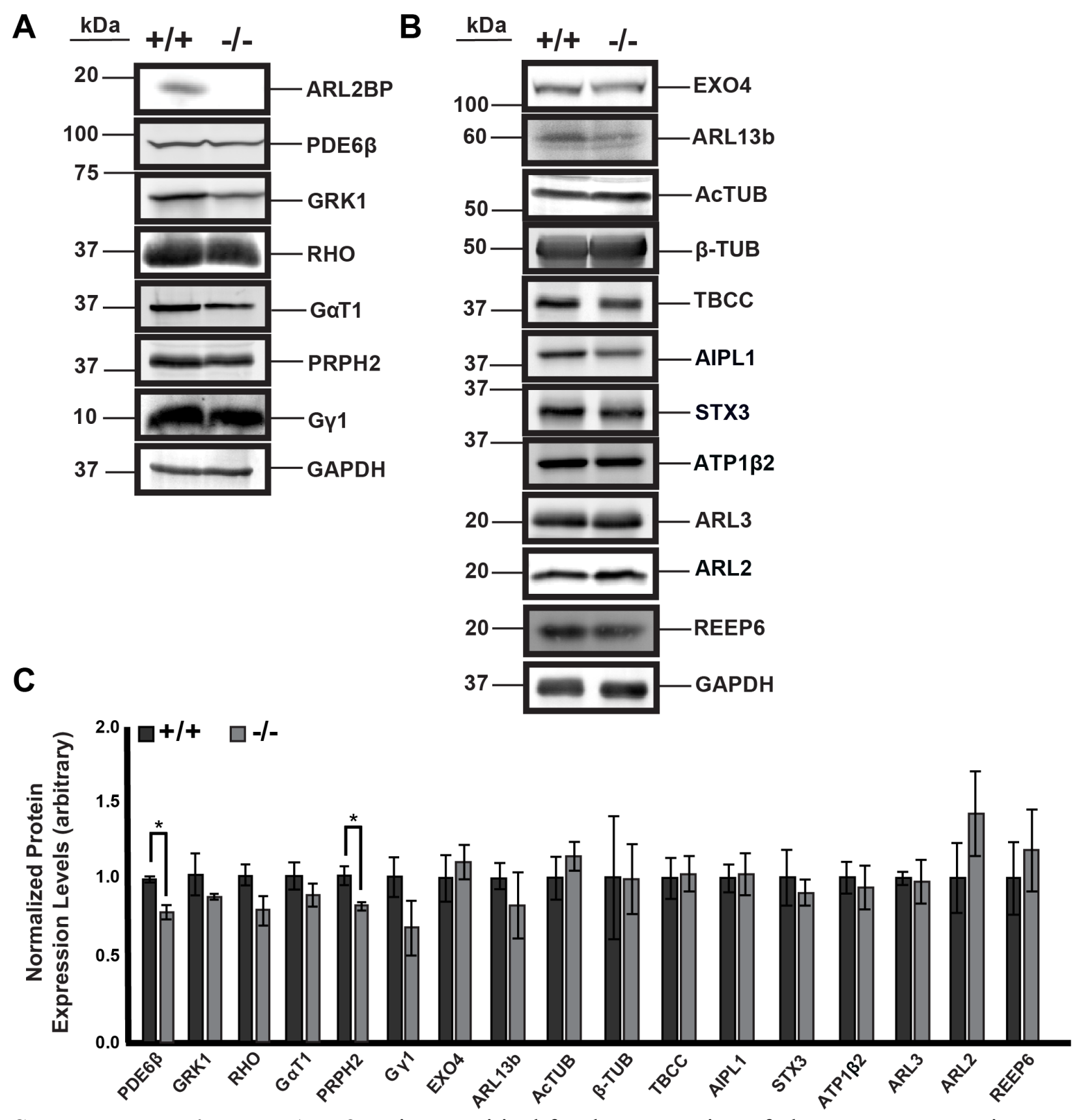

Supplemental Figure 5. ARL2BP is not critical for the expression of photoreceptor proteins. (A and B) Representative immunoblots displaying expression of proteins from retinal lysates of wildtype $(+/+)$ and knockout (-/-) littermates at P16. Protein molecular weight in kilodaltons $(\mathrm{kDa})$ is indicated on the left. (C) Quantification of protein levels from panels (A and B). Phosphodiesterase-6 $\beta$ (PDE6 $\beta$ ) and peripherin-2/rds (PRPH2) showed significant decrease in knockout animals compared to controls. All samples were normalized to GAPDH. Data are represented as mean $\pm \operatorname{SEM}(n=3$, unpaired two-tailed $t$-test; $* \mathrm{P}<0.05)$. 

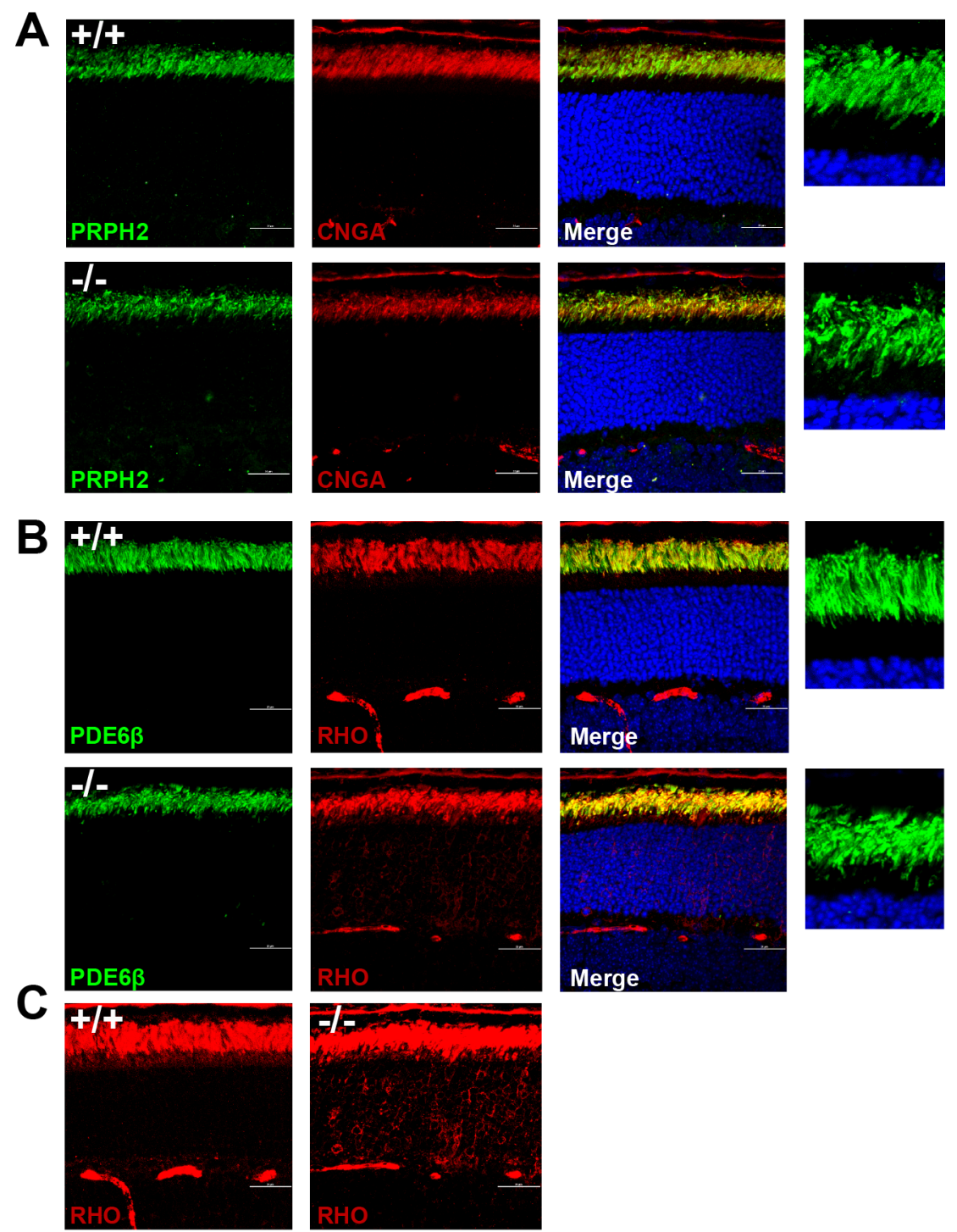

Supplemental Figure 6. Localization of outer segment proteins in animals lacking ARL2BP. (A and B) Retinal sections from P16 wildtype (+/+) and knockout (-/-) littermates stained with indicated outer segment markers. Zoomed images located in the far-right panel further illustrate localization of proteins in the OS. (C) Overexposed images from panel (B) to demonstrate mislocalization of Rhodopsin in -/.. (Scale bar $=20 \mu \mathrm{m})$. 


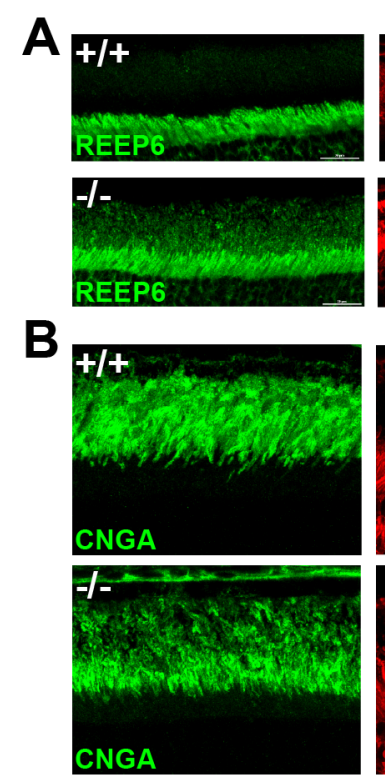

C

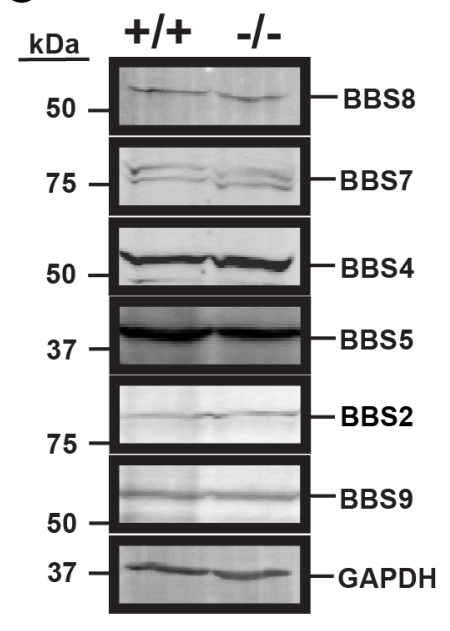

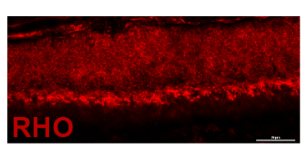
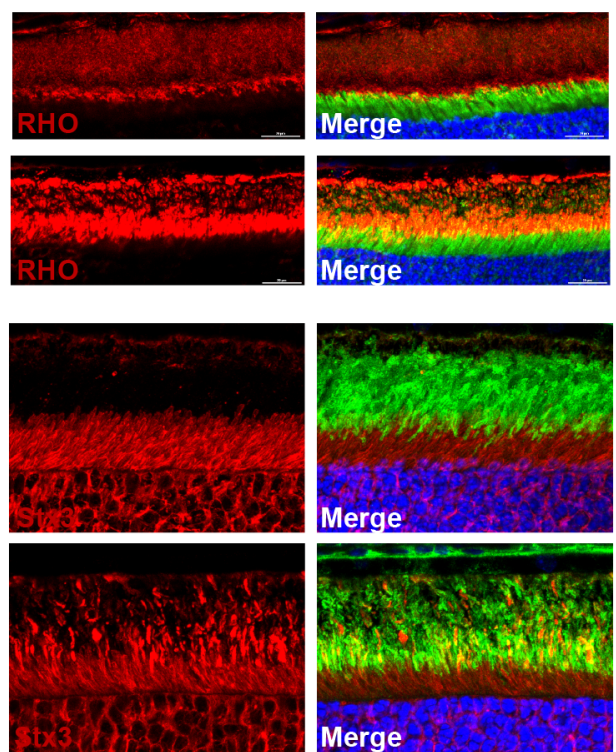

D

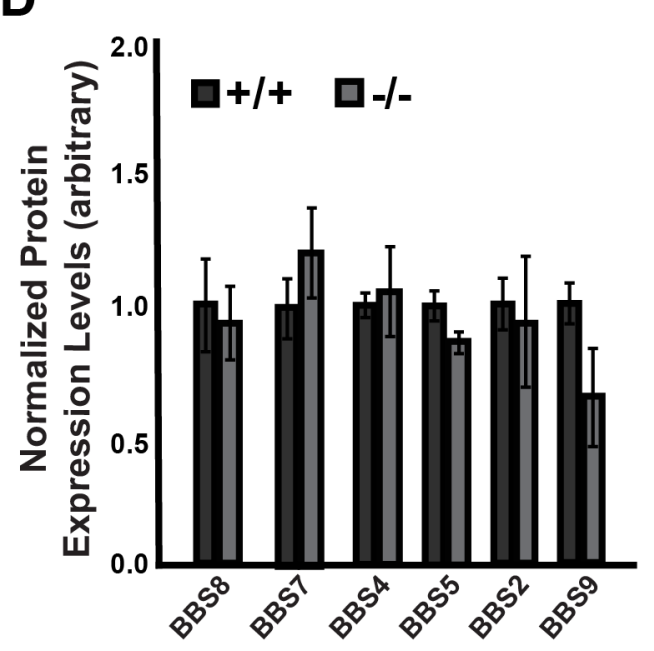

Supplemental Figure 7. Mislocalization of IS proteins in the absence of ARL2BP.

(A and B) Retinal sections from P16 wildtype (+/+) and knockout (-/-) littermates showing localization of normally IS-resident proteins (A) Syntaxin 3 (STX3, red) and (B) (REEP6, green) with OS proteins (A) cyclic nucleotide-gated channel (CNGA, green) and (B) Rhodopsin (RHO, red). (Scale Bar $=20 \mu \mathrm{m})$. (C) Representative immunoblots displaying expression of BBSome proteins from retinal lysates of wildtype $(+/+)$ and knockout $(-/-)$ littermates at P16. Protein molecular weight in kilodaltons $(\mathrm{kDa})$ is indicated on the left. (D) Quantification of protein levels from panel (C). 
A
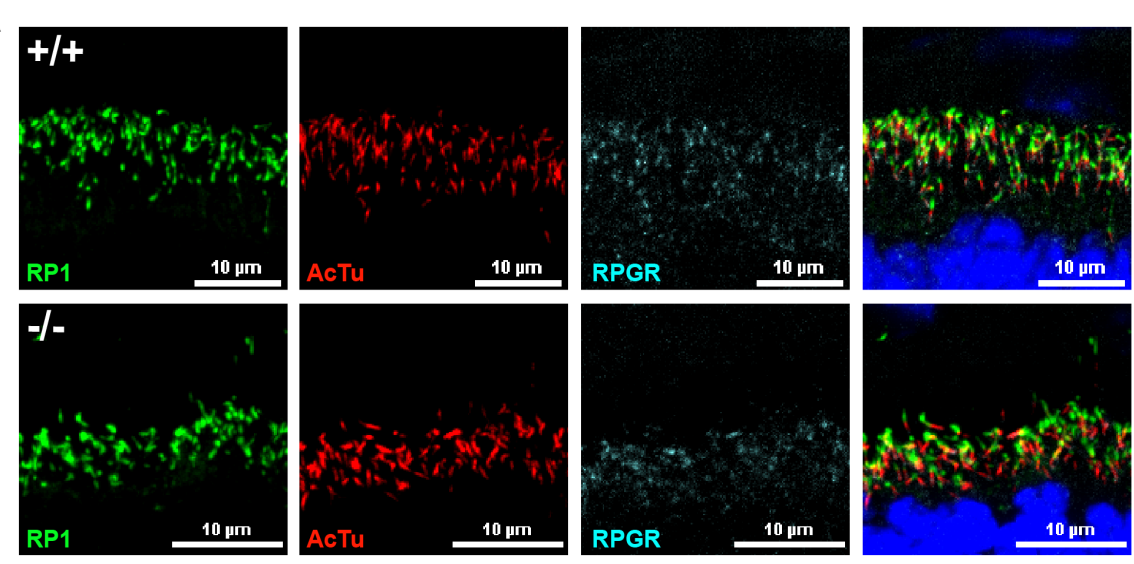

B
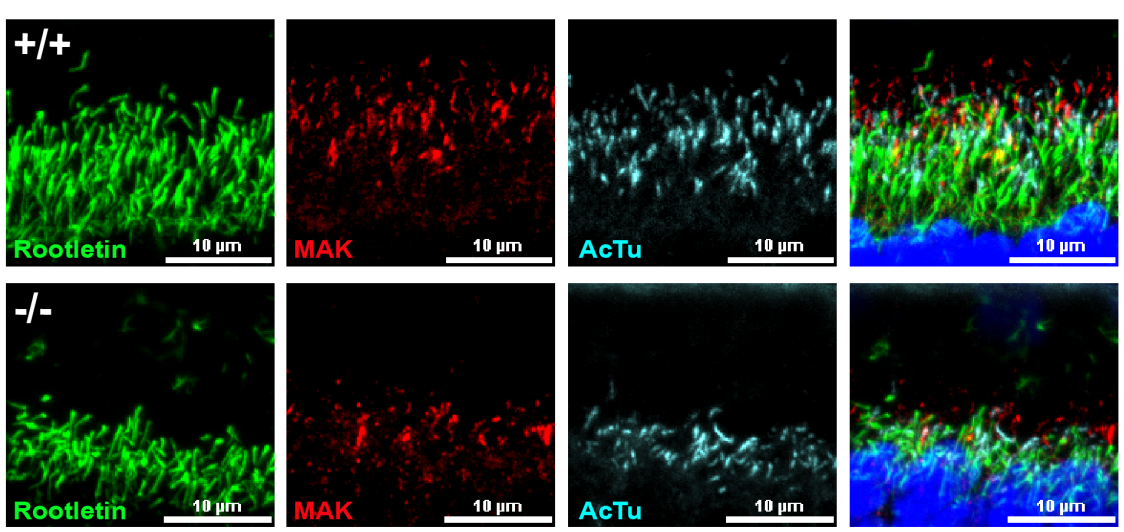

C

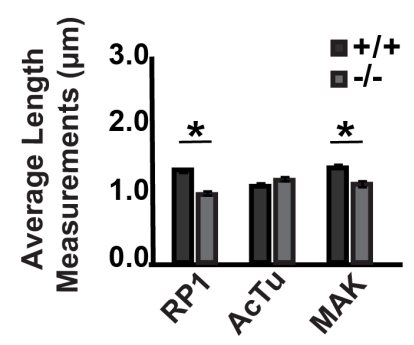

Supplemental Figure 8. Early reduction in photoreceptor axoneme lengths in the absence of ARL2BP.

(A and B). Retinal sections from wildtype (+/+) and knockout (-/-) littermates at P10 showing localization of (A) RP1 (green), AcTu (red), and RPGR (cyan), and (B) Rootletin (green), MAK (red), and AcTu (cyan). (scale bar $=10 \mu \mathrm{m}$ ). (C) Quantitation of total measured axonemes and 
connecting cilia from $\mathrm{P} 10+/+$ and $-/-$. Data are represented as mean \pm SEM $(n=300$, unpaired two-tailed $t$-test; $* \mathrm{P}<0.05$ ).
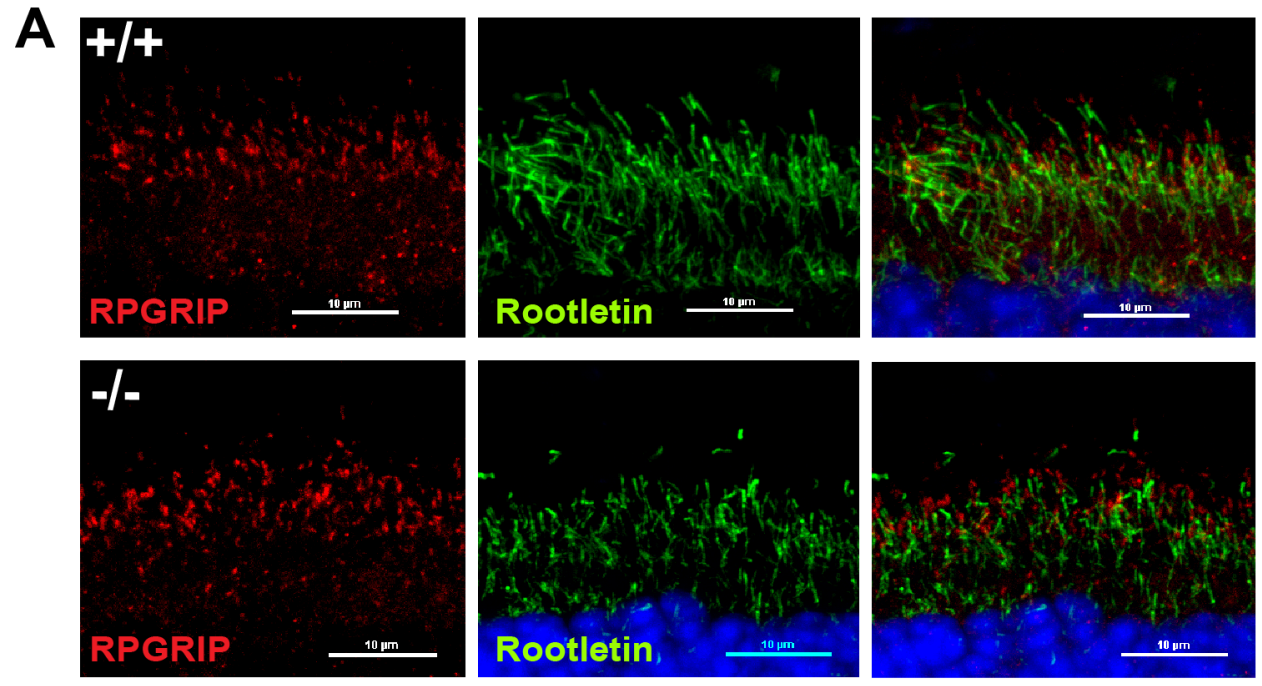

B
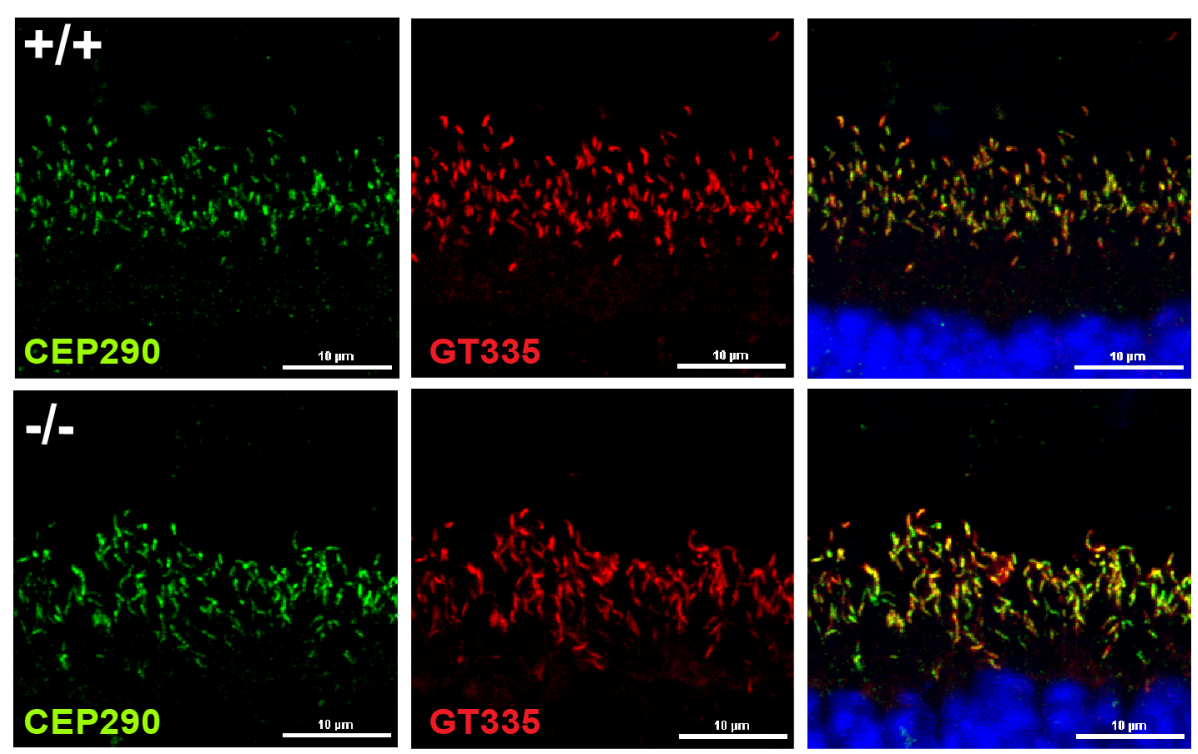

Supplemental Figure 9. CEP290 and RPGRIP localization unaffected with loss of ARL2BP. (A and B) Retinal sections from wildtype (+/+) and knockout (-/-) littermates at P16 showing localization of (A) RPGRIP (red), and Rootletin (green), and (B) CEP290 (green), and GT335 (marker for glutamylated RPGR, red). (scale bar $=2.5 \mu \mathrm{m})$. 


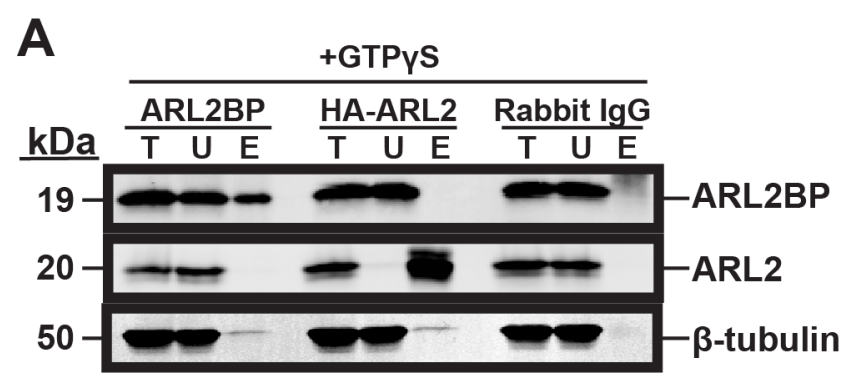

Supplemental Figure 10. Interaction between ARL2BP and ARL2 not observed in retinal extracts.

(A) Immunoprecipitation of ARL2BP or HA-tagged ARL2 using retinal lysates from transgenic mice expressing constitutively active HA-tagged ARL2 (ARL2-Q70L) at P13. Far left panel was a pull-down with ARL2BP antibody, middle panel was a pull down with HA antibody, and the Rabbit IgG pull-down was the negative control. $\mathrm{T}=$ total protein, $\mathrm{U}=$ Unbound fraction, $\mathrm{E}=$ eluate. Molecular weight in kilodaltons $(\mathrm{kDa})$ is indicated on the left. 


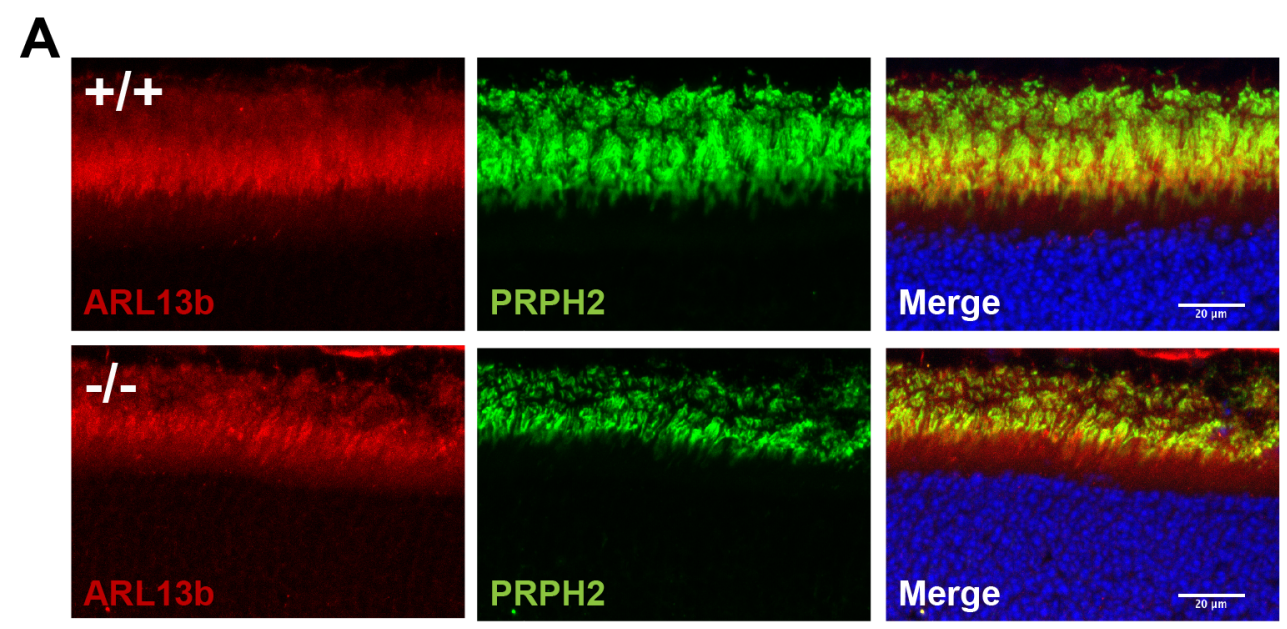

Supplemental Figure 11. ARL13b localization unaffected with loss of ARL2BP.

(A) Retinal sections from wildtype (+/+) and knockout (-/-) littermates at P16 showing localization of ARL13b (red) and PRPH2 (green). (scale bar $=20 \mu \mathrm{m})$. 
Supplemental Table 1. List of Antibodies

\begin{tabular}{|c|c|c|c|c|}
\hline Antibody & Catalog \# & Area stained & $\begin{array}{l}\text { Dilution - } \\
\text { Retina }\end{array}$ & $\begin{array}{c}\text { Dilution - } \\
\text { Immunoblot }\end{array}$ \\
\hline Rabbit anti-Pde6 $\beta$ & Thermo Fisher PA1-722 & Outer Segment & $1: 1000$ & $1: 2000$ \\
\hline $\begin{array}{l}\text { Rabbit anti- } \\
\text { PRPH2 } \\
\text { (Peripherin) }\end{array}$ & $\begin{array}{c}\text { Gift from Dr. Andrew } \\
\text { Goldberg, Oakland } \\
\text { University }\end{array}$ & Outer Segment & $1: 1000$ & $1: 2000$ \\
\hline $\begin{array}{l}\text { Mouse anti- } \\
\text { CNGA }\end{array}$ & $\begin{array}{c}\text { UC Davis/NIH } \\
\text { NeuroMab Facility }\end{array}$ & Outer Segment & $1: 500$ & $1: 1000$ \\
\hline $\begin{array}{l}\text { Mouse anti-4D2 } \\
\text { (Rhodopsin) }\end{array}$ & $\begin{array}{l}\text { Gift from Dr. Bob } \\
\text { Molday, University of } \\
\text { British Columbia }\end{array}$ & Outer Segment & $1: 500$ & $1: 2000$ \\
\hline Chicken anti-RP1 & $\begin{array}{l}\text { Gift from Dr. Eric } \\
\text { Pierce, Harvard } \\
\text { University }\end{array}$ & Axoneme & $1: 500$ & $1: 1000$ \\
\hline $\begin{array}{c}\text { Guinea pig anti- } \\
\text { MAK }\end{array}$ & Wako 012-26441 & Axoneme & $1: 500$ & $1: 1000$ \\
\hline $\begin{array}{l}\text { Mouse anti- } \\
\text { Acetylated } \\
\text { Tubulin }\end{array}$ & Sigma Aldrich T6793 & $\begin{array}{l}\text { Connecting } \\
\text { Cilium }\end{array}$ & $1: 1000$ & $1: 2000$ \\
\hline $\begin{array}{l}\text { Chicken anti- } \\
\text { Rootletin }\end{array}$ & $\begin{array}{l}\text { Gift from Dr. Tiansen } \\
\text { Li, National Eye } \\
\text { Institute }\end{array}$ & Ciliary rootlet & $1: 500$ & \\
\hline $\begin{array}{l}\text { Rabbit anti- } \\
\text { RPGR }\end{array}$ & $\begin{array}{l}\text { Gift from Dr. Hemant } \\
\text { Khanna, University of } \\
\text { Massachusetts }\end{array}$ & $\begin{array}{l}\text { Connecting } \\
\text { cilium }\end{array}$ & $1: 500$ & $1: 1000$ \\
\hline $\begin{array}{l}\text { Rabbit anti- } \\
\text { RPGRIP }\end{array}$ & $\begin{array}{l}\text { Gift from Dr. Tiansen } \\
\text { Li, National Eye } \\
\text { Institute }\end{array}$ & $\begin{array}{l}\text { Connecting } \\
\text { cilium }\end{array}$ & $1: 500$ & $1: 1000$ \\
\hline $\begin{array}{l}\text { Rabbit anti- } \\
\text { REEP6 }\end{array}$ & $\begin{array}{l}\text { Gift from Dr. Anand } \\
\text { Swaroop, National Eye } \\
\text { Institute }\end{array}$ & $\begin{array}{c}\text { Inner } \\
\text { segment/synapse }\end{array}$ & $1: 1000$ & $1: 2000$ \\
\hline $\begin{array}{l}\text { Rabbit anti- } \\
\text { Arl13b }\end{array}$ & $\begin{array}{c}\text { Protein Tech Group } \\
\text { 17711-1-AP }\end{array}$ & Primary Cilium & $1: 500$ & $1: 1000$ \\
\hline $\begin{array}{l}\text { Mouse anti- } \\
\text { Arl13b }\end{array}$ & Antibody Inc. & Primary Cilium & $1: 500$ & \\
\hline $\begin{array}{l}\text { Mouse anti- } \gamma- \\
\text { tubulin }\end{array}$ & Sigma Aldrich GTU-88 & Basal body & $1: 500$ & \\
\hline $\begin{array}{c}\text { Mouse anti- } \\
\text { polyglutamylation }\end{array}$ & Adipogen GT335 & $\begin{array}{l}\text { Connecting } \\
\text { Cilium }\end{array}$ & $1: 1000$ & $1: 2000$ \\
\hline $\begin{array}{l}\text { Rabbit anti- } \\
\text { ARL2BP }\end{array}$ & In-house & IS, BB, CC & & $1: 1000$ \\
\hline $\begin{array}{l}\text { Mouse anti- } \\
\text { ARL2BP }\end{array}$ & Santa Cruz & IS, BB, CC & $1: 250$ & \\
\hline
\end{tabular}




\begin{tabular}{|c|c|c|c|c|}
\hline Rabbit anti-ARL2 & In-house & N/A & $1: 500$ \\
\hline $\begin{array}{c}\text { Mouse anti- } \\
\text { GAPDH }\end{array}$ & Fitzgerald 10R-G109a & N/A & $1: 10,000$ \\
\hline $\begin{array}{c}\text { Mouse anti-alpha } \\
\text { tubulin }\end{array}$ & Sigma Aldrich T9026 & N/A & $1: 2000$ \\
\hline $\begin{array}{c}\text { Mouse anti-beta } \\
\text { tubulin }\end{array}$ & Sigma Aldrich T8328 & N/A & $1: 2000$ \\
\hline $\begin{array}{c}\text { Rabbit anti- } \\
\text { Syntaxin3 }\end{array}$ & Proteintech 15556 & $\begin{array}{c}\text { Inner } \\
\text { segment/synapse }\end{array}$ & $1: 1000$ & $1: 2000$ \\
\hline $\begin{array}{c}\text { Rabbit anti-opsin } \\
\text { red/green }\end{array}$ & $\begin{array}{c}\text { AB5405, EMD } \\
\text { Millipore }\end{array}$ & $\begin{array}{c}\text { Cone outer } \\
\text { segment }\end{array}$ & $1: 1000$ & $1: 2000$ \\
\hline $\begin{array}{c}\text { Rabbit anti-cone } \\
\text { arrestin }\end{array}$ & $\begin{array}{c}\text { AB15282, EMD } \\
\text { Millipore }\end{array}$ & $\begin{array}{c}\text { Cone outer } \\
\text { segment }\end{array}$ & $1: 1000$ & $1: 2000$ \\
\hline $\begin{array}{c}\text { Biotinylated } \\
\text { Peanut Agglutinin } \\
\text { (PNA) }\end{array}$ & $\begin{array}{c}\text { Vector Laboratories } \\
\text { Cone sheath }\end{array}$ & $1: 2000$ & \\
\hline $\begin{array}{c}\text { 4',6-diamindino- } \\
\text { 2-phenylindole } \\
\text { (DAPI) }\end{array}$ & Invitrogen & Nuclei & $1: 2000$ & \\
\hline $\begin{array}{c}\text { Propidium Iodide } \\
\text { RL-1072 }\end{array}$ & Vector Laboratories & Nuclei & $1: 2000$ & \\
\hline
\end{tabular}


Supplemental Table 2. List of Primers Used

\begin{tabular}{|c|c|c|}
\hline Use & Direction & Sequence (5'-3') \\
\hline $\begin{array}{l}\text { sgRNA oligonucleotide } \\
\text { template }\end{array}$ & $\mathrm{F}$ & CACCGAAATTCTGCATCAGAGGCGG \\
\hline $\begin{array}{l}\text { sgRNA oligonucleotide } \\
\text { template }\end{array}$ & $\mathrm{R}$ & AАACCCGCCTCTGATGCAGAATTTC \\
\hline sgRNA with T7 promotor: & $\mathrm{F}$ & $\begin{array}{l}\text { TTAATACGACTCACTATAGGGAAATTCT } \\
\text { GCATCAGAGGCGG }\end{array}$ \\
\hline Sequencing & $\mathrm{F}$ & GCCACTCCTGACCAGTTACC \\
\hline Sequencing/Genotyping & $\mathrm{R}$ & GGAACTAGAGGGGCACAA \\
\hline Genotyping & $\mathrm{F}$ & /IRD700/TTGGACCGGCACCTAATC \\
\hline
\end{tabular}




\title{
Chapter 3
}

Mutations in ARL2BP cause syndromic infertility in humans and mice.

\author{
Abigail R. Moye, ${ }^{1,2, *}$, Nicola Bedoni ${ }^{4, *}$,Jessica Cunningham ${ }^{3}$, Eric Tucker $^{3}$, Peter \\ Mathers ${ }^{1,2,3}$, Virginie Peter ${ }^{4}$, Liliana P. Paris ${ }^{5}$, Luísa Coutinho-Santos ${ }^{5}$, Peter Camacho ${ }^{5}$, \\ Madeleine G. Purcell ${ }^{6}$, Abbie C. Winkelmann ${ }^{6}$, James A. Foster ${ }^{6}$, Carlo Rivolta ${ }^{4,7, *}$, and \\ Visvanathan Ramamurthy ${ }^{1,2,3, *}$ \\ ${ }^{1}$ Departments of Ophthalmology, ${ }^{2}$ Department of Biochemistry, and ${ }^{3}$ Rockefeller Neurosciences \\ Institute, West Virginia University, Morgantown, WA 26506, USA. ${ }^{4}$ Department of \\ Computational Biology, University of Lausanne, Lausanne, Switzerland. ${ }^{5}$ Instituto de \\ Oftalmologia Dr Gama Pinto, Lisbon, Portugal. ${ }^{6}$ Biology Department, Randolph-Macon College, \\ Ashland, VA 23005. ${ }^{7}$ Department of Genetics and Genome Biology, University of Leicester, \\ Leicester, United Kingdom.
}

\section{Corresponding Authors:}

Visvanathan Ramamurthy, Departments of Ophthalmology and Biochemistry; Eye Institute, One Medical Center Drive, West Virginia University, Morgantown, WV 26506-9193, USA. Phone: 304-598-6925; Email: ramamurthyv@,wvumedicine.org

Carlo Rivolta, Department of Computational Biology, University of Lausanne, Rue du Bugnon 27, 1011 Lausanne, Switzerland. Phone: +4121692 5451; Email: carlo.rivolta@unil.ch

\footnotetext{
*Equal contribution
} 


\begin{abstract}
Cilia are tiny evolutionarily conserved hair-like structures with a wide spectrum of key biological roles. Ciliary and axonemal dysfunction have been linked to the growing class of genetic disorders collectively known as ciliopathies. Many strides have been made towards deciphering the causes behind this group of diseases, which have in turn increased the knowledge base on cilia and cilia dysfunctions. One recently identified cilia-associated gene is the ADP-Ribosylation Factor Like 2 Binding Partner (ARL2BP). In this study, we have identified multiple ciliopathy-associated phenotypes associated with patient mutations in ARL2BP and in a mouse knockout model. Our research demonstrates that spermiogenesis is impaired, resulting in abnormally shaped heads, shortened sperm tails, mis-assembled sperm tail structures, and loss of axonemal doublet microtubule structure. Enlarged ventricles of the brain and situs inversus were also observed in the mouse knockout model for ARL2BP. Further findings using ARL2BP knockout mouse embryonic fibroblasts (MEFs) reveal that there is delayed depolymerization of primary cilia in the absence of ARL2BP. Our results suggest that ARL2BP is required for the structural maintenance of cilia in multiple tissues and is essential for sperm flagellum assembly. Furthermore, in association with our previous studies, we establish ARL2BP as a ciliopathy-associated gene.
\end{abstract}




\section{Introduction}

Cilia are short, protruding organelles often referred to as "signaling hubs". These microtubule-based structures are involved in diverse functional roles, reflected by the group of genetic disorders referred to as "ciliopathies", which are caused by defects in cilia structure or function (Hildebrandt et al., 2011). Ciliopathy patients display a spectrum of phenotypes, which may vary based on the gene, and moreover, on the exact genetic mutation. Cilia contain a highly organized structure, consisting of a 9+0 (immotile) or 9+2 (motile) microtubule arrangement, starting with triplet tubules at the base (basal body and transition zone), doublet tubules throughout the axoneme, and singlets at the tip (Gibbons, 1961). This core structure is retained by cilia throughout the body, yet the cilium in each tissue is modified to impart unique functionality, which contributes to the broad range of ciliopathy phenotypes. For instance, the photoreceptor cilium is attached to the continually renewing membranous stacked discs where phototransduction occurs, allowing us to perceive light (photons) (Young, 1967). Meanwhile, the cilia present in the embryonic node during gastrulation can bend and sense fluid-flow, the event responsible for the left/right-patterning of the visceral organs (Levin et al., 1995). Additionally, the sperm tail flagellum is the longest in the body $(\sim 100 \mu \mathrm{m}$ in mice) and possesses additional accessory structures not present in other cilia (illustrated in Supplemental Figure 1). The last stage of spermatogenesis, spermiogenesis, is characterized by sperm tail formation, beginning with axoneme growth and assisted by a transient microtubular-based structure, the manchette (Oakberg, 1956).

Ciliary defects have been associated with Retinitis Pigmentosa (RP), a progressive blinding disease leading to loss of retinal photoreceptor cells. Interestingly, various RP-related ciliopathies also exhibit male infertility, however they are rarely studied in unison when 
elucidating the mechanistic basis of the disease. A recent study associated nonsense mutations in the polyglutamylase gene TTLL5 with cone-rod dystrophy and male infertility (Bedoni N., 2016). In support of these findings, Ttll5 knockout mice, in addition to retinal degeneration, show flagella that are detached from the sperm head, disrupted axoneme patterns with loss of tubulin doublets, and a severe loss of motility in sperm cells (Lee et al., 2013; Sun et al., 2016). Similarly, defects in the Intraflagellar Transport Protein 27 (IFT27) are linked with RPassociated Bardet-Biedl syndrome and cause sperm malformations leading to infertility in mice (Aldahmesh et al., 2014; Zhang et al., 2017).

One protein previously linked to RP, with or without situs inversus, is ADP-Ribosylation Factor Like-Protein 2 Binding Partner (ARL2BP) (Davidson et al., 2013b; Audo et al., 2016; Fiorentino et al., 2018). In agreement with these cilia-related phenotypes, ARL2BP localizes to the connecting cilia of photoreceptor cells (Davidson et al., 2013a). Interestingly, our previous findings shows that loss of ARL2BP results in abnormal doublet microtubule structure of the axoneme and shortened cilia in photoreceptor cells (Moye et al., 2018).

In this study, we report the identification of two homozygous mutations in the gene $A R L 2 B P$ in three Portuguese patients from two consanguineous families displaying RP and male infertility. The murine knockout models for the same gene showed similar phenotypes, including retinal degeneration, immotile sperm cells and impaired spermatogenesis, as well as situs inversus and increased brain ventricular volume. Our data highlight a novel ciliopathic entity linking two structurally similar, yet functionally different, ciliary organelles - the photoreceptor and the spermatozoon, associating vision and the reproductive system. 


\section{Results}

\section{Clinical evaluation}

The index subject P1 (ID: LL1) was initially evaluated at age 40 and diagnosed with retinitis pigmentosa. Born from a consanguineous union, and originally from Portugal, he was first diagnosed with myopia at age 8 and developed night blindness and photopsia by the age of 26 (Figure 1). Over the following 10 years, he developed progressive loss of vision and bilateral constriction of the peripheral visual fields. The patient noticed a more pronounced reduction in visual acuity at age 37 , associated with development of onset photophobia (both eyes), and later, metamorphopsia in the right eye.

At age 42 , his best corrected visual acuity (BCVA) was 0.2 in the right eye (OD) and 0.25 in the left eye (OS). Slit-lamp examination revealed moderate opacities of the crystalline lenses and generalized chorioretinal atrophy, including punched-out lesions, affecting the periphery and the posterior pole, upon dilated fundus examination (Figure 1A). Scarce pigment deposits were visible in the peripheral retina as well as bilateral optic disc pallor and attenuated retinal vessels (characteristic triad of retinitis pigmentosa). Fluorescence angiography showed generalized window defects and atrophy of the photoreceptor/pigment epithelium complex.

Fundus autofluorescence showed spotty areas of hypo-autofluorescence outside of the vascular arcades and a central ring of hyper-autofluorescence in the posterior pole (Figure 1).

On the last examination, at age 43, marked progression of peripheral visual field constriction was noted in both eyes, which was more severe in the OD (limited to the central 1015 degrees). Spectral domain optical coherence tomography (SD-OCT) revealed pronounced atrophy of the retinal layers, with consequent enhanced visualization of the choroidal vessels 
(Figure 1). Electroretinograms (ERG) showed no response to light stimuli, either from cone or from rod photoreceptor cells (data not shown).

The patient's past medical history was remarkable for cardiac arrhythmia under bisoprolol treatment, as well as infertility. Due to unsuccessful conception, a full spermiogram analysis was performed, the diagnosis resulting in a oligo-teratozoospermia (reduced sperm concentration and abnormal head shape) and severe asthenozoospermia (complete absence of motility). Table 1 summarizes this analysis, as compared to the reference normal values described by the World Health Organization (Cooper et al., 2010). The causes of infertility were investigated by exploring possible occurrence of deletions in the $\mathrm{Y}$ chromosome, as described for the Sertoli cell-only syndrome (OMIM: 305700). The presence of 21 genetic markers (STS) on chromosome Yq and two markers on Yp (Supplemental Table 1) was verified, therefore excluding this possibility. A fresh sperm sample, originally collected for immunofluorescence analysis for the present study, showed that most sperm heads were without flagellum, and approximately $10 \%$ of cells had a shorter flagellum (Figure $4 \mathrm{~A}$ )

Patient P2 (ID: LL89), a woman, was diagnosed with a classical form of Retinitis Pigmentosa at the age of 36 years. The patient was born from a consanguineous union between first degree cousins, of Portuguese origins. The patient's initial visual complaints started at the age 11 years, with photophobia, and progressed to night blindness and photopsia by the age of 25 , as well as constriction of the peripheral visual field by the age of 28 . Disease progression led to a sharp reduction in visual acuity at age 38 years and loss of color vision by the age of 40 . She underwent cataract surgery at age 40 and 48 . At 50 she developed metamorphopsia in the OS. Her paternal aunt P3 and her uncle P4 were also diagnosed with RP, showing similar progression and manifestations. There is no clinical history of other ciliopathic disorders in this family, with 
the exception of $\mathrm{P} 4$, whose past medical history was positive for chronic bronchitis, infertility and multiple myeloma (which ultimately led to the patient's death prior to this study, preventing further phenotypic analysis). At the last ophthalmologic examination, patient P2 showed BCVA of 0.3 in the OD, 0.16 in the OS, and severe constriction of the peripheral visual field, with bilateral tunnel-like vision, restricted to the 5 degrees central. Dilated fundus examination revealed attenuated retinal vessels, pale optic discs (Figure 1B) and a marked generalized chorioretinal atrophy in the posterior pole and in the periphery, with areas of complete atrophy in the inferior peripheral region of the right eye (RE). Mild to moderate mottled pigment deposition in the retinal periphery, along with some irregular white patches in the posterior pole near the superior vessels was also observed. SD-OCT revealed pronounced atrophy of the outer retinal layers and the presence of a foveal cyst. Fundus autofluorescence was notable for generalized hypo-autofluorescence with a wide hyper-autofluorescent ring in the posterior pole (Figure 1).

None of the patients in this study had self-reported respiratory conditions or subjective complaints (except for P4), metabolic disturbances, situs inversus or skeletal abnormalities.

\section{Identification of pathogenic variants in the $A R L 2 B P$ gene}

The DNA of P1 was initially screened for mutations in the TTLL5 gene, due to its association to a similar phenotype, but the results were negative. Following whole-exome sequencing (WES), the data were analyzed using an internal in-silico pipeline assessing variant

frequency in the general population, quality, and predicted impact at the protein level. This filtering resulted in 7 homozygous changes, of which 6 residing in autozygous regions (Figure 2). Among the 6 variants, a single-nucleotide substitution disrupting the canonical consensus donor splice site downstream of exon 3 in ARL2BP (NM_012106.3:c.207+1G>A) was identified. 
P2 was studied according to the same methodology, and 20 of the 23 rare homozygous variants resulting from our pipeline were located in autozygous regions. These included a 4-bp deletion in the coding sequence of $A R L 2 B P$ exon 1, resulting in a frameshift and in the creation of an early stop codon at the beginning of exon 2 (c.33_36delGTCT:p.Phe13ProfsTer15) (data not shown).

\section{Patient P1 aberrant splicing}

RT-PCR analysis was performed on cDNA derived from P1 sperm RNA, in comparison to RNA from a healthy control donor. Gel electrophoresis revealed the absence of a band corresponding to the expected PCR product in P1, and the appearance of a fragment of inferior molecular weight. Sanger sequencing of this latter fragment showed that in P1's sperm $A R L 2 B P$ transcripts were abnormally spliced, and that c. $207+1 \mathrm{G}>\mathrm{A}$ caused the skipping of exon 3 , the fusion of exon 2 with exon 4 , and the shift of the canonical reading frame, leading in turn to a premature termination

\section{ARL2BP localization in human sperm}

To determine the role for ARL2BP in spermiogenesis, the final stages of spermatogenesis, we investigated the localization of ARL2BP within the sperm cell. In the human control sample, ARL2BP localized at the base of the flagellum, as well as equatorial zone of the head (Figure 4A). In P1's sample, we observed most of the sperm heads separated from their tails, though some intact sperms had a shortened tail. In the majority of cells from patient P1, staining for ARL2BP was non-specific with faint background (Figure 4A). In about $2-5 \%$ of the cells from P1, staining was similar to that of the control sample, 
possibly reflecting the residual expression of ARL2BP in this patient (data not shown).

In agreement with findings from human samples, ARL2BP was found in the head-tail connecting apparatus, and the principal piece in murine sperm (Figure 4B). In contrast, no signal was observed in KO murine sperm, demonstrating the specificity of the antibody used (Figure 4B).

\section{Immotile sperm with stubby tails and a decreased sperm cell count in P1 and in $A r l 2 b p$ KO mice.}

Concurrent with the identification of the male patient, we discovered that male Arl $2 b p$ KO mice were infertile, as they were not yielding any litters during the generation of the murine Arl2bp KO model to study blindness (Moye et al., 2018). Therefore, we examined sperm motility with live imaging and found that the KO sperm were immotile, in agreement with the human patient phenotypic description (Supplemental Video 1 and 2). Of note, testis size and weight were comparable between WT and KO (Figure 5A and B). Morphological analysis of the testes by $\mathrm{H} \&$ E staining displayed normal spermatogenesis in $\mathrm{KO}$ animals. However, sperm released into the lumen appeared impaired, with a smaller lumen area, an absence of sperm tails, and an increase in residual bodies (RB) (Figure 5C). Inspection of the murine KO sperm revealed a drastically decreased sperm cell count (Figure 5D), and additional light microscopy images of cauda epididymis sperm revealed that Arl2bp KO sperm had gross morphological defects, including numerous detached heads and tails, kinked tails at the connecting piece, stubby tails, abnormal heads, and cytoplasmic bulges attached to the tails (Figure 5E). These results are consistent with the oligo-teratozoospermia reported in the male patient and the morphology of the sperm as detected by light microscopy (Figure 4A). 


\section{Failure to complete spermiogenesis in the absence of ARL2BP.}

The severity of azoospermia in $\mathrm{P} 1$ and $\mathrm{KO}$ mice spurred the investigation into the morphology and formation of the sperm tail and accessory structures using established markers. We first examined the sperm tail core (axoneme) using microtubule-associated markers. Despite severe loss in protein levels in Arl2bp KO animals (Figure 6A), Acetylated Tubulin (AcTu) and Glutamylated Tubulin (GluTu, GT335) localized to the sperm tail in WT and $\mathrm{KO}$ animals (Figure 4B and 6D). Moreover, testes cross-sections from $\mathrm{KO}$ mice revealed a further irregularity in tail shapes (spiraled) with GluTu staining (Figure 6C). In addition, Retinitis Pigmentosa GTPase Regulator (RPGR) and Sperm Flagellar Protein 2 (SPEF2), both axoneme associated proteins, were barely present in $\mathrm{KO}$ murine sperm, with diminished and spotty staining (Figure 6E). These findings show that loss of ARL2BP does not interrupt the initiation of microtubular axoneme growth. However, shortened axonemes and a decrease in axoneme-associated protein localization to the sperm tail indicates that axoneme formation is impaired.

To determine if the assembly of the accessory structures is normal in the absence of ARL2BP, we assessed sperm tails using markers such as A-Kinase Anchoring Protein 4 (AKAP4, fibrous sheath, FS) and Outer Dense Fiber Protein 1 (ODF1, outer dense fiber). Both markers were absent in murine $\mathrm{KO}$ sperm, though present in murine $\mathrm{KO}$ testes lysates (Figure 6A and E). Additionally, both forms of AKAP4 were at the expected distribution in WT animals (Johnson et al., 1997), with most of the soluble, non-assembled precursor to AKAP4 (pro-AKAP4, 82kDa) in the testes samples, while the majority present in the sperm lysates was the phosphorylated form of AKAP4 assembled into the FS (109kDa) (Figure 6A). In contrast, both forms of the protein were present in the $\mathrm{KO}$ testes, with neither present in the sperm 
lysates (Figure 6A). Supporting this, staining in KO testes sections revealed retention of AKAP4 in the residual bodies shed during spermiogenesis, and an absence of AKAP4 from sperm tails. In comparison, WT displayed AKAP4 in both residual bodies and luminal sperm tails (Figure 6B). Conversely, we observed staining for Heat-Shock Protein 60kDa (HSP60, mitochondrial sheath) in murine KO sperm (though reduced) (Figure 6E). This finding was independently confirmed by the presence of pyruvate dehydrogenase protein (Pyr Deh., mitochondrial sheath) in sperm lysates (Figure 6A). Furthermore, staining of WT and KO testes cross-sections using Mitotracker showed that the development of mitochondria in elongating spermatids was present (Figure 6C), indicating the formation of a mitochondrial sheath. All together, these results demonstrate a failure to complete spermiogenesis by the inability to form the outer dense fiber layer or assemble the fibrous sheath in $A r l 2 b p \mathrm{KO}$ animals.

\section{Inconsistent acrosome structure with lack of ARL2BP.}

ARL2BP staining in the sperm head and the presence of abnormally shaped sperm heads in the male patient and $\mathrm{KO}$ mice prompted our investigation of acrosome development throughout spermatogenesis. The acrosome starts to spread like a cap around the nucleus in stages VI-VII (cap phase), yet the KO displayed acrosomal irregularities exemplified by clusters of acrosomal granules instead of the cap structure (Figure 8A and B). Furthermore, at later stages in spermatogenesis, there were instances of irregularly shaped acrosomes surrounding regularly shaped nuclei. Figure $8 \mathrm{C}$ shows acrosome caps in $\mathrm{KO}$ testes at stages VIII-XI that are more oval instead of round, with a flimsier appearance. While at stage XII, there were a few misshapen acrosomes displaying a "molar tooth" shape (Figure 8D). Of note, most acrosomes on elongating spermatids at steps 14-16 (stage II-VIII) in KO testes appear 
normal, suggesting that ARL2BP is not crucial for acrosome formation.

\section{ARL2BP is essential for flagellar microtubule structure.}

Extensive analysis of ultrastructural images from WT and $\mathrm{KO}$ murine testes and epididymis tissue corroborated the decreased sperm count and lack of sperm tail formation in the KO. Spermatogenesis appeared to be relatively normal through early tail formation in spermiogenesis, including formation of the manchette with centrally located centrioles; however, significant abnormalities in head, neck, and tail ultrastructure were noted in later stages of spermiogenesis after step 10 (Figure 7). In sperm from testis sections, the basal plate and capitulum were present in the neck, but segmented columns were either absent or severely disrupted (Figure $7 \mathrm{~F}_{2}$ ). The mitochondrial sheath contained centrally located mitochondria, but they were not properly organized (Figure 7F). The outer dense fibers and fibrous sheath were in disarray, though present, which is consistent with the protein levels observed for ODF1 and AKAP4 (Figure 7E). This contrasts with the WT sperm tails, which demonstrated the organized structure illustrated in Supplemental Figure 1 (Figure 7A and B). Furthermore, longitudinal and cross-sections of Arl2bp KO sperm tails revealed that microtubules were present in parallel arrays in the proximal tail region, but they did not form the canonical $9+2$ axoneme arrangement (Figure 7C). Microtubules were not paired at all (singlets) and some seemed to be incomplete tubules. There was also evidence of uneven thickness in the tail staining with tubulin (GT335 and AcTu, Figure 4B and 6D), which coincides with the uneven microtubules presented in the ultrastructural images. Nevertheless, most tubules were associated with electron-dense material that appears to be outer dense fibers and/or fibrous sheath. These accessory structures were not properly organized and were scattered in various portions of the tail (Figure 7E and F). 
Additionally, Arl2bp KO sperm at stage 9 displayed manchette (Figure 7D). Sperm at later stages in development (once manchette is disassembled) were not detected.

\section{ARL2BP is necessary for left-right patterning in mice.}

Human patients, as well as $\mathrm{KO}$ animals, were further examined for additional phenotypes typically observed in ciliopathies. The human patients in this study did not report any symptoms associated with impaired cilia in the trachea or kidney. This finding is corroborated by our observations of normal tracheal cilia beat frequency and morphologically normal kidneys in Arl2bp KO mice (data not shown). However, we observed a high number of KO mice displaying situs inversus or heterotaxy, an asymmetric L/R positioning of the internal organs caused by defects in the nodal cilia of developing embryos (Figure 9A). The association of ARL2BP mutations in humans with situs inversus is variable, and the patients reported in this study did not possess situs inversus. However, a previous study identified one patient with a mutation in ARL2BP with situs inversus (Davidson et al., 2013a). In mice, this phenotype is much less variable, as most $A r l 2 b p \mathrm{KO}$ animals had situs inversus (55\%), with $28 \%$ possessing heterotaxy of either the heart or stomach (Figure 9B). Furthermore, tracking and statistical analysis revealed that the number of KO's produced from Heterozygous $\mathrm{x}$ Knockout or from Heterozygous x Heterozygous parents did not follow Mendelian ratios when examined from full-term litters or from embryonic day 13.5 (e13.5) (chi square value of 39.19, $\mathrm{p}>0.001$, and chi square value of $7,0.01>\mathrm{p}>0.005$, respectively). However, litters examined from e7.5 did follow Mendelian ratios (chi square value of 0.96) suggesting that partial embryonic lethality is mediated by the absence of ARL2BP at or near the time of gastrulation (after day e7.5) (Figure 8C). These results establish ARL2BP's essential role in nodedetermined laterality during development. 


\section{Cerebral ventricular volume affected with loss of ARL2BP.}

An additional ciliated tissue investigated in the Arl2bp KO mice was the cerebral ventricles. These are fluid-filled cavities in the brain that contain ciliated tufts on the apical surface of ependymal cells. There are four interconnected cavities, including the two lateral ventricles and third ventricle in the forebrain which are connected to the fourth ventricle in the brain stem by the cerebral aqueduct present in the midbrain. The motile cilia in these cavities are responsible for the circulation of cerebrospinal fluid (CSF) from the lateral ventricles, where it is produced, to the surrounding space of the brain and spinal cord. Disruption of the CSF flow results in enlarged ventricles and hydrocephaly (build-up of CSF in the ventricles). The gross morphology of the rest of the brain appears similar to WT in size and shape. White matter axon tracts and gray matter volume appeared consistent between genotypes, indicating that deletion of $A r l 2 b p$ does not result in gross structural brain malformations. Remarkably, a 45 fold increase in lateral ventricular volume was observed in the brains of $\mathrm{KO}$ mice compared to WT littermates using micro-CT scans (Figure 9). Of note, the third ventricle also appeared enlarged in Arl2bp KO mice, however we were not able to accurately quantify this due to problems with segmentation from the surrounding space. Additionally, there was no noticeable difference in the cerebral aqueduct or fourth ventricle between WT and Arl2bp KO mice, nor was there any sign of obstruction in the cerebral aqueduct or fourth ventricle, which is commonly linked to hydrocephaly. Interestingly, the majority of KO mice did not appear hydrocephalic, a phenotype associated with many ciliopathies, with no obvious behavioral or motor deficits associated with this increase in ventricular volume. Though the larger ventricular volume points to a role for ARL2BP in CSF flow regulation, a detailed behavioral analysis 
would need to be completed to confirm the observation that these structural changes do not cause any behavioral deficits.

\section{MEFs display shortened primary cilia in the absence of ARL2BP.}

To further investigate the role for ARL2BP, mouse embryonic fibroblasts (MEFs) were generated from Arl2bp KO mice and WT littermates. Primary cilia were induced in MEFs after removal of serum from the growth media. After 48 hours of serum starvation, the percentage of ciliated cells was comparable between WT and KO (Figure 10C and D). MEFs lacking ARL2BP possessed significantly shorter cilia (average of 2! m) than WT MEFs (average of 2.7! m) (Figure 10A and B). To determine if the cilia present in Arl2bp KO MEFs had difficulty in primary cilia depolymerization or re-entry into the cell cycle, cilia resorption was assessed throughout 24 hours of serum addition on cilia-induced cells. Interestingly, after 2 hours of serum addition, a significantly higher percentage of KO MEFs retained their cilia than what was observed in WT (Figure 10C and D). Further observation of KO and WT MEFs revealed that cilia resorption was comparable from 6 to 24 hours (Figure 10D). These results indicate that loss of ARL2BP affects the initial depolymerization of primary cilia. 


\section{Discussion}

The splice variant in our first index patient, as well as a different nucleotide substitution in the same position, were recently published as causative for RP only (and situs inversus in one patient) (Davidson et al., 2013a; Fiorentino et al., 2018). Consistent with these findings, our previous murine studies show that loss of ARL2BP results in abnormal doublet microtubule structure of the axoneme and shortened cilia in photoreceptor cells, causing blindness (Moye et al., 2018). Our current study sought to understand the mechanism behind ARL2BP dysfunction by examining both patients and mice. With our findings, we validate the past association with the blinding disease RP, and bring the first evidence of ARL2BP involvement in spermatogenesis. We thereby prove that additional cilia-related phenotypes might originate from the loss of full- length ARL2BP, which point to the need for ARL2BP in cilia and flagella structure and maintenance.

Since its discovery, the function of ARL2BP has remained largely elusive. Initial characterization was established by its interaction with ARL2, a member of the ARF-like (ADP ribosylation factors) family which are a group of small GTP-binding proteins belonging to the RAS superfamily $(27,28)$. Past publications have established that dysfunctional ARL2BP impacts the photosensory cilium in humans and mice (Davidson et al., 2013b; Moye et al., 2018). Experiments in human retinal pigment epithelial cells showed that ARL2BP knockdown with small interfering RNA caused reduced cilia length (Davidson et al., 2013b), and similarly, germline deletion of ARL2BP in mice showed shortened photoreceptor cilia with impairment of ciliary doublet microtubule structure (open inner junction, IJ) (Moye et al., 2018). A recent report using constitutively active ARL2 (Q70L mutation) in retina demonstrates a defect in outer segment morphology with rapid loss of 
photoreceptor function (Wright, et. al., 2018; in press). However, Arl2bp KO does not phenocopy ARL2 and in addition our attempts to show this interaction in sperm or retina have been unfruitful (data not shown). It is possible that ARL2BP plays a role independent of ARL2.

\section{ARL2BP is essential for sperm flagellar midpiece formation in humans and mice.}

Spermatogenesis begins in the basal compartment at the outer edge of the seminiferous tubules and continues inward until fully formed spermatids are released into the lumen and on to the epididymal tissues. The last stage, spermiogenesis, is characterized by sperm tail formation, beginning with axoneme growth and assisted by a transient microtubular structure, the manchette (Oakberg, 1956).

Without ARL2BP, sperm tails fail to elongate. Morphological analysis with Transmission Electron Microscopy (TEM) revealed that the 9+2 axoneme structure was disorganized, with no detectable doublet microtubules (DMT). The outer dense fibers and fibrous sheath structures still accumulate around the tail during spermatogenesis but fail to assemble appropriately. This coincides with the loss of ODF1 (ODF) and AKAP4 (FS) staining, and the increase of residual bodies present in testes of $\mathrm{KO}$ animals, an indicator of failed spermiogenesis. Furthermore, the increased levels of phosphorylated AKAP4 in the testes instead of the sperm of KO mice points to the inability to properly assemble the FS present throughout the principal piece. The presence of p-AKAP4 in the testes lysates is likely related to the fibrous sheath fragments seen in the cytoplasmic bulges present in electron micrograph images of the testes, as well as the AKAP4containing residual bodies. The misassembled FS is also linked to the irregular axoneme structure, as the ODFs attach directly to their corresponding DMTs in the principal piece 
(Lindemann and Gibbons, 1975; Olson and Linck, 1977). FS assembly occurs in a distal to proximal direction, following formation of the axoneme. Without the stable interaction and growth of the DMTs and ODFs there is not creation of a true "distal end". Likely, the smaller, disorganized clumps that show FS periodicity and rib-like appearance in the ultrastructural images is due to the inability of the FS anlagen (precursor) to assemble correctly along the distal axoneme. Furthermore, AKAP4 processing and FS assembly is dependent on the formation and proper localization of the annulus. This is a septin ring-structure formed during spermiogenesis that travels to its position between the mid- and principal pieces after midpiece formation is complete (46). If the midpiece forms improperly, the annulus cannot localize appropriately, resulting in abnormal processing of AKAP4 and a failure to complete spermiogenesis.

These defects in FS, and thereby principal piece formation, are accompanied by issues in midpiece formation. In the midpiece, the ODF does not directly bind to the microtubule doublets, but it is held in place by, and in early spermatogenesis attached to, the surrounding mitochondrial sheath (MS) (Lesich et al., 2014; Lindemann, 2016). The lack of a properly assembled MS could be attributed to the poorly assembled ODFs. Therefore, it is possible that without ARL2BP, the malformation in the microtubule structure causes impaired assembly of all periaxonemal structures and a failure to complete spermiogenesis. This is evident by the lack of principal pieces (which assembles last) in sperm tails from the KO model and in the majority of sperms from the human sample.

\section{Putative role for ARL2BP in intra-manchette transport (IMT)}

The manchette is a transient microtubule- and F-actin-based structure formed during spermiogenesis. The formation and assembly of the MS, ODF, FS, and acrosome is mediated by 
intra manchette transport (IMT), a process resembling intraflagellar transport (IFT) (Zhang et al., 2016; Lehti and Sironen, 2017; Liu et al., 2017). Given that the MS, ODF, FS and acrosome assembly depends on ARL2BP, and that the manchette forms in Arl2bp KO sperm, we hypothesize that IMT is impaired or that there is a delay in the manchette disassembly. This hypothesis is supported by animal models that lack SPEF2, a protein required for the axonemal central pair in sperm flagellum, and MEIG1, a protein required for spermiogenesis. Loss of either of these proteins results in abnormal head and tail formation, which in both cases are linked to an elongated or delayed manchette (Zhang et al., 2009; Salzberg et al., 2010; Sironen et al., 2010; Sironen et al., 2011; Li et al., 2015a; Lehti et al., 2017). Though it is unlikely ARL2BP directly binds with SPEF2 or MEIG1, the abnormally shaped heads and impaired assembly of accessory structures with loss of these proteins is similar, indicating the ARL2BP may be involved in IMT or manchette disassembly. Furthermore, we showed ARL2BP localizing in the equatorial zone of the acrosome, as well as abnormal acrosome formation in the Arl2bp KO sperm. In mammals, proteins localizing at the equatorial segment of the acrosome are involved in the initiation of sperm-oocyte fusion (Yanagimachi, 1994; Wolkowicz et al., 2003). However, it remains unclear if ARL2BP has any direct role in the egg fertilization process. Taken together with the data observed from the WT and Arl2bp KO MEFs, the delay in cilia depolymerization closely relates to the hypothesis that ARL2BP is involved in manchette disassembly. Therefore, we hypothesize that ARL2BP is essential for depolymerization of microtubules in the manchette and MEF primary cilium. 


\section{ARL2BP is involved in organ laterality.}

To date, only one patient with defective ARL2BP was reported to have situs inversus (Davidson et al., 2013b). This laterality defect was also observed in KO mice, but not in patients from the two families of this study, who have normal organ position (situs solitus). L/Rasymmetry is determined by the mono-ciliated cells in the embryonic node during gastrulation. There is a leftward-fluid flow generated in this region by motile monocilia, which bend the immotile cilium of the crown cells and signals for asymmetrical protein expression related to eventual organ placement (McGrath et al., 2003; Babu and Roy, 2013). Therefore, if this fluid flow is disrupted, the laterality of the organs will be affected. It was shown in an elegant study by Nonaka, et. al. 2002 that complete reversal of the flow (rightward) resulted in complete situs inversus (Nonaka et al., 2002). However, what determines situs inversus vs other forms of laterality placements are not known and is thought to be stochastic. Since the axonemes in photoreceptors and sperm tails are shorter with loss of ARL2BP, it is likely that nodal cilia are also shorter in the KO animal. Interestingly, there was an abnormally high incidence (55\%) of situs inversus, compared with normal laterality or heterotaxia in the KO mice. Throughout the breeding process however, we noticed a significant decrease in the expected number of KO animals after laterality had been determined (e7.5). Therefore, we hypothesize that some KO embryos possessing heterotaxia died embryonically. Since different forms of heterotaxia are related to embryonic lethality (heart malformations, congenital heart disease), this could be possible (Li et al., 2015b; Narasimhan and Roy, 2015). This could also explain why only one patient identified with $A R L 2 B P$ mutations possesses situs inversus, while the incidence in the mouse model is much higher. 


\section{ARL2BP is not essential for the function of multi-ciliated cells.}

Multi-ciliated cells are present in the ventricles of the brain, throughout the trachea, and in the fallopian tubes. Patients with mutations in $A R L 2 B P$ were not reported to have any symptoms related to defects in these cilia (sinusitis, otitis media, hydrocephaly, or female infertility). Among patients of this study, only P4 was reported with chronic bronchitis. The late age of onset of this respiratory issue and the fact that the patient is presently deceased, makes it difficult to discern possible association of this to mutated ARL2BP-driven ciliary defects. To note, the mouse model also did not display any related symptoms. Interestingly however, the trachea of KO mice possessed tufts of normal cilia, immotile cilia, and cilia with uncoordinated beating (data not shown). Likewise, the ventricular volumes in $\mathrm{KO}$ mouse brains were significantly increased but neither of these defects were enough to cause a discernible phenotype. Therefore, we consider that ARL2BP is not required for the function of multi-ciliated cells, though it does appear to be necessary for robust performance of these cilia.

Notably, it is plausible that many adult ciliopathy patients with no reported hydrocephaly could possess asymptomatic hydrocephalus (symptoms are chronic headache, visual problems, clumsiness, and fogginess), which would go unnoticed as a disease symptom unless proper questions were asked and imaging was performed (Cowan et al., 2005). Furthermore, hydrocephalus is a rarely reported phenotype of ciliopathies, unlike the higher incidence of hydrocephalus reported in murine models for ciliopathies (Ferkol and Leigh, 2012; Finn et al., 2014). Interestingly, however, when trying to sonographically diagnose Primary Cilia Dyskinesia (PCD) patients, slightly enlarged ventricles were observed in an embryo with situs inversus (Wessels et al., 2003). These observations point to two concerns in comparing mouse models of ciliopathies with human patients. One, it appears that mice ventricular ependymal cilia are more 
susceptible to disturbances, and therefore hydrocephaly. Two, human patients not presenting with overt childhood hydrocephalus might be overlooked in diagnosing ciliopathies.

\section{Conclusions}

With our findings, we validate the past association of $A R L 2 B P$ mutations with the blinding disease RP, and bring the first evidences of ARL2BP involvement in spermatogenesis. We thereby prove that additional cilia-related phenotypes might originate from ARL2BP deficiency, with manifestations that are similar in human and mouse. Furthermore, we provide a first insight into the disease mechanisms associated with $A R L 2 B P$ mutations in relationship to defective ciliogenesis, pointing to an essential role for this protein in the maintenance of normal structure and homeostasis of cilia and flagella. 


\section{Materials and Methods}

\section{Patients and controls}

Patients were recruited from the Instituto de Oftalmologia Dr. Gama Pinto in Lisbon, Portugal. DNA was extracted from peripheral blood leukocytes (subjects P1 and P2), and from saliva (subject P3), after obtaining written informed consent. RNA was extracted from a sperm sample from patient P1. A control sperm and DNA sample were provided by a healthy donor.

\section{Clinical evaluation}

Ophthalmologic examination included assessment of Best Corrected Visual Acuity (BCVA), slit-lamp examination, dilated fundus examination, fundus photography, visual fields, and optical coherence tomography (OCT). Full-field ERGs were also recorded, following the International Society for Clinical Electrophysiology of Vision (ISCEV) protocol. Semen analysis (subject P1) was carried out by standard procedures by an andrology laboratory, and according to WHO guidelines (Cooper et al., 2010). Additional clinical features were assessed based on patients' clinical history.

\section{Whole-exome sequencing (WES)}

WES was performed using $2 \mu \mathrm{g}$ DNA derived from peripheral blood mononuclear cells. Protein-coding regions were captured using the HiSeq Rapid PE Cluster Kit v2, and an Illumina HiSeq 2500 instrument was used for paired-end sequencing. Single nucleotide variants and small insertions and deletions were detected using the Genome Analysis Tool Kit (GATK v4.0) software package, using the Best Practice Guidelines identified by the developers (DePristo et 
al., 2011). The pathogenicity of the detected genetic variants was assessed after functional annotation through ANNOVAR (Wang et al., 2010). Genomic regions with high homozygosity were determined using proprietary software.

\section{Sanger sequencing validation, PCR, and RT-PCR}

Primer pairs for $A R L 2 B P$ exons and flanking intron boundaries were designed using the CLCbio Genomics Workbench (Qiagen). PCR amplification was performed in a $10 \mu \mathrm{l}$ total volume containing 2 ng genomic DNA or cDNA, 1x GoTaq buffer, $0.1 \mathrm{mM}$ dNTPs, $10 \mu \mathrm{M}$ of each primer, and $5 \mathrm{U} / \mu 1$ of GoTaq polymerase (Promega). PCR products were purified using ExoSAP-IT, USB, or extracted from agarose gel using Nucleospin Gel and PCR Clean-up (Macherey-Nagel). Sanger sequencing was performed by a service provider (Fasteris, http://www.fasteris.com/). Reverse transcription was performed on $1 \mu \mathrm{g}$ RNA using a mix of random primers and the GoScript Reverse Transcription Protocol (Promega).

\section{Immunoblotting}

Mice were euthanized by $\mathrm{CO}_{2}$ inhalation and testes were dissected and separated from epididymis and fat. Testis samples were weighed and immediately frozen in liquid nitrogen. Prior to analyses, the samples were homogenized in phosphate buffered saline (PBS, with protease inhibitor cocktail (Thermo Fisher \#A32955)) and a NanoDrop spectrophotometer was used to measure protein concentrations. Samples were analyzed by SDS-PAGE gel, followed by transfer onto polyvinylidene difluoride membranes. After blocking the membranes with western blot blocking buffer (LiCor 004864 Classic) for $30 \mathrm{~min}$ at room temperature, they were incubated with the primary antibodies overnight at $4^{\circ} \mathrm{C}$. The membranes were then washed in 
PBST (PBS with $0.1 \%$ Tween-20) 3 times for 5 minutes ( 3 X $5 \mathrm{~min}$ ) at room temperature before incubation in secondary antibody (goat anti-rabbit Alexa 680, goat anti-rat Alexa 680, or goat anti-mouse Alexa 800) for $30 \mathrm{~min}$ at room temperature. After 3 x 5 minutes of washes with PBST, membranes were scanned using Odyssey Infrared Imaging System.

\section{Immunofluorescence}

For testis, the anesthetized animal was perfusion fixed with $4 \%$ PFA and then the testis was dissected out. This was followed by incubation in $30 \%$ sucrose/PBS overnight at $4{ }^{\circ} \mathrm{C}$. Afterward, testes were incubated in a 1:1 mixture of 30\% sucrose in PBS and OCT (Cryo Optimal Cutting Temperature Compound, Sakura) for $1 \mathrm{hr}$, and flash frozen in OCT. Staining was performed following the same protocol as detailed below. For sperm, the animal was euthanized by $\mathrm{CO}_{2}$ inhalation, and the epididymis was collected. The tissue was then minced, and sperm were allowed to swim out for 5-10 minutes (in the case of knockout, entire volume of liquid was collected) and collected into an Eppendorf tube. $100 \mu 1$ of sperm suspension was added to a Superfrost Plus slide and allowed to completely dry by placing the slides on a hot plate (setting 3, Corning Hot Plate). Cells were fixed in 4\% PFA for 15 minutes followed by three 5 minute washes in PBS. Ice-cold methanol was then added for 2 minutes, followed by three 5 minute washes in PBS. The cells were then blocked overnight at $4{ }^{\circ} \mathrm{C}$ (PBS with 5\% goat sera, $0.5 \%$ TritonX-100, $0.05 \%$ sodium azide). The next day, the cells were incubated with primary antibody for 2 hours at RT, followed by three 5 minute washes in PBS. The cells were then incubated with secondary antibody for 1 hour, followed by three 5 minute washes in PBS. They were then mounted with ProLong Gold anti-fade reagent and viewed on a Nikon confocal microscope. 
Immunofluorescence for human P1 and control sperm samples was performed as described in previously-published protocols (Nishimura et al., 2011; Bedoni N., 2016). The same antiARL2BP primary antibody described above was used in the human sperm staining experiments, in addition to a secondary goat anti-rabbit antibody conjugated with Alexa Fluor 488 (Invitrogen). Pictures were taken on a Zeiss LSM 780 confocal microscope.

\section{Cell counting}

Mice were euthanized by $\mathrm{CO}_{2}$ inhalation and epididymal sperm were dissected out and placed in $1 \mathrm{ml}$ PBS. Sperm were collected, spun at 1500 RPM for 3 minutes and re-suspended in $50 \mu 1$ of PBS (knockout) or 4\% paraformaldehyde (wild type; and further diluted 1:10 in PBS after 5 min). Sperm cells were counted using a hemocytometer.

\section{MEF Cells}

Embryos were harvested at E13.5 and separated from each other and the placenta in PBS. The tissues were minced with sterile razor blades. The tissues were then trypsinized with $6 \mathrm{ml}$ total of 0.25\% Trypsin/EDTA. $7 \mathrm{ml}$ of MEF media (catalog \# 10-013-CV from Corning) DMEM containing glucose, pyruvate, and L-glut $+15 \% \mathrm{FBS}, 1 \%$ Pen/Strep) was then added to each tube and spun down at 1200 RPM for 8 minutes. Cells were then added to a $100 \mathrm{~mm}$ dish containing $25 \mathrm{ml}$ of MEF media and allowed to grow overnight at $37^{\circ} \mathrm{C}$ with $5 \% \mathrm{CO}_{2}$. Cells were maintained

for up to 4 passages. To induce cilia formation, cells were grown to $90 \%$ confluency and serum starved for 48 hours. Depolymerization of cilia was done by the addition of sera (after 48 hours of starvation), and cells were collected for staining after 2 hours, 6 hours, 12 hours, or 24 hours of serum addition. 
For immunocytochemistry, cells were fixed with $4 \%$ PFA for 15 min (or $-20^{\circ} \mathrm{C}$ Methanol for 2 minutes for centrosomal staining, acetylated tubulin and pericentrin), washed with PBS 3 x 5 minutes, and blocked for 30-60 minutes (PBS with 5\% goat sera, 0.5\% TritonX-100, 0.05\% sodium azide). The remaining steps were performed in the same way as outlined in the immunofluorescence section.

\section{Transmission Electron Microscopy}

Mice were euthanized by $\mathrm{CO}_{2}$ asphyxiation and testes were dissected and separated from epididymis and fat. Testes were decapsulated and 1-3mm pieces were placed into $4 \%$ paraformaldehyde and $2 \%$ glutaraldehyde in $0.2 \mathrm{M}$ cacodylate buffer overnight at $4^{\circ} \mathrm{C}$. Sperm were also collected from epididymal tissues and placed in the same fixative in the same conditions. Testis and sperm samples were then post-fixed in $1 \%$ osmium tetroxide, dehydrated in a series of increasing ethanol concentration, and embedded in Embed812 resin (Electron Microscopy Sciences, Hatfield, PA). Thin sections were cut and stained with UranyLess (EMS, Hatfield, PA) and photographed on a Hitachi HT7700 TEM (NSF grant \#1229184).

\section{Micro-CT Scans}

Transcardial perfusions were performed on anesthetized 60 day old mice with $4 \%$ paraformaldehyde. The brain was carefully dissected out of the skull and fixed in $4 \%$ paraformaldehyde for 2 days at $4^{\circ} \mathrm{C}$. The brains were then transferred to stability buffer $(4 \% \mathrm{w} / \mathrm{v}$ paraformaldehyde ( $\mathrm{pH} 7.2), 4 \% \mathrm{w} / \mathrm{v}$ acrylamide, $0.05 \% \mathrm{w} / \mathrm{v}$ bis-acrylamide, $0.25 \% \mathrm{w} / \mathrm{v} \mathrm{VA} 044$ initiator, $0.05 \% \mathrm{w} / \mathrm{v}$ Saponin, in $1 \mathrm{xPBS}$ ) for 3 days at $4^{\circ} \mathrm{C}$, and then underwent nitrogen desiccation, followed by a 3 hour incubation at $37^{\circ} \mathrm{C}$. After a two-day staining in $0.1 \mathrm{~N}$ iodine, the 
brains were embedded into $3 \%$ agarose for imaging. The brains were imaged on a Bruker SkyScan 1272 MicroCT scanner (Cu $0.11 \mu \mathrm{m}$ filter, $1500 \mathrm{~ms}$ exposure, and $8 \mu \mathrm{m}$ resolution), and 3D reconstruction of ventricular volume was performed with Seg3D software.

\section{Statistics}

All data are presented as mean \pm standard error margin. Immunoblots and ciliated cell counts were analyzed by unpaired, two-tailed $t$ test $(n=3)$. For cilia measurements, staining was performed in triplicate with 100 cilia measured for each and data were visualized with the ggplot2 package in $\mathrm{R}$ version 3.3.2. Image and densitometry analysis were performed using ImageJ 1.50i. For ciliated cell counting, staining was performed in triplicate with 100 cells (and their cilia) counted for each. Mendelian ratios were analyzed using a chi square test with 1 degree of freedom. Chi square values of 3.84 or higher are statistically significant $(\mathrm{p}>0.05)$.

\section{Antibodies}

Information relative to antibody origin and working dilutions are presented below.

\begin{tabular}{|c|c|c|c|c|}
\hline Antibody & Catalog \# & Area stained & \begin{tabular}{|c|} 
Dilution \\
IF
\end{tabular} & $\begin{array}{c}\text { Dilution } \\
\text { Immunobl } \\
\text { ot }\end{array}$ \\
\hline $\begin{array}{l}\text { Mouse anti-Acetylated } \\
\text { Tubulin }\end{array}$ & Sigma Aldrich T6793 & $\begin{array}{l}\text { Connecting } \\
\text { Cilium }\end{array}$ & $1: 1000$ & $1: 2000$ \\
\hline Rabbit anti- RPGR & $\begin{array}{c}\text { Gift from Dr. } \\
\text { Hemant Khanna, University } \\
\text { of } \\
\text { Massachusetts }\end{array}$ & $\begin{array}{l}\text { Connecting } \\
\text { cilium }\end{array}$ & $1: 500$ & $1: 1000$ \\
\hline Rabbit anti- Arl13b & $\begin{array}{c}\text { Protein Tech Group } \\
17711-1-\text { AP }\end{array}$ & Primary Cilium & $1: 1000$ & $1: 1000$ \\
\hline Mouse anti- Arl13b & Antibody Inc. & Primary Cilium & $1: 500$ & N/A \\
\hline Mouse anti- $\gamma$ tubulin & $\begin{array}{c}\text { Sigma Aldrich } \\
\text { GTU-88 }\end{array}$ & Basal body & $1: 200$ & N/A \\
\hline Rabbit anti-Pericentrin & Abcam 4448 & Basal body & $1: 200$ & N/A \\
\hline
\end{tabular}




\begin{tabular}{|c|c|c|c|c|}
\hline $\begin{array}{c}\text { Mouse anti- } \\
\text { glutamylation }\end{array}$ & Adipogen GT335 & $\begin{array}{l}\text { Connecting } \\
\text { Cilium }\end{array}$ & $1: 1000$ & $1: 2000$ \\
\hline Rabbit anti-ARL2BP & In-house, (Moye et al., 2018) & IS, BB, CC & $1: 500$ & $1: 1000$ \\
\hline Mouse anti-GAPDH & Fitzgerald 10R-G109a & N/A & N/A & $1: 10,000$ \\
\hline $\begin{array}{l}\text { Mouse anti-alpha } \\
\text { tubulin }\end{array}$ & Sigma Aldrich T9026 & N/A & N/A & $1: 2000$ \\
\hline Mouse anti-beta tubulin & Sigma Aldrich T8328 & N/A & N/A & $1: 2000$ \\
\hline Mouse anti-AKAP4 & sc-135827 & Fibrous Sheath & $1: 250$ & $1: 500$ \\
\hline Mouse anti-ODF1 & sc-390152 & ODF & $1: 250$ & $1: 500$ \\
\hline Rabbit anti-SPEF2 & HPA040343 & $\mathrm{CP}$ & $1: 250$ & N/A \\
\hline Mouse anti-HSP60 & $\begin{array}{c}\text { Enzo Life Sciences } \\
\text { SPA-806 }\end{array}$ & MS & $1: 250$ & N/A \\
\hline $\begin{array}{c}\text { Rabbit anti- } \\
\text { Pyruvate } \\
\text { Dehydrogenase }\end{array}$ & $\begin{array}{l}\text { Thermo Fisher } \\
\text { PA5-21536 }\end{array}$ & Mitochondria & $\mathrm{N} / \mathrm{A}$ & $1: 1000$ \\
\hline Mitotracker & Life Technologies M7510 & Mitochondria & $\begin{array}{c}100 \mathrm{nM} \\
\text { in media }\end{array}$ & N/A \\
\hline $\begin{array}{c}\text { Biotinylated } \\
\text { Peanut Agglutinin } \\
\text { (PNA) }\end{array}$ & Vector Laboratories & Acrosome & $1: 2000$ & N/A \\
\hline $\begin{array}{l}\text { 4',6-diamindino- 2- } \\
\text { phenylindole } \\
\text { (DAPI) }\end{array}$ & Invitrogen & Nuclei & $1: 2000$ & N/A \\
\hline
\end{tabular}

\section{Study approval}

Our research has been conducted in accordance with the tenets of the Declaration of Helsinki and was approved by the Institutional Review Boards of our respective Organizations. Informed consent was obtained from all patients prior to the sample collection. Institutional Animal Care and Use Committee of the West Virginia University approved all experimental procedures involving animals conducted in this study. 


\section{Supplemental Data}

Supplemental Data include two videos. 


\section{Authors contributions}

ARM and NB designed, conducted, acquired, and analyzed the experiments related to the mouse and human samples, respectively. LCS, LPP, and PC are the medical scientists who visited the patients. ARM, NB, CR, VR, LCS, LPP, and VP wrote the manuscript. ARM and PM performed live imaging of sperm. ARM and JC performed the CT-scans on the mice, JC and ET analyzed the data. MGP, ACW, and JAF performed the ultrastructural imaging and analysis. VR and CR supervised the research. 


\section{Acknowledgements}

The authors thank Dr. Peter Stoilov, Fatimah Matalkah, and Dr. Elena Pugacheva (West Virginia University) for help with data collection and analysis, the Imaging Core for use of video microscopes and Micro CT-scanner (West Virginia University), the Pathology Core for help with the preparation/processing of tissues (West Virginia University), and Cecelia Lo for insightful discussions (University of Pittsburgh). This work was supported by USA National Institutes of Health Grants R01 EY028035 (to VR), R01 EY025536 (to VR and Peter Stoilov), R21 EY027707 (to VR), and West Virginia Lions and Lions Club International Foundation, as well as grants from the Swiss National Science Foundation to CR [grant number:176097], NSF MRI \#1229184 to JAF. 


\section{References}

Aldahmesh, M.A., Li, Y., Alhashem, A., Anazi, S., Alkuraya, H., Hashem, M., Awaji, A.A., Sogaty, S., Alkharashi, A., Alzahrani, S., Al Hazzaa, S.A., Xiong, Y., Kong, S., Sun, Z., and Alkuraya, F.S. (2014). IFT27, encoding a small GTPase component of IFT particles, is mutated in a consanguineous family with Bardet-Biedl syndrome. Hum Mol Genet 23, 3307-3315.

Audo, I., El Shamieh, S., Méjécase, C., Michiels, C., Demontant, V., Antonio, A., Condroyer, C., Boyard, F., Letexier, M., Saraiva, J.P., Blanchard, S., Mohand-Saïd, S., Sahel, J.A., and Zeitz, C. (2016). ARL2BP mutations account for $0.1 \%$ of autosomal recessive rod-cone dystrophies with the report of a novel splice variant. Clinical Genetics 92, 109-111.

Babu, D., and Roy, S. (2013). Left-right asymmetry: cilia stir up new surprises in the node. Open Biol 3, 130052.

Bedoni N., H.-W.L., Vaclavik V., Tran V.H., Farinelli P., Balzano S., Royer-Bertrand B., ElAsrag M.E., Bonny O., Ikonomidis C., Litzistorf Y., Nikopoulos K., Yioti G.G., Stefaniotou M.I., McKibbin M., Booth A.P., Ellingford J.M., Black G.C., Toomes C., Inglehearn C.F., Hoyng C.B., Bax N., Klaver C.C., Thiadens A.A., Murisier F., Schorderet D.F., Ali M., Cremers F.P., Andréasson S., Munier F.L., Rivolta C. (2016). Mutations in the polyglutamylase gene TTLL5, expressed in photoreceptor cells and spermatozoa, are associated with cone-rod degeneration and reduced male fertility. Hum Mol Genet 25, 4546-4555.

Cooper, T.G., Noonan, E., von Eckardstein, S., Auger, J., Baker, H.W.G., Behre, H.M., Haugen, T.B., Kruger, T., Wang, C., Mbizvo, M.T., and Vogelsong, K.M. (2010). World Health Organization reference values for human semen characteristics* + . Human Reproduction Update $16,231-245$.

Cowan, J.A., McGirt, M.J., Woodworth, G., Rigamonti, D., and Williams, M.A. (2005). The syndrome of hydrocephalus in young and middle-aged adults (SHYMA). Neurological Research $27,540-547$.

Davidson, A.E., Schwarz, N., Zelinger, L., Stern-Schneider, G., Shoemark, A., Spitzbarth, B., Gross, M., Laxer, U., Sosna, J., Sergouniotis, P.I., Waseem, N.H., Wilson, R., Kahn, R.A., Plagnol, V., Wolfrum, U., Banin, E., Hardcastle, A.J., Cheetham, M.E., Sharon, D., and Webster, A.R. (2013a). Mutations in ARL2BP, encoding ADP-ribosylation-factor-like 2 binding protein, cause autosomal-recessive retinitis pigmentosa. Am J Hum Genet 93, 321-329.

Davidson, A.E., Schwarz, N., Zelinger, L., Stern-Schneider, G., Shoemark, A., Spitzbarth, B., Gross, M., Laxer, U., Sosna, J., Sergouniotis, P.I., Waseem, N.H., Wilson, R., Kahn, R.a., Plagnol, V., Wolfrum, U., Banin, E., Hardcastle, A.J., Cheetham, M.E., Sharon, D., and Webster, A.R. (2013b). Mutations in ARL2BP, encoding ADP-ribosylation-factor-like 2 binding protein, cause autosomal-recessive retinitis pigmentosa. American Journal of Human Genetics 93, 321329.

DePristo, M.A., Banks, E., Poplin, R.E., Garimella, K.V., Maguire, J.R., Hartl, C., Philippakis, A.A., del Angel, G., Rivas, M.A., Hanna, M., McKenna, A., Fennell, T.J., Kernytsky, A.M., Sivachenko, A.Y., Cibulskis, K., Gabriel, S.B., Altshuler, D., and Daly, M.J. (2011). A 
framework for variation discovery and genotyping using next-generation DNA sequencing data. Nature genetics 43, 491-498.

Ferkol, T.W., and Leigh, M.W. (2012). Ciliopathies: the central role of cilia in a spectrum of pediatric disorders. The Journal of Pediatrics 160, 366-371.

Finn, R., Evans, C.C., and Lee, L. (2014). Strain-Dependent Brain Defects in Mouse Models of Primary Ciliary Dyskinesia with Mutations in Pcdp1 and Spef2. Neuroscience 277, 552-567.

Fiorentino, A., Yu, J., Arno, G., Pontikos, N., Halford, S., Broadgate, S., Michaelides, M., Carss, K.J., Raymond, F.L., Cheetham, M.E., Webster, A.R., Downes, S.M., Hardcastle, A.J., for the, N.-B.R.D.C., and the, U.K.I.R.D.C. (2018). Novel homozygous splicing mutations in ARL2BP cause autosomal recessive retinitis pigmentosa. Molecular Vision 24, 603-612.

Gibbons, I.R. (1961). The relationship between the fine structure and direction of beat in gill cilia of a lamellibranch mollusc. The Journal of Biophysical and Biochemical Cytology 11, 179205.

Hildebrandt, F., Benzing, T., and Katsanis, N. (2011). Ciliopathies. The New England journal of medicine 364, 1533-1543.

Johnson, L.R., Foster, J.A., Haig-Ladewig, L., Vanscoy, H., Rubin, C.S., Moss, S.B., and Gerton, G.L. (1997). Assembly of AKAP82, a Protein Kinase A Anchor Protein, into the Fibrous Sheath of Mouse Sperm. Developmental Biology 192, 340-350.

Lee, G.-S., He, Y., Dougherty, E.J., Jimenez-Movilla, M., Avella, M., Grullon, S., Sharlin, D.S., Guo, C., Blackford, J.A., Awasthi, S., Zhang, Z., Armstrong, S.P., London, E.C., Chen, W., Dean, J., and Simons, S.S. (2013). Disruption of Ttll5/Stamp Gene (Tubulin Tyrosine Ligaselike Protein 5/SRC-1 and TIF2-associated Modulatory Protein Gene) in Male Mice Causes Sperm Malformation and Infertility. The Journal of Biological Chemistry 288, 15167-15180.

Lehti, M.S., and Sironen, A. (2017). Formation and function of sperm tail structures in association with sperm motility defects. Biol Reprod 97, 522-536.

Lehti, M.S., Zhang, F.-P., Kotaja, N., and Sironen, A. (2017). SPEF2 functions in microtubulemediated transport in elongating spermatids to ensure proper male germ cell differentiation. Development 144, 2683-2693.

Lesich, K.A., de Pinho, T.G., Dang, L., and Lindemann, C.B. (2014). Ultrastructural evidence that motility changes caused by variations in ATP, $\mathrm{Mg} 2+$, and ADP correlate to conformational changes in reactivated bull sperm axonemes. Cytoskeleton 71, 649-661.

Levin, M., Johnson, R.L., Stern, C.D., Kuehn, M., and Tabin, C. (1995). A molecular pathway determining left-right asymmetry in chick embryogenesis. Cell 82, 803-814.

Li, W., Tang, W., Teves, M.E., Zhang, Z., Zhang, L., Li, H., Archer, K.J., Peterson, D.L., Williams, D.C., Jr., Strauss, J.F., 3rd, and Zhang, Z. (2015a). A MEIG1/PACRG complex in the 
manchette is essential for building the sperm flagella. Development (Cambridge, England) 142, 921-930.

Li, Y., Klena, N.T., Gabriel, G.C., Liu, X., Kim, A.J., Lemke, K., Chen, Y., Chatterjee, B., Devine, W., Damerla, R.R., Chang, C., Yagi, H., San Agustin, J.T., Thahir, M., Anderton, S., Lawhead, C., Vescovi, A., Pratt, H., Morgan, J., Haynes, L., Smith, C.L., Eppig, J.T., Reinholdt, L., Francis, R., Leatherbury, L., Ganapathiraju, M.K., Tobita, K., Pazour, G.J., and Lo, C.W. (2015b). Global genetic analysis in mice unveils central role for cilia in congenital heart disease. Nature 521, 520-524.

Lindemann, C.B., and Gibbons, I.R. (1975). Adenosine triphosphate-induced motility and sliding of filaments in mammalian sperm extracted with Triton X-100. J Cell Biol 65, 147-162.

Lindemann, C.B., Lesich, Kathleen A. (2016). Functional Anatomy of the Mammalian Sperm Flagellum. Cytoskeleton 73, 652-669.

Liu, H., Li, W., Zhang, Y., Zhang, Z., Shang, X., Zhang, L., Zhang, S., Li, Y., Somoza, A.V., Delpi, B., Gerton, G.L., Foster, J.A., Hess, R.A., Pazour, G.J., and Zhang, Z. (2017). IFT25, an intraflagellar transporter protein dispensable for ciliogenesis in somatic cells, is essential for sperm flagella formation. Biol Reprod 96, 993-1006.

McGrath, J., Somlo, S., Makova, S., Tian, X., and Brueckner, M. (2003). Two populations of node monocilia initiate left-right asymmetry in the mouse. Cell 114, 61-73.

Moye, A.R., Singh, R., Kimler, V.A., Dilan, T.L., Munezero, D., Saravanan, T., Goldberg, A.F.X., and Ramamurthy, V. (2018). ARL2BP, a protein linked to retinitis pigmentosa, is needed for normal photoreceptor cilia doublets and outer segment structure. Mol Biol Cell 29, 15901598.

Narasimhan, V., and Roy, S. (2015). Cilia: Organelles at the Heart of Heart Disease. Curr Biol 25, R559-562.

Nishimura, H., Gupta, S., Myles, D.G., and Primakoff, P. (2011). Characterization of mouse sperm TMEM190, a small transmembrane protein with the trefoil domain: evidence for colocalization with IZUMO1 and complex formation with other sperm proteins. Reproduction 141, 437-451.

Nonaka, S., Shiratori, H., Saijoh, Y., and Hamada, H. (2002). Determination of left-right patterning of the mouse embryo by artificial nodal flow. Nature 418, 96-99.

Oakberg, E.F. (1956). A description of spermiogenesis in the mouse and its use in analysis of the cycle of the seminiferous epithelium and germ cell renewal. American Journal of Anatomy 99, 391-413.

Olson, G.E., and Linck, R.W. (1977). Observations of the structural components of flagellar axonemes and central pair microtubules from rat sperm. J Ultrastruct Res 61, 21-43. 
Salzberg, Y., Eldar, T., Karminsky, O.-D., Itach, S.B.-S., Pietrokovski, S., and Don, J. (2010). Meig1 deficiency causes a severe defect in mouse spermatogenesis. Developmental Biology 338, 158-167.

Sironen, A., Hansen, J., Thomsen, B., Andersson, M., Vilkki, J., Toppari, J., and Kotaja, N. (2010). Expression of SPEF2 during mouse spermatogenesis and identification of IFT20 as an interacting protein. Biol Reprod 82, 580-590.

Sironen, A., Kotaja, N., Mulhern, H., Wyatt, T.A., Sisson, J.H., Pavlik, J.A., Miiluniemi, M., Fleming, M.D., and Lee, L. (2011). Loss of SPEF2 Function in Mice Results in Spermatogenesis Defects and Primary Ciliary Dyskinesia. Biology of Reproduction 85, 690-701.

Sun, X., Park, J.H., Gumerson, J., Wu, Z., Swaroop, A., Qian, H., Roll-Mecak, A., and Li, T. (2016). Loss of RPGR glutamylation underlies the pathogenic mechanism of retinal dystrophy caused by TTLL5 mutations. Proc Natl Acad Sci U S A 113, E2925-2934.

Wang, K., Li, M., and Hakonarson, H. (2010). ANNOVAR: functional annotation of genetic variants from high-throughput sequencing data. Nucleic Acids Research 38, e164-e164.

Wessels, M.W., den Hollander, N.S., and Willems, P.J. (2003). Mild fetal cerebral ventriculomegaly as a prenatal sonographic marker for Kartagener syndrome. Prenatal Diagnosis 23, 239-242.

Wolkowicz, M.J., Shetty, J., Westbrook, A., Klotz, K., Jayes, F., Mandal, A., Flickinger, C.J., and Herr, J.C. (2003). Equatorial segment protein defines a discrete acrosomal subcompartment persisting throughout acrosomal biogenesis. Biol Reprod 69, 735-745.

Yanagimachi, R. (1994). Fertility of mammalian spermatozoa: its development and relativity. Zygote 2, 371-372.

Young, R.W. (1967). The renewal of photoreceptor cells outer segments The Journal of Cell Biology 33, 61-72.

Zhang, Y., Liu, H., Li, W., Zhang, Z., Shang, X., Zhang, D., Li, Y., Zhang, S., Liu, J., Hess, R.A., Pazour, G.J., and Zhang, Z. (2017). Intraflagellar transporter protein (IFT27), an IFT25 binding partner, is essential for male fertility and spermiogenesis in mice. Dev Biol 432, 125 139.

Zhang, Z., Li, W., Zhang, Y., Zhang, L., Teves, M.E., Liu, H., Strauss, J.F., Pazour, G.J., Foster, J.A., Hess, R.A., and Zhang, Z. (2016). Intraflagellar transport protein IFT20 is essential for male fertility and spermiogenesis in mice. Molecular Biology of the Cell 27, 3705-3716.

Zhang, Z., Shen, X., Gude, D.R., Wilkinson, B.M., Justice, M.J., Flickinger, C.J., Herr, J.C., Eddy, E.M., and Strauss, J.F., 3rd. (2009). MEIG1 is essential for spermiogenesis in mice. Proceedings of the National Academy of Sciences of the United States of America 106, 1705517060 . 


\section{Figure and Legends}

Patient P1
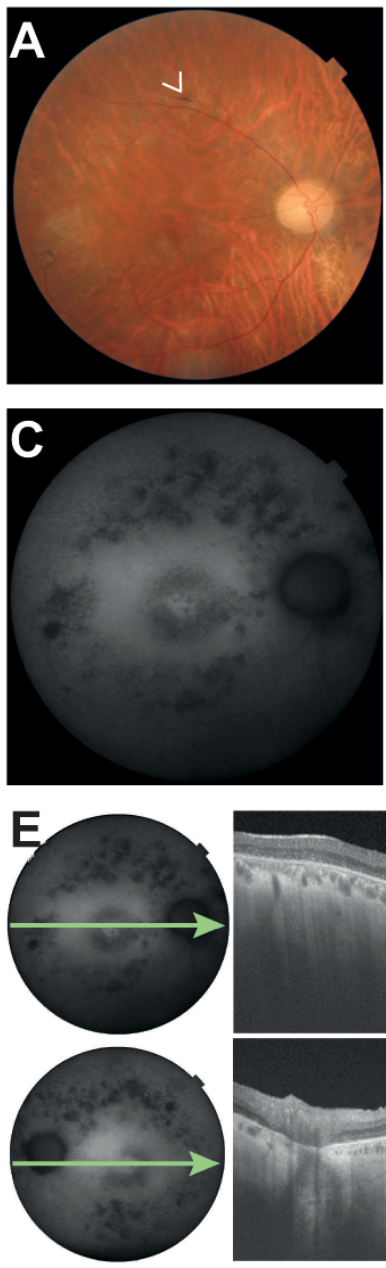
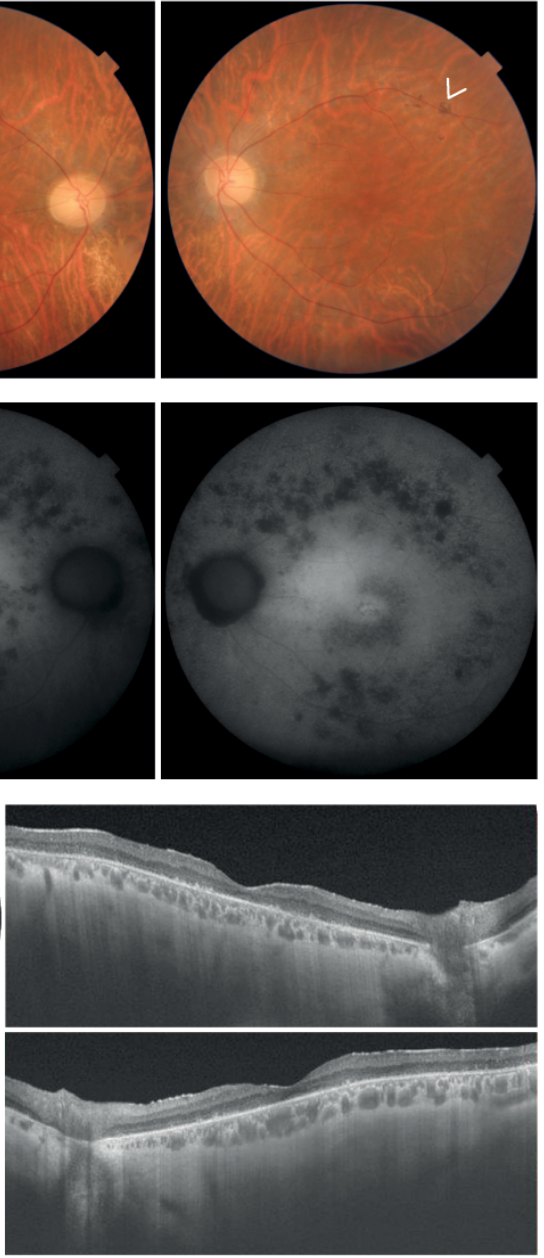

Patient P2
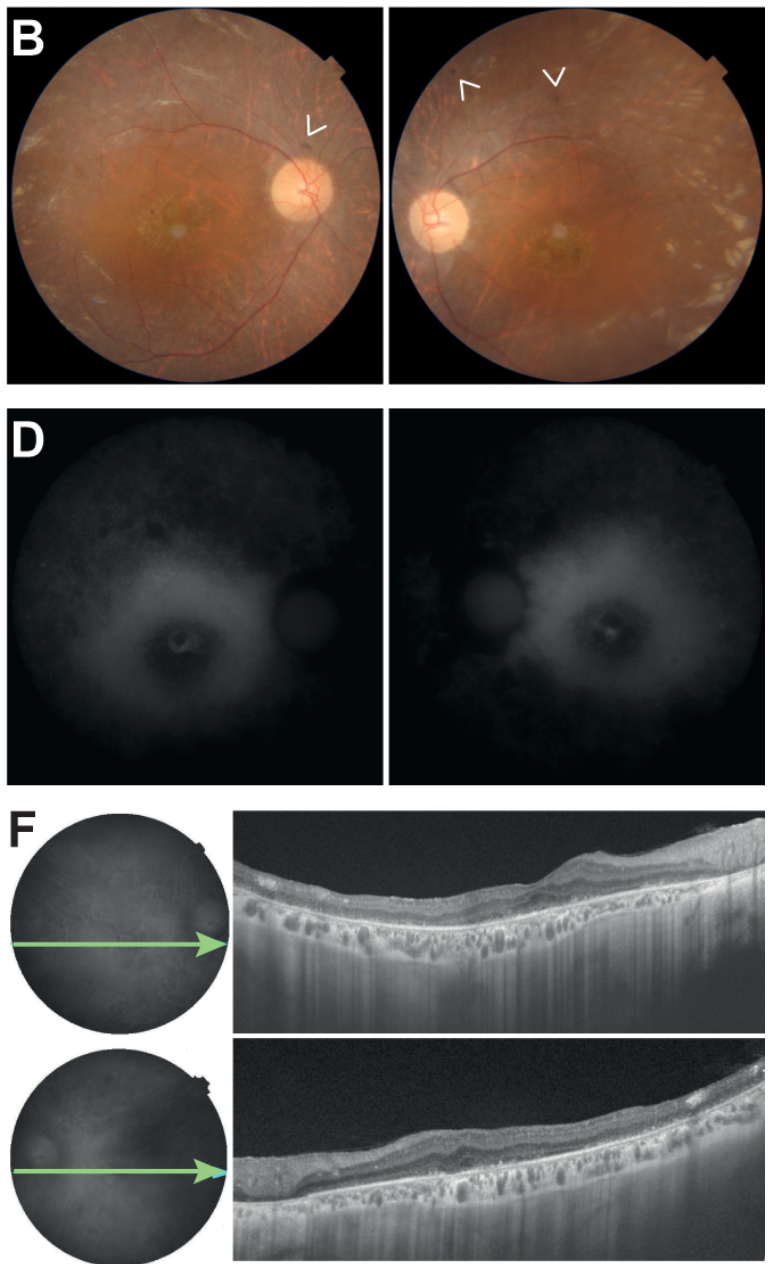

Figure 1. Findings on retinal imaging of patients $P 1$ and $P 2$.

A and B. Fundus photos of patients $\mathrm{P} 1$ and P2, respectively. P1 presents a pale optic disc, vascular thinning and retinal atrophy in the posterior pole and along the vascular arcades. Scarse pigment deposition can be found along the superior vascular arcade (as shown by the arrowheads). Similarly, P2 displays a pale optic disc, vascular thinning and marked retinal changes, with opalescent areas of the retinal tissue in the periphery and central atrophic lesion. Scarse pigment deposition can also be found adjacent to the optic nerve head and periphery (arrowheads) C and D. Fundus autofluorescence showing in P1 multiple hypoautofluorescent spots in the periphery corresponding to RPE atrophy, which in contrast is largely diffused in P2. Moreover, both patients present the typical central hyperautofluorescent ring. E and F. SD-OCT revealing in both patients diffuse retinal thinning, absent photoreceptors along with enhanced visualization of the choroidal vessels. Panels A-D: right eye followed by left eye. Panels E and F: right eye above and left eye below. 


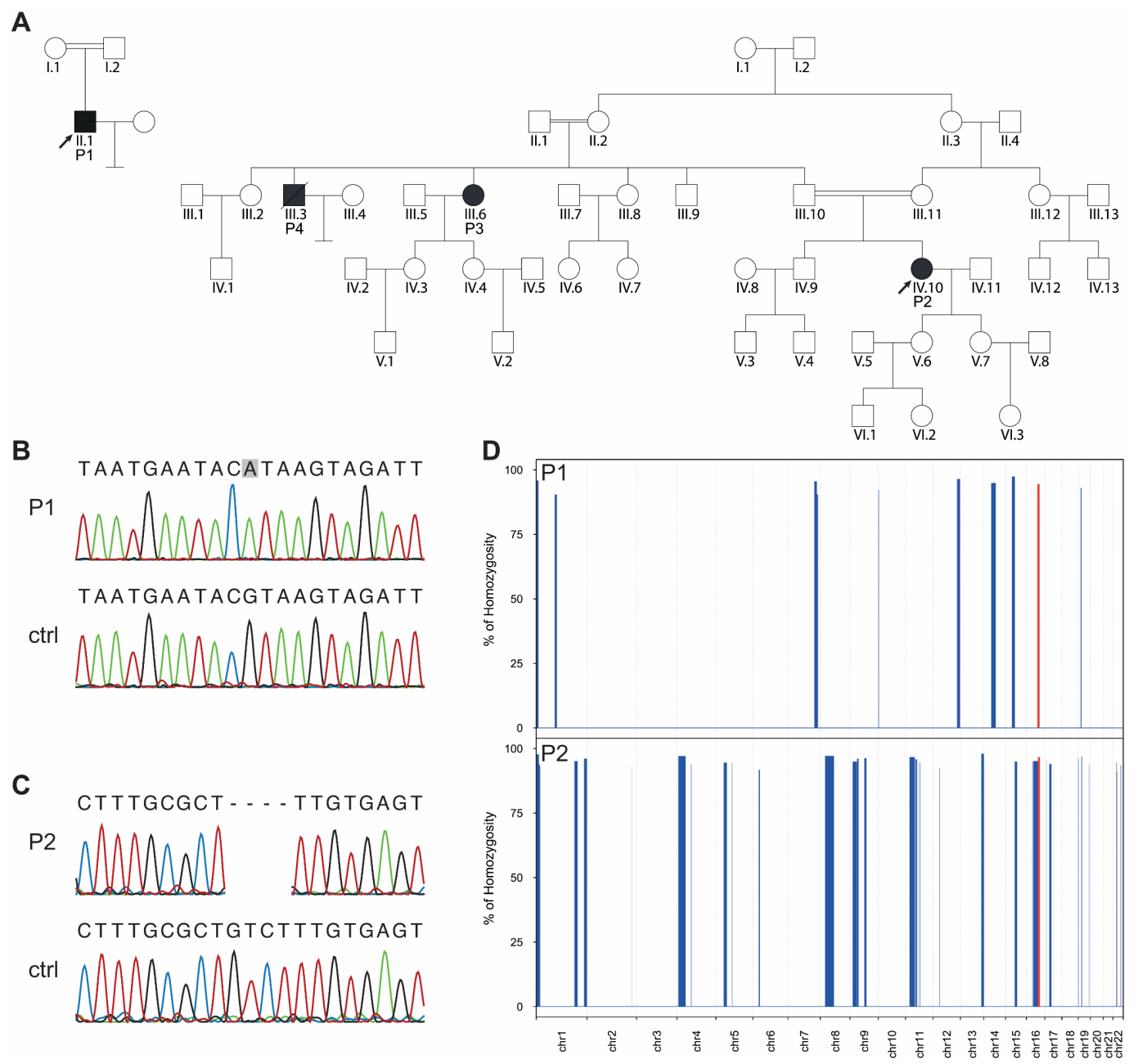

Figure 2. Pedigrees and genetic findings.

A. Pedigrees of the patients analysed in this study. DNA was available only for subjects P1, P2, and $\mathrm{P} 3$. $\mathbf{B}$ and $\mathbf{C}$. Sanger validation of the WES findings, showing the presence of a homozygous splicing mutation in patient P1 (B, NM_012106.3:c.207+1G $>$ A, leading to p.Asp35PhefsTer8), and a frameshift deletion in patients P2 and P3 (C, NM_012106.3:c.33_36delGTCT: p.Phe13ProfsTer15), alongside with relevant control sequences (ctrl). D. Exome-wide homozygosity mapping for autosomal chromosomes, using the ARMS tool (unpublished). The autozygous region containing the gene $A R L 2 B P$ is highlighted in red. 
A

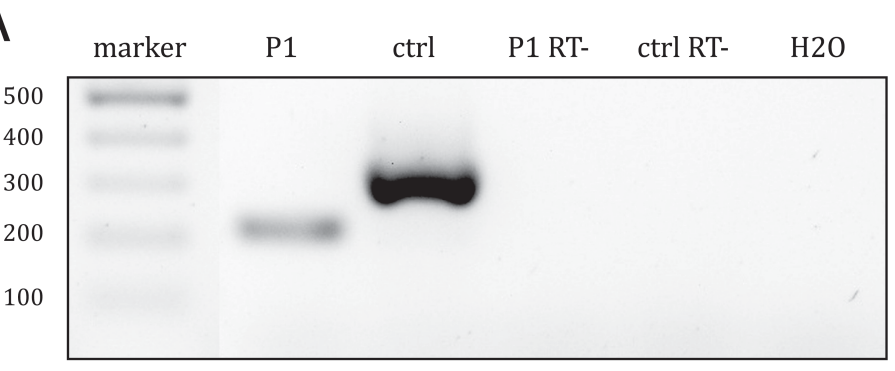

B

TTATCATGG|ATTTCTTTGG

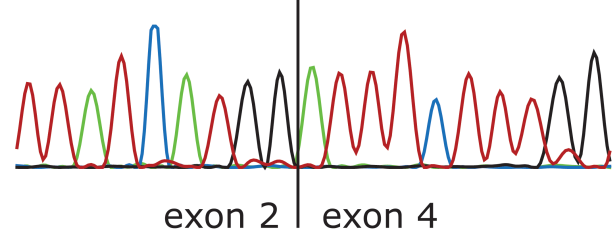

C

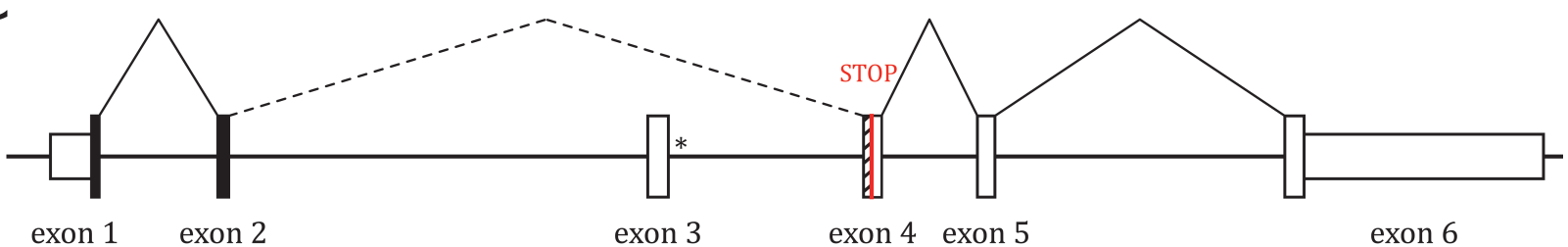

Figure 3. Effect of the splicing mutation in patient $P 1$.

A. RT-PCR on cDNA obtained from sperm-derived RNA and Sanger sequencing of the same PCR product (P1, cDNA from patient; ctrl, cDNA from a healthy control; P1 RT- and ctrl RT-, reverse transcription controls) (B). C. In-scale schematic representation of the splicing pattern resulting from the presence of the mutation. c. $207+1 \mathrm{G}>\mathrm{A}$ leads to the skipping of exon 3 and the joining of exon 2 with exon 4 , with subsequent shifting of the reading frame and creation of a premature stop codon early in exon 4 (red). The asterisk shows where the mutation is localized. The in-frame exons are black, whereas the striped portion of exon 4 is out-of-frame. 


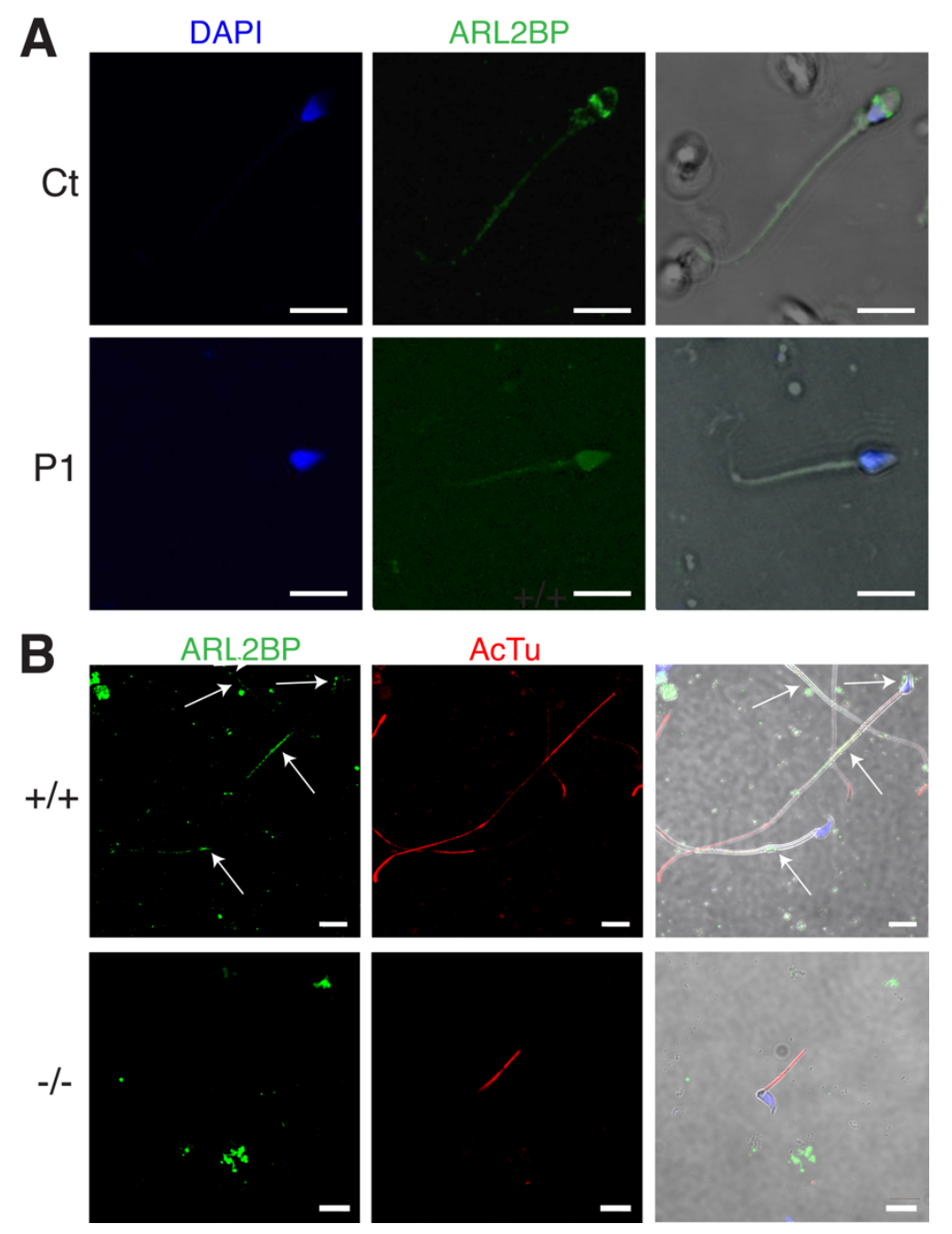

Figure 4. ARL2BP localizes to the sperm tail in humans and mice.

A. Most representative pictures: in the human control $(\mathrm{Ct})$, staining for ARL2BP is mainly at the equatorial segment of the sperm head and the principal piece of the tail, whereas in the human patient (P1) there was very diminished staining. B. WT $(+/+)$ and $\mathrm{KO}(-/-)$ murine sperm stained for ARL2BP to corroborate human sperm staining, co-stained with Acetylated Tubulin (AcTu, red). $+/+$ demonstrating localization in the sperm head, at the head-tail connecting apparatus (HTCA), the principal piece, and the annulus. Staining in the KO serves as a negative control. Scale bars $=10 \mu \mathrm{m}$. 

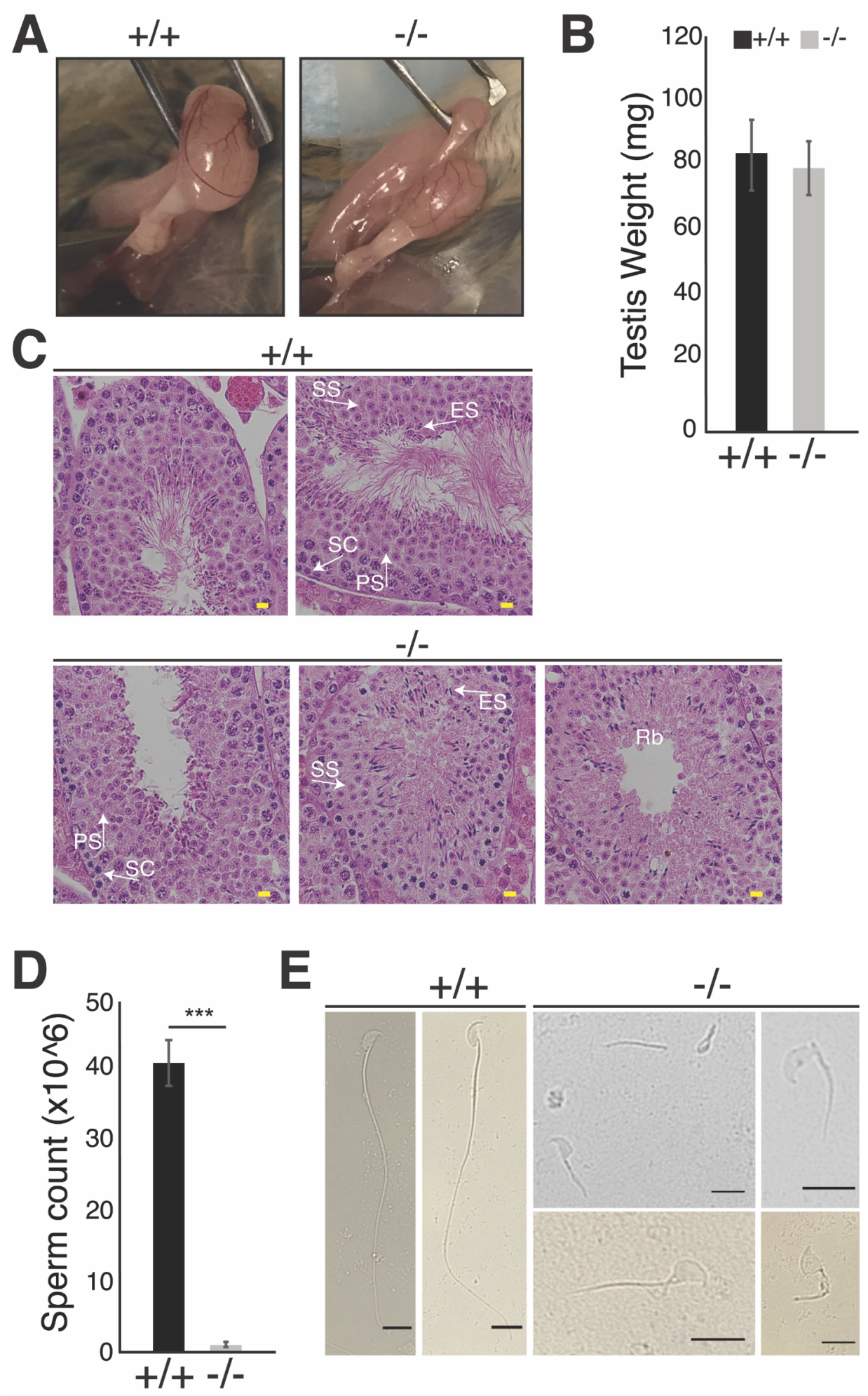

shapes, and sperm tails are shorter, detached, and retain cytoplasm. Scale bar $=10 \mu \mathrm{m}$. mice. spermatocyte, $\mathrm{SS}=$ secondary spermatocyte, mean \pm SEM.

Figure 5. Loss of

ARL2BP leads to decreased sperm count and abnormal sperm structure in

$A$ and B. Testis weight between WT $(+/+)$ and $\mathrm{KO}(-/-)$ mice are comparable, represented in $\mathbf{A}$ ) with average weights graphed in B). $n=10$. Data are represented as the mean $\pm \mathrm{SEM}$. C. H\&E sections of WT (+/+) and KO (-/-) testis, with KO testis demonstrating lack of sperm tails in the lumen and an increase in residual bodies (RB). PS=primary $\mathrm{ES}=$ elongating spermatid, $\mathrm{SC}=$ sertoli cells. Scale bar $=$ $20 \mu \mathrm{m}$. D. Graph presenting the sperm cell counts of WT $(+/+)$ and $\mathrm{KO}(-/-)$ mice. $n=3$. Data are represented as the $\mathrm{P}=0.0002$. E. Light images displaying WT sperm with normal sperm tail structure, while KO sperm show abnormal sperm head 

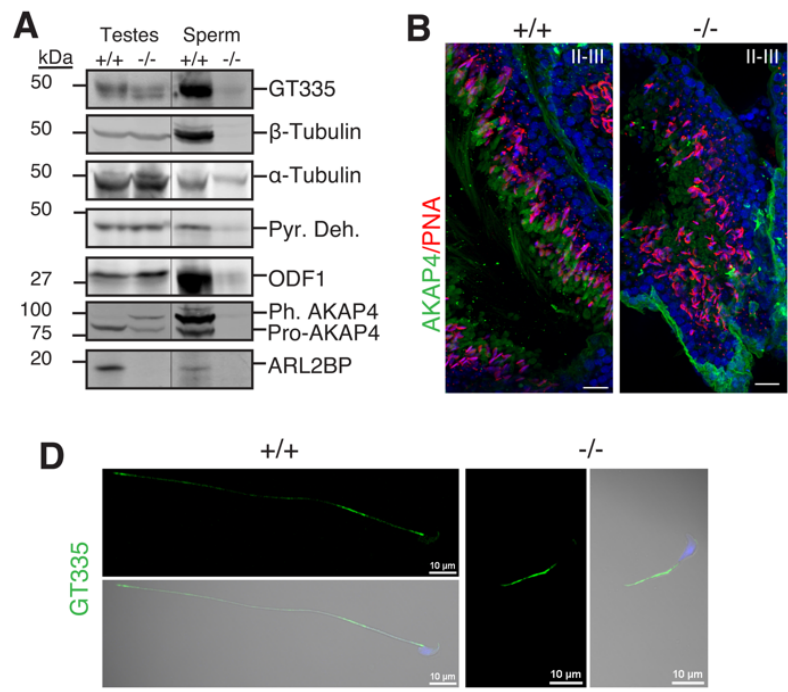

E
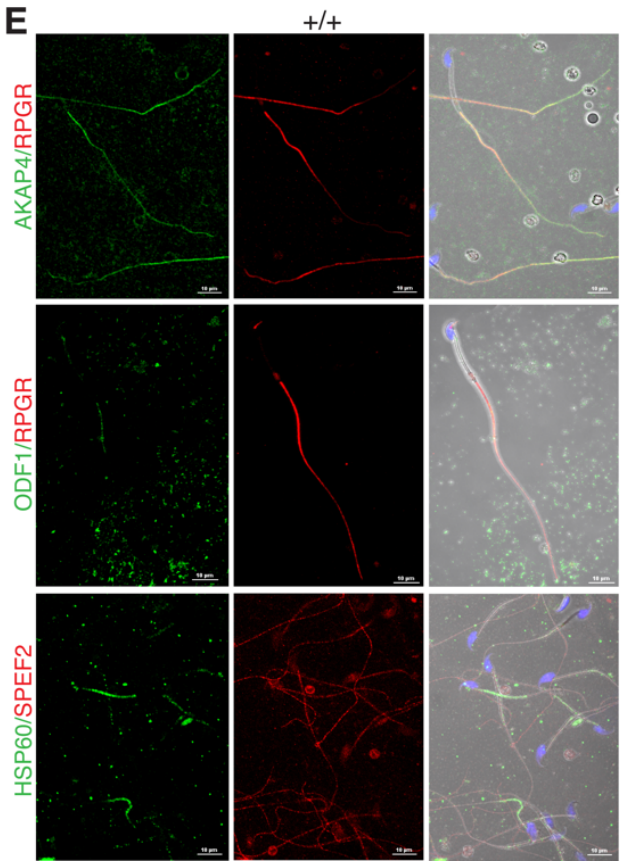

C

GT335/Mitotracker

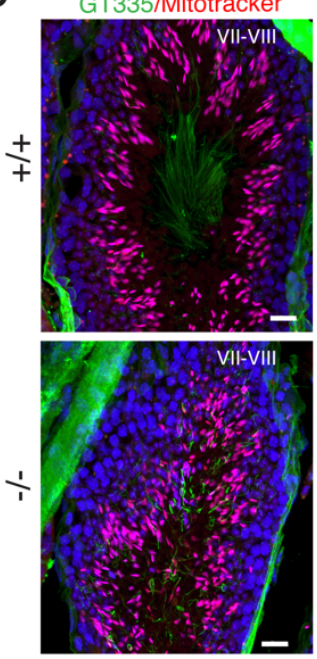

$-1-$
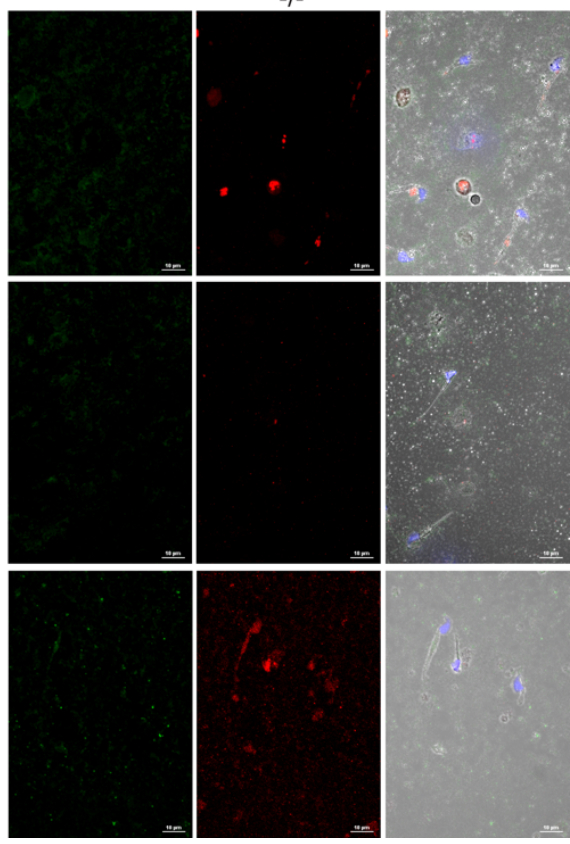

Figure 6. ARL2BP

loss results in impaired sperm tail development.

A. Immunoblot of WT $(+/+)$ and KO (-/-) testis and sperm lysates probed for the indicated sperm tail markers: axonemal markers (Glutamylated tubulin (GT335), Acetylated tubulin (Ac. Tubulin), $\beta$-tubulin, and $\alpha$-tubulin) and accessory structure markers (Pyruvate dehydrogenase (Pyr. Deh.), Outer Dense Fiber 1 (ODF1), and A-kinase anchoring protein 82 (preprocessed, phosphorylated $=$ ProAKAP4 and processed = AKAP4). Molecular weight displayed on the left. B. Graph displaying the normalized protein levels corresponding to

A). Pro-AKAP4

$\mathrm{p}=0.2004$, AKAP4 $\mathrm{p}=0.0068 . \mathrm{n}=3$. Data are represented as the mean \pm SEM. C and D. WT $(+/+)$ and $\mathrm{KO}(-/-)$ murine testes sections displaying staining of DAPI (nuclei, blue) and C) PNA lectin (acrosomes, red) and AKAP4 (green), or D) mitotracker (mitochondria, red) and GT335 (glutamylated tubulin, green). Scale bar $=20 \mu \mathrm{m}$. E and F. Sperm stained with the indicated sperm tail markers in WT (+/+) and KO (-/-) murine sperm. Glutamylated Tubulin, (GT335 axoneme, green), A-Kinase Associated Protein 4 (AKAP4 - fibrous sheath, green), Retinititis Pigmentosa GTPase Regulator (RPGR - axoneme, red), Outer Dense Fiber Protein 1, (ODF1 outer dense fibers, green), Heat Shock Protein 60kDa (HSP60 - mitochondrial sheath, green), and Sperm Flagellar Protein 2 (SPEF2 - axoneme, red). Scale bar $=10 \mu \mathrm{m}$. 


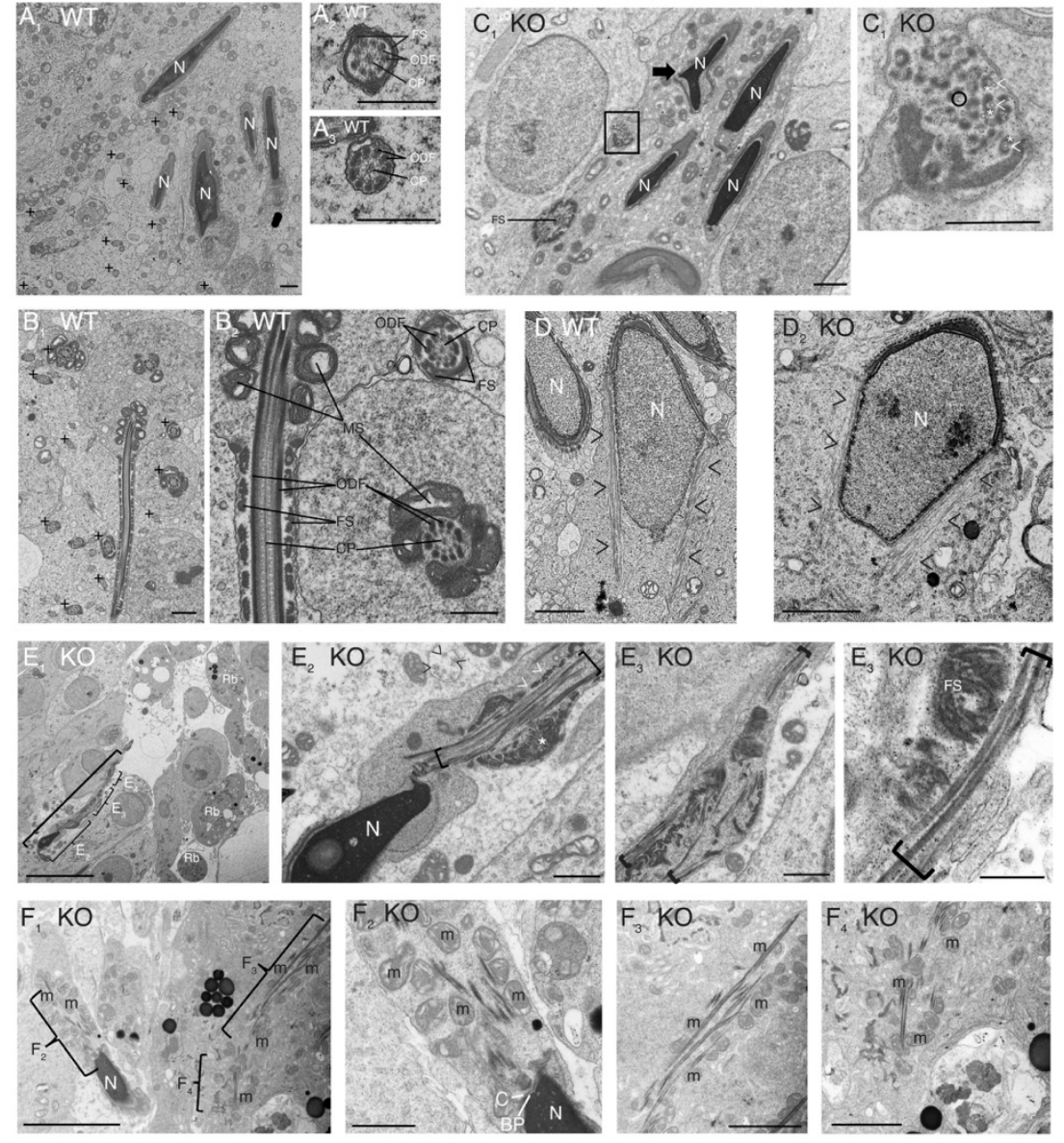

Figure 7. Loss of ARL2BP results in disorganized axoneme and accessory structures.

$\mathbf{A}_{1}-\mathbf{A}_{3}$. WT testes. $\mathbf{A}_{1}$. Multiple heads with normal nuclei $(\mathrm{N})$ and acrosomes. One acrosome appears somewhat abnormal but may be an artifact of preparation. Many ( $>20)$ tail crosssections are seen $(+)$. Bar $=1$ micron. $\mathbf{A}_{2}$ and $\mathbf{A}_{3}$ are tail cross-sections with the central pair (CP) microtubules of the $9+2$ axoneme, outer dense fibers (ODF), and fibrous sheath (FS). Bar $=500 \mathrm{~nm}$. $\mathbf{B}_{\mathbf{1}}$. WT testes show tail crosssections $(>20)$ with normal structures $(+)$. Bar $=1$ micron. $\mathbf{B}_{\mathbf{2}}$. Higher magnification of $\mathbf{B}_{\mathbf{1}}$. Bar $=$ $500 \mathrm{~nm} . \mathbf{C}_{\mathbf{1}}$. KO spermatid heads mostly normal with some abnormally shaped (arrow). Tail cross-sections appear to be disorganized FS components with disorganized microtubules (box). Bar $=1$ micron. $\mathbf{C}_{\mathbf{2}}$. Higher magnification of box in $\mathbf{C}_{\mathbf{1}}$ shows disorganized microtubules $(<)$ with associated ODF material $(*)$. Some microtubules are incomplete (circle). Bar $=500 \mathrm{~nm}$. D. Step 9 spermatids with normal manchettes $(><)$ in WT $\left(\mathbf{D}_{1}\right)$ and $\mathrm{KO}\left(\mathbf{D}_{2}\right)$. Bars $=1$ micron. $\mathbf{E}_{\mathbf{1}}$. Abnormal step 15 spermatid in $\mathrm{KO}$ (bracket) with microtubules, ODF, and FS present but disorganized (Higher mag in $\mathbf{E}_{\mathbf{2}}, \mathbf{E}_{\mathbf{3}}$ Bar $=1$ micron, and $\mathbf{E}_{\mathbf{4}}$ Bar $\left.=500 \mathrm{~nm}\right)$. Residual bodies $(\mathrm{Rb})$ present in the lumen. Bar $=10$ microns. $\mathbf{E}_{\mathbf{2}}$. Abnormal head and neck region with parallel microtubules and disorganized tail accessory structures. Microtubules (bracket), putative ODF $(>)$, and putative FS (*). Tail cross-section top/center with single, unorganized microtubules $(<)$. Bar $=1$ micron. $\mathbf{F}_{\mathbf{1}}-\mathbf{F}_{\mathbf{4}} \cdot \mathrm{KO}$ testes show disorganized microtubules and aggregation of mitochondria (M) that fail to organize into a tight spiral. Bar $=$ 5 microns; $\mathbf{F}_{\mathbf{3}}$ and $\mathbf{F}_{\mathbf{4}}$ Bar $=2$ microns. $\mathbf{F}_{\mathbf{2}} \mathbf{M}$ and microtubules are disorganized. In the neck region, basal plate $(\mathrm{BP})$ and capitulum $(\mathrm{C})$ are seen. $\mathrm{Bar}=1$ micron. 

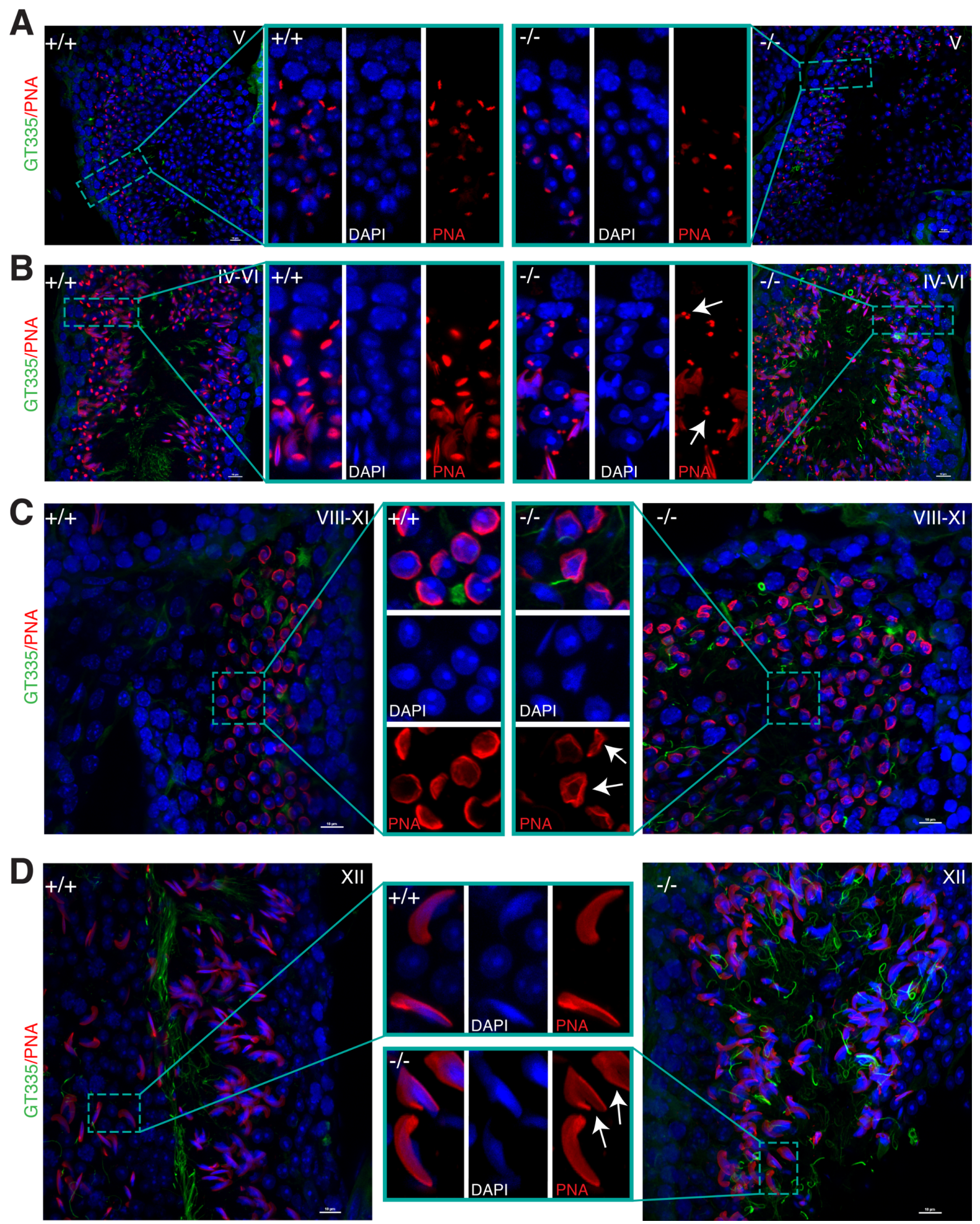

Figure 8. Abnormal acrosomes with loss of ARL2BP.

A-D. WT (+/+) and KO (-/-) adult murine testes sections displaying staining of DAPI (nuclei, blue), PNA lectin (acrosomes, red) and GT335 (green, sperm tails in stages IV-VI and XII). Scale $b a r=10 \mu \mathrm{m}$. Inserts show zoomed in sections from each image. Arrows point to abnormal acrosomes. 
A + + Situs Solitus

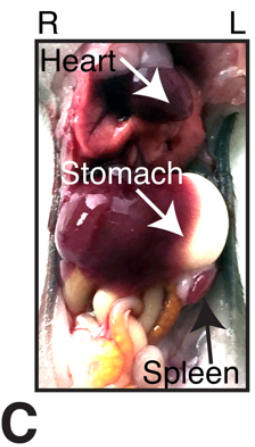

Situs Inversus
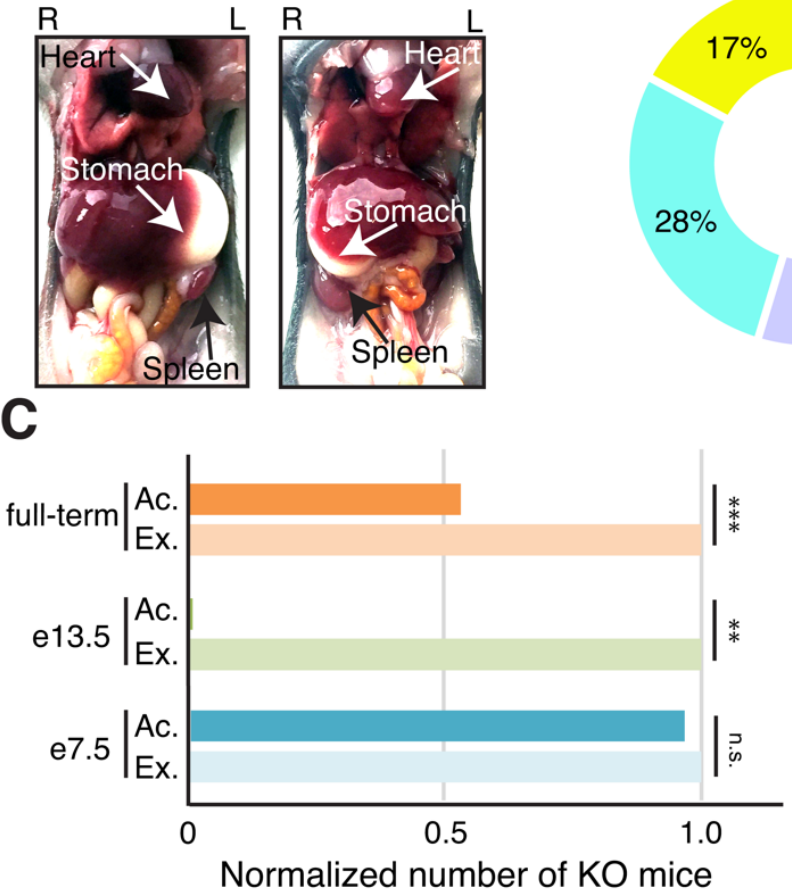

Normalized number of $\mathrm{KO}$ mice

E

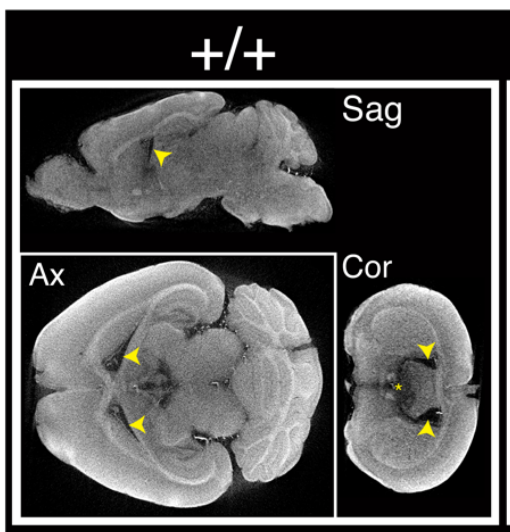

B

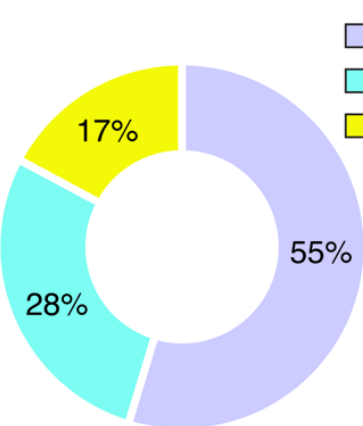

Situs Inversus

Heterotaxy

Situs Solitus

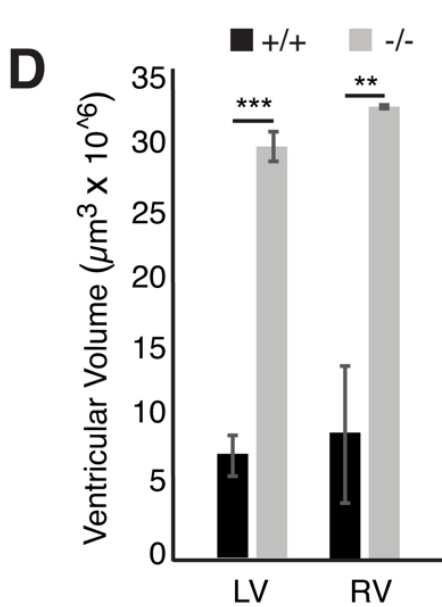

Figure 9.

Laterality defects and enlarged lateral ventricles in the adult murine brain with absence of ARL2BP.

A. WT $(+/+)$ mouse displaying situs solitus (normal axis patterning), and $\mathrm{KO}$ (-/-) mouse exhibiting situs inversus (complete reversal). B.

Percentages of KO animals with left/rightasymmetry abnormalities. C. Graph displaying the non-Mendelian distribution of $\mathrm{KO}$ animals identified from Het x KO crosses of full-term litters (total of 100 animals with 23 KO's, chi square value of 39.19 , $\mathrm{p}>0.001$ ), or collected at embryonic day 13.5 (total of 7 animals

with 0 KO's, chi square value of 7, 0.01>p >0.005), compared to the Mendelian distribution seen at embryonic day 7.5 (total of 24 animals with 16 KO's, chi square value of 0.96). D. Ventricular volume comparison between WT (+/+) and KO (-/-) brains from 3D-recontruction of CT images. $\mathrm{n}=3$. Left $\mathrm{p}=0.0002$, Right $\mathrm{p}=0.0087$. Data are represented as the mean $\pm \mathrm{SEM}$. E. Micro-CT scans of WT (+/+) and KO (-/-) murine brains at postnatal day 60, in Sagittal (Sag), Axial (Ax), and Coronal (Cor) planes. Arrows mark the enlarged lateral ventricles. Ac.=Actual \#, Ex.=Expected \#; LV=left ventricle, $\mathrm{RV}=$ right ventricle. 
A
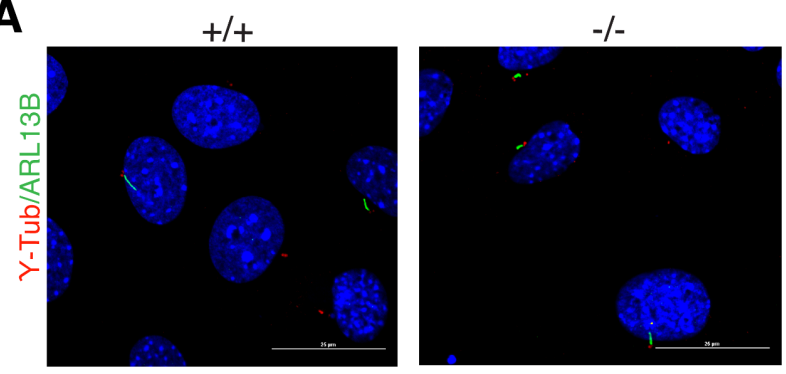

C

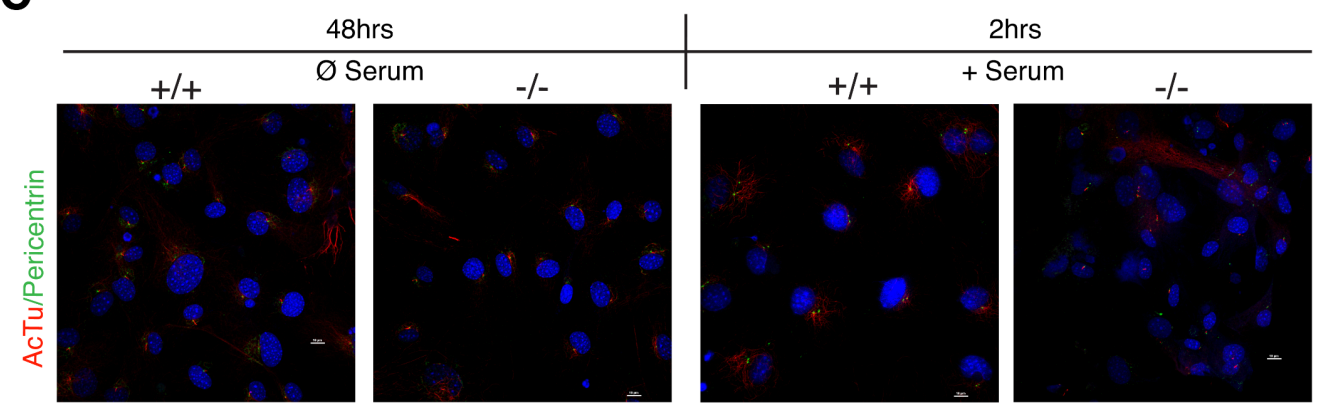

D

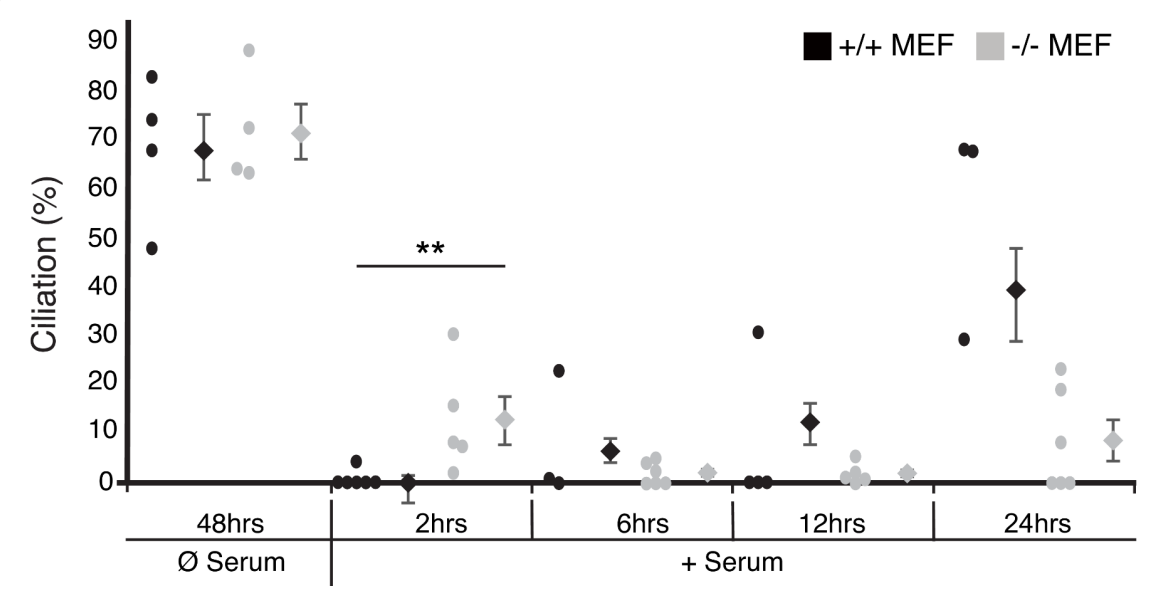

B

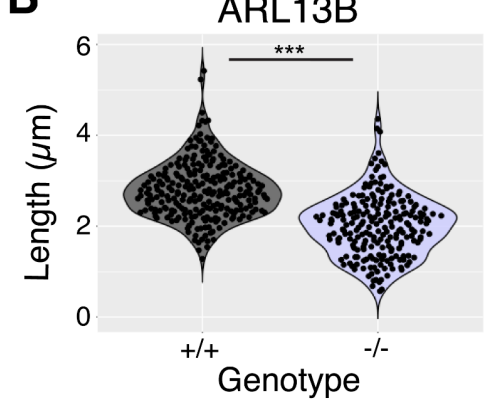

Figure 10. Cells lacking ARL2BP undergo cilia depolymerization more slowly.

A. Mouse embryonic fibroblasts (MEFs) from WT $(+/+)$ and $\mathrm{KO}(-/-)$ littermates stained to show DAPI (nuclei, blue), ARL13B (cilia, green), and $\gamma$-tubulin (basal body, red). B. Violin plot displaying the length measurements from WT $(+/+)$ and KO (-/-) MEFs. $\mathrm{n}=200 . \mathrm{P}=0.0001 \mathbf{C}$. MEFs from WT (+/+) and KO (-/-) littermates after serum starvation (cilia induction) for 48 hours, followed by serum addition (cilia depolymerization) for 2 hours stained for Acetylated tubulin (AcTu - cilia, red) and pericentrin (basal body, green). D. Dot plot displaying the percentage of ciliated cells between WT (+/+) and KO (-/-) MEFs after 48 hours of serum starvation and at indicated time points after re-addition of serum. Each dot represents $200 \geq 100$ cells. Average percentage of ciliated cells is to the right of each dot set. Data are represented as the mean \pm SEM. For $2 \mathrm{hrs}, \mathrm{p}=0.0174$. 
Table Titles and Legends

\section{Table 1.}

Spermiogram of patient P1

\begin{tabular}{lcc}
\hline & P1 & Lower reference limit $^{\mathrm{A}}$ \\
\hline Semen volume $(\mathrm{ml})$ & 1 & 1.5 \\
Sperm concentration $\left(10^{6} / \mathrm{ml}\right)$ & 50 & 15 \\
Total number $\left(10^{6} /\right.$ ejaculate $)$ & 50 & 39 \\
Total motility $(\%)$ & 0 & 40 \\
Normal forms (\%) & 8 & 4 \\
$\mathrm{pH}$ & 9 & 7.2 \\
\hline
\end{tabular}

${ }^{A}$ Reference data from WHO (PMID:19934213)

${ }^{B}$ Total motility includes progressive and non-progressive motility 


\section{Chapter 4: Discussion and Future Directions}

My studies on ARL2BP have proven that this small protein is required for cilia structure and function in the photoreceptors and sperm flagellum, and most likely for other cilia present throughout the body including the developing node and brain. ARL2BP was discovered due to its interaction with ARL2 in an "in vitro" binding assay or in exogenous overexpression cell culture systems. However, we have not been able to prove the interaction with ARL2 in photoreceptor or in sperm cells under native conditions. Furthermore, my findings suggest that defects with loss of ARL2BP are not realized through changes in function/localization of ARL2. However, I cannot rule out the possibility that this interaction is transient, and some of the symptoms observed in ARL2BP KO mice and patients could be related to ARL2 dysfunction. Nevertheless, it is clear that ARL2BP has independent, cilia-related functions. In this discussion, I hope to convince the reader that ARL2BP should be renamed Cilia and Flagella Associated Protein 20 Binding Partner (CFAP20BP), as its primary function is unrelated to ARL2. 


\section{ARL2BP in the realm of ciliopathies}

Mutations in ARL2BP cause many symptoms related to ciliopathies, such as RP, Situs Inversus, male infertility, as well as more variable symptoms like mild hydrocephalus, and respiratory symptoms (Davidson et al., 2013; Audo et al., 2016; Fiorentino et al., 2018; Moye et al., 2018; Chapter 3). These symptoms are most related to the ciliopathy Primary Cilia Dyskinesia (Leigh et al., 2009). Mutations causing PCD are usually associated with motile cilia (ex. DNAI1 , DNAH5 , DNAH11), but there is variability in phenotypes associated with primary cilia as well, such as RP (Leigh et al., 2009). Therefore, mutations in ARL2BP are most closely linked to PCD, which I term as "non-classical," since the most classical PCD symptom is respiratory related and has only been seen in one ARL2BP patient.

Mutations in Spef2 or CFAP221 (Pcdp1) in mice result in classical PCD symptoms, such as hydrocephaly, respiratory symptoms, and male infertility (Lee et al., 2008; Sironen et al., 2011; McKenzie et al., 2013). CFAP221 is a central pair protein that regulates ciliary motility through interactions with Calmodulin (DiPetrillo and Smith, 2010), and SPEF2 is a central pair protein whose exact function is unknown, but has been implicated in manchette function in spermatogenesis (Lehti et al., 2017). The severity of hydrocephalus in the mouse models for both of these proteins were found to be strain-dependent, worse in C57BL/6J than in $129 \mathrm{~S} 6 / \mathrm{SV}-\mathrm{E}$, and it is thought this variability is due to genetic factors unrelated to cilia (Finn et al., 2014; McKenzie et al., 2018). The ARL2BP mouse model was initially characterized in the C57BL/6J background, but lower numbers of KO pups pushed us to breed mice into the 129/SV-E background. This is the background in which the lateral ventricles of the brain were examined under, and is consistent with the published observation of less severe hydrocephalus in 129/SV-E backgrounds (Finn et al., 2014). Similarly, the SPEF2 and PCDP1 mouse models also showed 
variability in the severity of respiratory phenotypes (Lee et al., 2008; McKenzie et al., 2013). Furthermore, mouse models lacking either SPAG16 or SPAG6, both central pair proteins that interact on the axoneme, result in PCD phenotypes (hydrocephalus and male infertility) but neither reported any respiratory symptoms (Sapiro et al., 2002; Zhang et al., 2002; Zhang et al., 2006). However, a mouse model for SPAG16/SPAG6 displayed a severe pulmonary phenotype (Zhang et al., 2007). These findings point to the fact that PCD is a disease with variable phenotypic severity, especially in mouse models, where genetic backgrounds play a major role in the phenotypes observed. My studies on ARL2BP are consistent with previous work showing a severe phenotype in C57BL/6J in comparison to 129/SV-E background. With this finding in mind and the overall ciliary impact that loss of ARL2BP has, I propose that ARL2BP is a PCDassociated gene and renamed CFAP-Associated Protein.

One aspect to note throughout the ciliopathy research is that several genes identified cause a myriad of symptoms. The problem is that most labs are studying one protein in one tissue and do not have the capability to examine every possible cilia-related phenotype. For instance, there are a few mouse model descriptions that observed PCD-related symptoms, but the full array of ciliopathy phenotypes were not analyzed, such as retinal function. One way to bypass this is to document all possible cilia-related phenotypes in the animal model or patient being studied, and to create a database for the cilia field to start grouping genes according to symptoms and specific cilia dysfunctions (functional, structural, etc).

On that note, the main goal of my research was not only to document the symptoms related to mutations in, or loss of, ARL2BP, but to uncover its function, which I will discuss below. 


\section{ARL2BP does not interact with ARL2/ARL3}

ARL2BP was first discovered, and named, because of its interaction with ARL2. ADPRibosylation Factor Like protein 2 is a small GTPase that has dual roles in microtubule regulation and mitochondria. ARL2BP also bound, with less affinity, to ARL3, which has a very high homology to ARL2 (Sharer and Kahn, 1999). ARL2 and ARL2BP were also found to localize to centrosomes of dividing cells and to the IMS of mitochondria (Sharer et al., 2002; Zhou et al., 2006). Even though functional analysis did not implicate a role for ARL2BP in cell division or mitochondrial function, a crystal structure of the interaction between ARL2-GTP and ARL2BP was generated ((Zhang et al., 2009a) and scheme in Figure 1). Therefore, I investigated this interaction in vivo and viewed the symptoms in the ARL2BP KO mice with an ARL2colored lens. However, every attempt I made at proving this interaction failed (Figure 1). Briefly summarized in this discussion are the various approaches used to investigate the interaction of ARL2BP and ARL2 in photoreceptor cells and in testes.

1. Immunoprecipitation of native ARL2BP from testes lysates, followed by immunoblotting with polyclonal ARL2 antibody. As expected, I observed robust pull-down of ARL2BP (Figure 1B). However, ARL2 was not found in the eluted fraction. Tissue from ARL2BP knockout was used as negative control. Since it is possible that ARL2-ARL2BP could be promoted by GTP binding, this assay was performed in the presence of non-hydrolyzable GTP and was still unsuccessful in detecting this interaction. Similar results were obtained when retinal tissue was used (Figure 1C).

2. I performed a reciprocal IP with ARL2 and tried to detect ARL2BP interaction. In this case, I used the retinal tissue from transgenic animals expressing HA-tagged dominant-active 
ARL2 (Tg+). I could detect an interaction with TBCD and ARL2 but did not find ARL2BP

(Figure 1D). Littermate transgenic negatives were used as controls (Tg-).
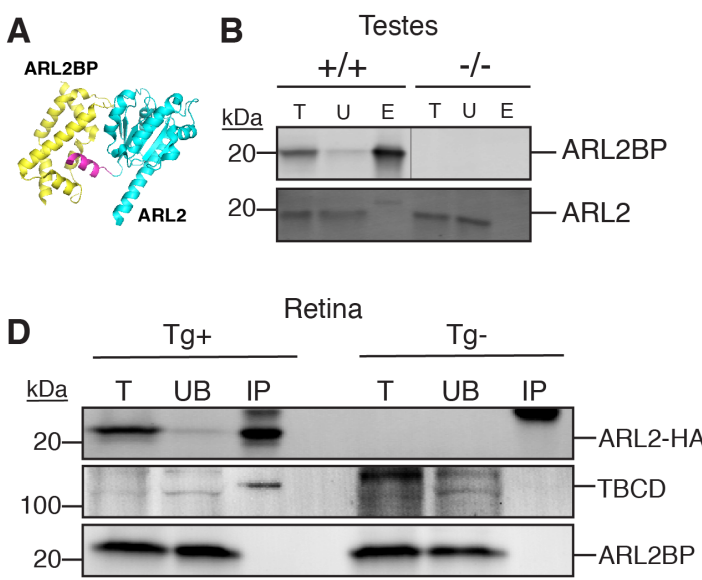

$\mathbf{F}$

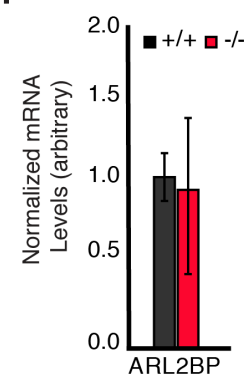

H

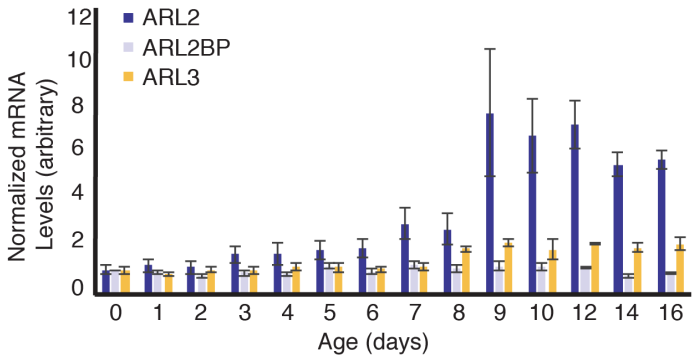

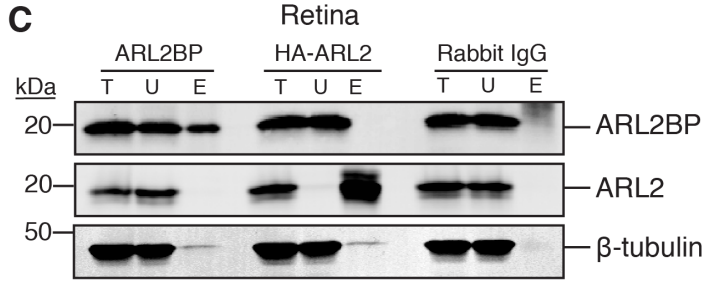

E
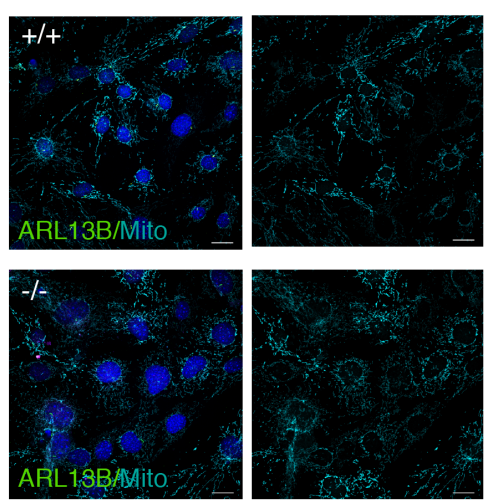

Figure 1. ARL2BP does not interact with ARL2

A. Cartoon structure of the published crystal structure of ARL2BP and ARL2-GTP interaction. ARL2BP is in yellow, ARL2 in cyan, and ARL2 $\mathrm{N}$-terminus in pink. B and C. IP from WT $(+/+)$ and $\mathrm{KO}(-/-)$ testis lysates $(\mathrm{B})$ or retinal lysates (C) ARL2BP antibody bound to agarose beads, with the $-/$ - serving as a negative control. Blots are probed with the antibodies indicated on the right. D. IP from WT retina (Tg-) or retina expressing ARL2 (Q70L)-HA $(\mathrm{Tg}+)$. HA-bound beads, and the $\mathrm{Tg}$ - is the negative control. E. ARL2BP WT (+/+) and KO (-/-) MEFs stained for cilia (ARL13B) and mitochondria. F and G. Relative mRNA levels of ARL2BP (F) and ARL2 (G) in retinal lysates. $\mathbf{H}$. Developmental time course of relative mRNA expression in retinal lysates of ARL2BP, ARL2, and ARL3 from postnatal day 0 (P0) - P16.

3. I compared the retinal phenotypes from mouse models for ARL2 and observed a few similarities with the ARL2-Q70L transgenic model, i.e., progression of retinopathy, and shortened cilia (Wright, et. al., accepted in Scientific Reports, 2018). However, ARL2 and ARL3 levels were normal in ARL2BP KO mice, and ARL2 mRNA levels were also unchanged (Chapter 2 Supplemental and Figure 1F and G). In addition, photoreceptor degeneration is rapid in ARL2 animals in comparison to ARL2BP animals. 
4. A developmental mRNA expression time-course revealed that the expression of ARL2BP in retina did not correlate with ARL2 (Figure 1H).

5. Since defects in ARL2 affect mitochondria, I examined the morphology of the mitochondria in TEM images of WT and ARL2BP KO photoreceptors, and found them to be comparable.

6. I then discovered that the ARL2BP KO mice had a defect in sperm, and that ARL2BP was expressed at higher levels in testes than in retina. Since sperm rely heavily upon proper mitochondrial localization and fusion, I thought that if ARL2BP does play a role with ARL2 in mitochondria, I would observe it in sperm. So, I again looked at mitochondrial morphology using TEM and found that the mitochondrial sheath was completely disrupted (Chapter 3, Figure 7), indicating a problem in mitochondrial motility. I also found that the outer dense fibers in the $\mathrm{KO}$ sperm were not properly localized, which could be due to a loss of fusion with the mitochondria (discussed in Chapter 3). It is difficult to interpret whether the mitochondrial defect seen is primary or secondary to the microtubule structural impairments. Many mouse models of mitochondria-related proteins show sperm phenotypes similar to the ARL2BP KO model. Aside from MNS1 discussed in Chapter 3, loss of the cation channel VDAC3 results in loss of 1 DMT, slow motility, and enlarged and slightly disorganized mitochondria (Sampson et al., 2001). However, there are many mouse models of non-mitochondrial proteins that display mitochondrial sheath disruptions as well. For instance, Galnt3-/- mice (an acrosome-related protein) had deformed acrosomes and slightly disturbed mitochondrial arrangement (Miyazaki et al., 2013), and similarly, loss of Iqcg in a mouse model resulted in shortened sperm tails with misshapen heads and slightly disrupted mitochondrial arrangement (Li et al., 2014). With these 
results in mind, I could not conclude whether the sperm phenotype in ARL2BP KO mice is related to ARL2.

Therefore, I took advantage of the mouse embryonic fibroblasts I had generated from WT and ARL2BP KO mice to explore this interaction as well. I first confirmed that primary cilia induced in these cells had a phenotype (Chapter 3, Figure 10). Then I stained for mitochondria, cilia, and ARL2 to examine mitochondrial morphology and ARL2 localization in ciliated cells (important, considering that the majority of the research being done on ARL2 in other labs is not in ciliated cells). After I performed this staining, a paper was published on ARL2 discussing the difficulty in ARL2 localization to mitochondria in cells and explaining that the staining is very sensitive to any cell manipulation. This, combined with the low levels present in the MEFs (data not shown), might explain why the ARL2 staining did not seem to work. However, I was able to see mitochondria clearly through immunofluorescence and found that there was no noticeable fragmentation or tubulation in ARL2BP KO MEFs (Figure 1E).

7. Lastly, we tried an unbiased approach to test the interaction with ARL2 and ARL2BP, by performing immunoprecipitation paired with mass spectrometry analysis. Daniella Munezero, my colleague, used retinal lysates from WT and ARL2BP KO, then I independently used testes lysates from WT and ARL2BP KO for ARL2BP pull-downs. Interacting partners, including ARL2BP bound to column, were eluted by SDS-PAGE buffer and sent to an outside company for gel analyses followed by mass-spectrometry. Not surprisingly, neither ARL2 nor ARL3 were present in IP samples. Therefore, I pursued the possibility of identifying novel interactors of ARL2BP. 
In summary, our investigations do not show an interaction of ARL2BP with ARL2. It is possible that this interaction is transient and/or the native levels in vivo are low, making this interaction difficult to capture. Alternatively, since previous studies were performed using overexpressed, recombinant proteins, ARL2BP is likely not a binding partner of ARL2.

\section{Novel interactors of ARL2BP}

To investigate the role for ARL2BP outside of ARL2, I first analyzed the mass spectrometry results for clues. I compared the proteins present in both WT samples that were not present in the KO samples, which produced 6 proteins (Figure 3A). Next, I used stringent criteria and ruled out proteins that had less than 5 hits in the WT and included proteins that had a 3 -fold or more increase between WT and KO. This left me with 2 proteins - Cul9, a ring E3 ubiquitin ligase of around $210 \mathrm{kDa}$, and cilia and flagella associated protein 20 (CFAP20), a small protein previously localized to the inner junction of microtubule doublets in Chlamydamonas. I then tested these interactions by immunoblotting, which showed that Cul9 came down in both WT and $\mathrm{KO}$, which is not entirely surprising as its large size would account for the increased number of hits, and is therefore likely not an interactor. Wonderfully though, CFAP20 came down quite strongly in the WT pull-downs! I then stained for CFAP20 in fixed and unfixed (cilia-specific) retinal sections (staining did not work well in MEFs or sperm), and found that there is an apparent increase in CFAP20 immunofluorescent staining in ARL2BP KO photoreceptor cilia. However, immunoblot analysis showed that CFAP20 levels were unchanged between WT and KO retina (Figure 3) (though this needs repeated in testes). This discrepancy could be attributed to the open IJ in ARL2BP KO cells resulting in an exposed epitope on CFAP20, resulting in an artificial increase of CFAP20 staining. 
Interestingly, CFAP20 (FAP20 in Chlamydamonas, and Bug22 in Drosophila) has not been investigated in mammalian cilia. However, the role for CFAP20 has been characterized in Drosophila, Zebrafish, Chlamydomonas, and other unicellular organisms. Use of Chlamydomonas was very helpful in determining the localization of FAP20 in the axoneme. FAP20 localized along the entire flagellar axoneme length. Additionally, in FAP20-/- all three beak-like structures (only present in Chlamydomonas B-tubules of DMT 1, 5, and 6) were missing (Yanagisawa et al., 2014), which was also shown to affect the planar beating (forwardmoving, cilia waveform) (Meng et al., 2014). In multi-cellular organisms, Bug22 is necessary for Drosophila sperm individualization and motility, but not for axoneme structure (Mendes Maia et al., 2014). Furthermore, Bug22 was localized to the nucleus, cilia, and sperm flagella in Drosophila. Another interesting discovery with the Bug22-/- was that polyglycylation of tubulin was reduced in mutant sperm. Upon further investigation of this phenotype, knockdown of CFAP20 in RPE1 cells caused a similar decrease in polyglycylation, with a corresponding increase in polyglutamylation. Furthermore, the cilia in CFAP20 KD cells were longer than in WT, as well as curved. Overall, Bug22 is important for cilia maintenance and PTM processing, but not for ciliogenesis in Chlamydomonas and Drosophila. This contradicts the studies in zebrafish, showing that morpholino KD of GTL3 (CFAP20) results in shorter and less cilia in Kupffers Vesicle (corresponds to the embryonic node), as well as other ciliary symptoms (curved body axis, and defective heart-looping orientation) (Yanagisawa et al., 2014). This discrepancy in phenotypes could be because this vertebrate model illustrates the need for ARL2BP (expressed only in vertebrates), and the varying role for CFAP20 in vertebrate ciliary function. PACRG and Tektin also fractionated with FAP20 in the sarkosyl detergent gradient, an experiment which is used to determine with what part of the DMTs a protein is associated. This 
result, and the fact that PACRG and Tektin levels were decreased in the FAP-/-

Chlamydomonas, suggested that they might also be IJ proteins (Yanagisawa et al., 2014). This is

A

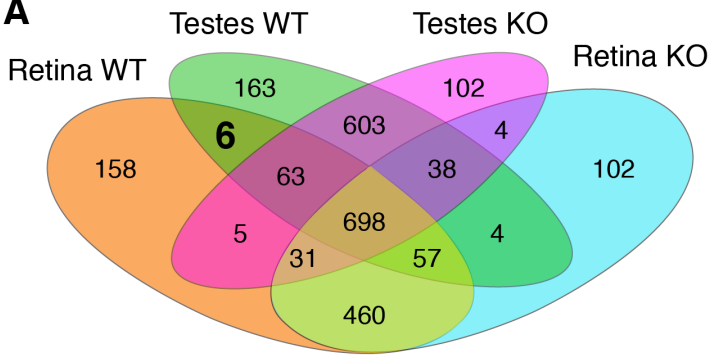

B

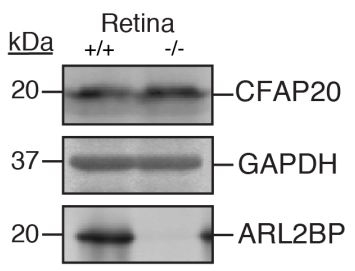

C

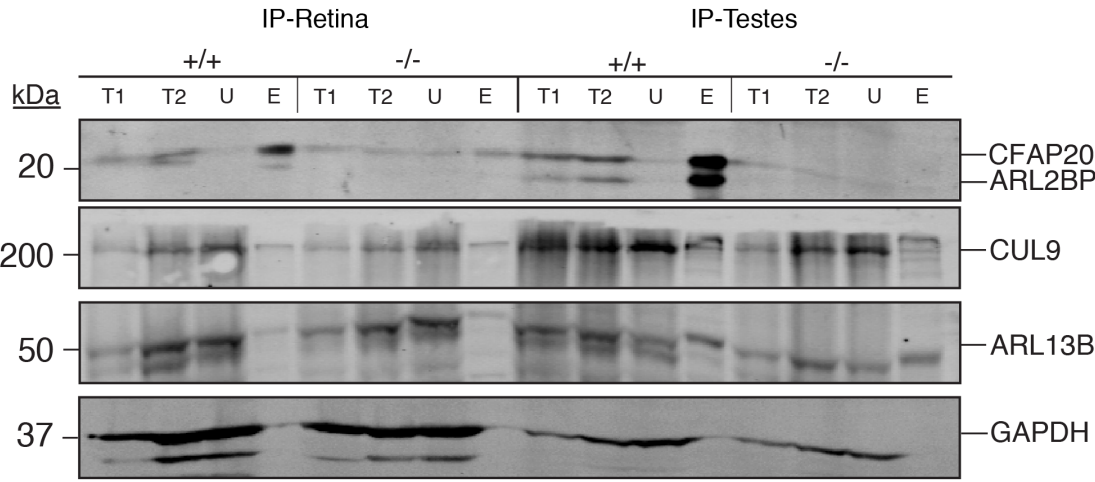

D
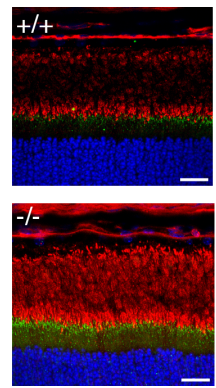

Fixed
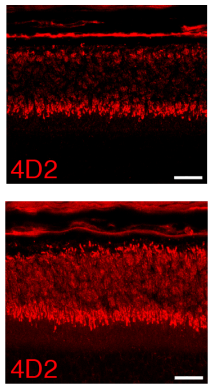

$\mathbf{E}$
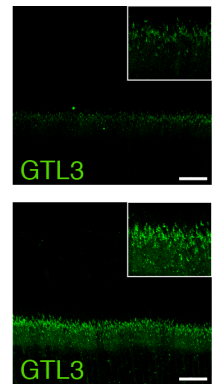
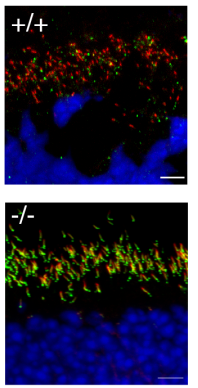

Unfixed
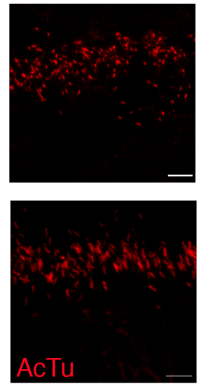
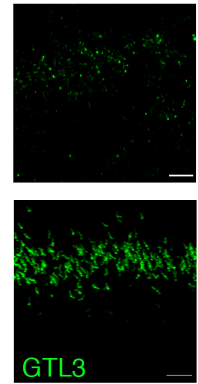

Figure 2. ARL2BP interaction with CFAP20

A. Venn Diagram illustrating the distribution of proteins pulled down in the mass spectrometry from WT and ARL2BP retina and testes. The number of proteins found only in the WT samples (6) are bolded. B. Immunoblot analysis from WT (+/+) and $\mathrm{KO}(-/)$ retina at P16. Antibodies indicated on the right. C. IP from WT (+/+) or ARL2BP KO (-/) retina and testis lysates. ARL2BP-bound beads, and the $-/$ - is the negative control. Showing that CFAP20 pulls down strongly in the WT samples, but that Cul9 and ARL13B pull down a little bit in both WT and KO samples. D and E. Retinal cross-sections from WT (+/+) and KO (-/-) animals at P16 stained for the indicated markers. D) normally fixed eyecups, scale bar $=20 \mu \mathrm{m}$, E) unfixed eyecups, used for the staining of ciliary proteins, scale bar $=5 \mu \mathrm{m} .+/+$ sample was partially washed away, so the imaging is fuzzier than it should be, but $n=3$ still needs to be completed.

interesting, as PACRG has also been shown to be required for male spermiogenesis (Lorenzetti

et al., 2004; Yanagimachi et al., 2004). Similarly, an interactor of PACRG, MEIG1, was also

found to be required for spermiogenesis, with abnormal tail formation and disrupted manchette 
seen in KO mice (Zhang et al., 2009b; Salzberg et al., 2010; Li et al., 2015). It was proposed that PACRG and MEIG1 are required for intra-manchette transport during spermiogenesis, and loss of IMT results in disruption of the 9+2 axoneme structure and proper assembly of periaxonemal structures. Since these phenotypes are similar to those seen in ARL2BP KO sperm, this introduces the thought that ARL2BP interaction with CFAP20 could resemble MEIG1 interaction with PACRG.

It is most probable that with loss of ARL2BP, CFAP20 is mislocalized from the IJ, and loss of the proper localization of both of these affects the localization of additional stabilizing structures, such as MEIG1 or tektin proteins. This would result in an open IJ in photoreceptor cilia and unstable B-tubules. However, without all the affected proteins identified, or a mammalian model for CFAP, this does not clearly explain the severity of phenotypes seen in photoreceptor cells or sperm. For instance, RPGR is mislocalized, or possibly decreased, in both
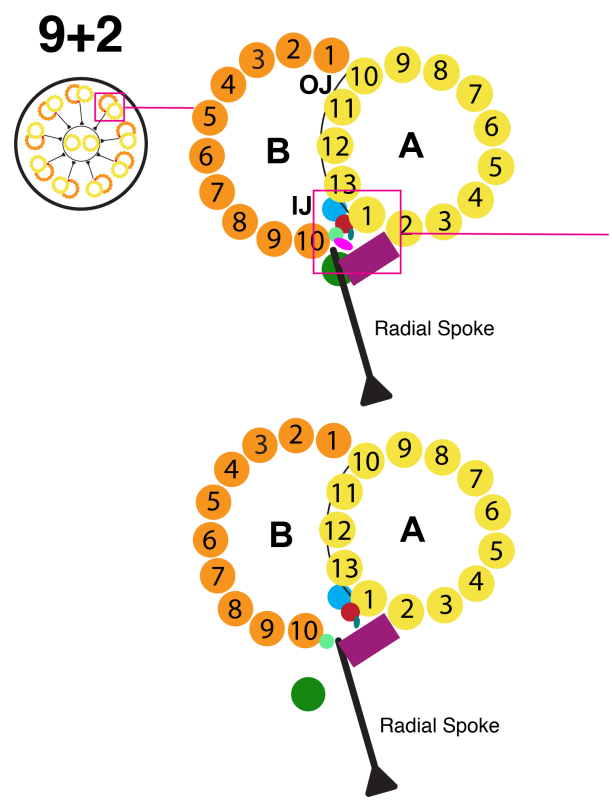
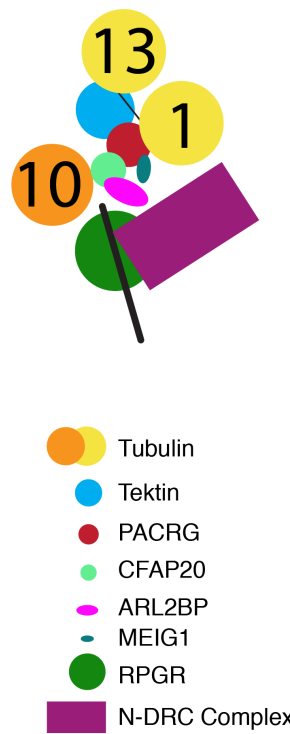

Figure 3a. Model for the role of ARL2BP.

9+2 cilia cross-section structure. WT on top. Without ARL2BP (bottom), The DMT might be less stable, and RPGR may mislocalize, but the IJ may be held in place by components of the radial spoke and N-DRC complex, which are not present in immotile cilia. photoreceptors and sperm of ARL2BP

KO mice. It is interesting to speculate that loss of ARL2BP may affect RPGR

through its interaction with CFAP20 and subsequent loss of structural complexes required for proper axonemal assembly.

This could also explain why loss of either RGPR or CFAP20 alone results in less severe phenotypes than loss of ARL2BP (Md et al., 1997; Hosch et al., 2011). The interaction between ARL2BP 
and CFAP20 also does not explain why loss of ARL13B in nodal cilia results in an open IJ.

Though, the roles for ARL13B are so diverse, it is likely a completely unrelated side-effect. And, importantly, we also confirmed that ARL2BP and ARL13B do not interact (Figure 2C).

One interesting point to mention is that the cilia affected with loss of ARL2BP are all monocilia, while the multi-cilia seem to have more of an ability to compensate. It is perhaps because the cilia present on multi-ciliated cells are more similar to their invertebrate ancestral cilia, and therefore loss in localization of CFAP20 is not as detrimental to their function. Furthermore, non-motile cilia lack structural protein complexes present in

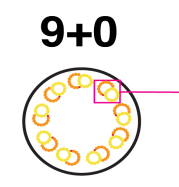
the motile cilia, such as the DRC complex (Ishikawa et al., 2012). This could explain the more severe susceptibility to loss of ARL2BP, and CFAP20 disruption, in photoreceptors and in the nodal cilia as compared to the brain (Figure 3a). I

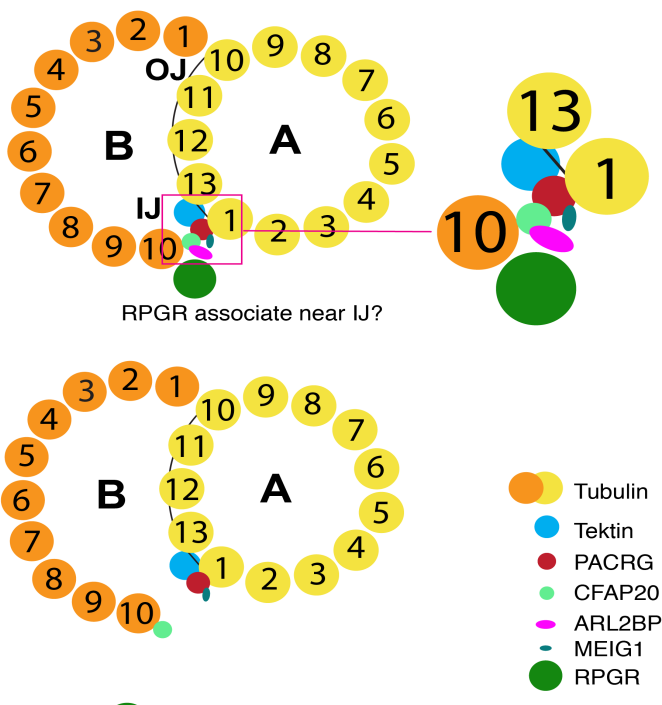

Figure 3b. Model for the role of ARL2BP. $9+\mathbf{0}$ cilia cross-section structure. WT on top. ARL2BP interacts with CFAP20, an inner junction (IJ) protein. Without ARL2BP (bottom), IJ is open, CFAP20 is exposed, and RPGR is mislocalized.

hypothesize that the relatively severe disruption in spermiogenesis is due to the need for proper manchette and axoneme flagellum assembly before they are even motile.

The original role for ARL2BP was as an effector for ARL2-GTP and ciliogenesis. Based on my results from published and unpublished data, I have identified ARL2BP as being essential for cilia microtubule structure. With results from the studies I performed on retina, I was able to identify the need for ARL2BP in DMT formation, but at that stage did not know if ARL2BP was 
itself an IJ protein, or was required for localization of IJ proteins. My studies on sperm flagellum and MEFs added results confirming the need for ARL2BP in cilia length maintenance and microtubule structure, but still did not answer my initial questions. Additional, as yet unpublished, work has provided these answers: that ARL2BP is not an IJ protein, or MT-binding protein. I have subsequently proved that ARL2BP interacts with CFAP20, a known IJ protein. I hypothesize, based on retinal ciliary staining observations, that ARL2BP serves as a specialized chaperone for CFAP20 in vertebrate species, maintaining its localization to the IJ all along the axoneme. Disruption in this interaction (like loss of ARL2BP) affects the structural integrity of the IJ and associated axonemal proteins, like that of RPGR (Figure 3b). Though the interaction between ARL2BP and CFAP20 is a promising next step in identifying the exact role for ARL2BP and its mechanism of action, there is still much work to be done (Figure 3c).

\section{Future Investigations}

To further understand the role of ARL2BP, it would first need to be proven that disruption of the interaction between CFAP20 and ARL2BP causes the phenotypes observed in ARL2BP KO mice. Retina or MEFs would be the preferred experimental system. For in vitro studies, WT and ARL2BP KO MEFs could be used to show CFAP20 localization. Then recombinant HA-

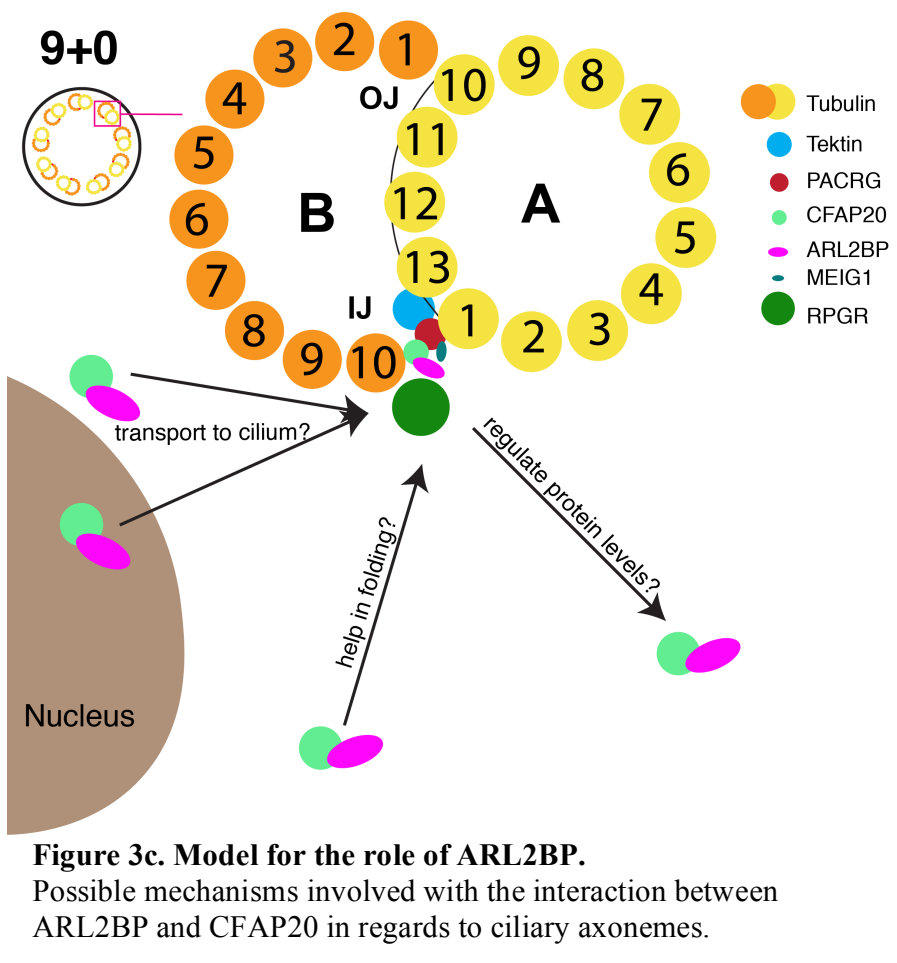


tagged ARL2BP could be expressed to rescue the cilia phenotype and see if CFAP20 localization is also rescued. Contrarily, it is possible that CFAP20 is upregulated in ARL2BP KO mice, which causes shortening of the cilia. This correlates with the study demonstrating longer cilia with KD of CFAP20 in RPE1 cells. In this case, knocking down CFAP20 in ARL2BP KO MEFs should also rescue the phenotype. In addition, functional studies will need to be performed. For this, the tractable ARL2BP KO MEFs (and generate CFAP20 MEFs) could be used to introduce patient mutations of ARL2BP. This would allow for the analysis of these patient mutations on the interaction of ARL2BP with CFAP20. This would also allow for the identification of the important domains of each protein, providing insight into ARL2BP's mode of action.

Comparatively, for in vivo analysis, sub-retinal injections of HA-tagged ARL2BP-AAV could be performed. If done before photoreceptor cell degeneration is triggered (P10), rescue of photoreceptor cilia shortening and OS dysmorphia could be seen, as well as examine CFAP20 localization (and other related proteins). Finally, a CFAP20 KO model needs to be made to directly compare the phenotypes. This model could be used to analyze the protein levels and localization of ARL2BP, and other related proteins like that of PACRG, tektin, MEIG1, and RPGR, though as this is much more time and labor intensive, and the other experiments should be completed first.

Additionally, more structural analysis between WT, ARL2BP KO, and maybe CFAP20 KO (in a vertebrate model) cilia could be done as well. First, it would be beneficial to cross the ARL2BP (or CFAP20) mice with SSTR3-GFP mice (fluorescently labels cilia). Then, to obtain cross-sectional views of $\mathrm{CC}$ in photoreceptors, the eyecup could be rotated during cryosectioning instead of staying in plane with the laminar segments of the retina (Figure 4C). Structured Illumination Microscopy (SIM) imaging could then be used on the sections to achieve a more 
detailed analysis of the ciliary localization of ARL2BP or CFAP20. For now, we know ARL2BP does localize throughout the cilia in PR cells and sperm (Chapter 2 and 3). However, I found that

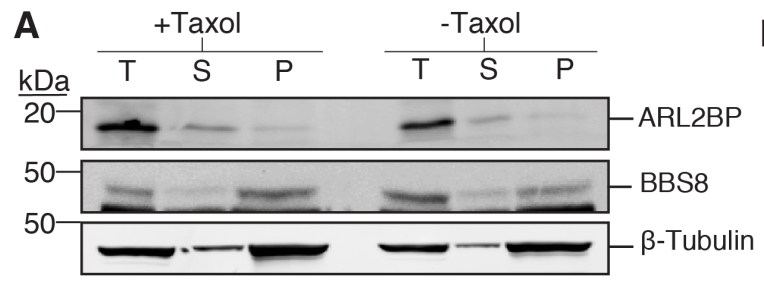

B

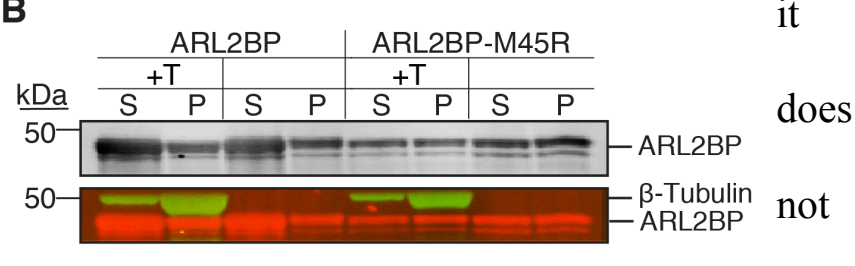

C

Laminated Retinal Layers

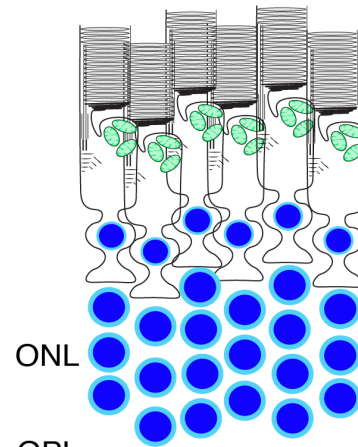

OPL

INL

IPL

$\mathrm{GCL}$
Flip on its side

Retinal Cilia cross sections

bind

to

microtubules, either with isolation of retinal polymerized microtubules, or using purified

recombinant proteins (Figure 4A and B). Therefore, this experiment would be very beneficial in deducing its exact role in regards to CFAP20, which does bind microtubules (Yanagisawa et al., 2014). Cryo-EM could then be performed on isolated sperm flagellum to analyze DMT structure and CFAP20 localization between WT, ARL2BP KO, and CFAP20 KO. Sperm flagellum seem to be the easiest cilia to isolate for cryo-EM, and more similar to Chlamydomonas flagellum compared to photoreceptor cilia. Also, CFAP20 has already been described using Cryo-EM in Chlamydomonas, so there is a guide of what to look for. In conjunction, biotin-tagged FAP20 and ARL2BP could be used for these Cryo-EM studies, with subsequent streptavidin labeling to 
identify the exact localization of these proteins in WT axonemes compared to the related KO's. Knowing the exact location of ARL2BP in reference to the DMTs will be exceedingly helpful in determining its function. 


\section{Conclusions}

Overall, these findings on ARL2BP presemt an important role for this protein in cilia structure. Specifically, I show that ARL2BP is required for photoreceptor cell and sperm function, as loss of ARL2BP results in open doublet microtubules in photoreceptor cilia, and unstructured microtubules in sperm flagellum. Although, in vitro studies identified ARL2BP as an interactor of ARL2, our studies show an ARL2-independent role for ARL2BP in the cilum. More importantly, we have uncovered an interaction between ARL2BP and CFAP20, a known structural element of cilia. This finding has provided a new path to understand the need for ARL2BP in ciliary function. Until now, most structural studies have been conducted using cilia from Chlamydomonas, especially considering that the non-tubulin structural proteins have only been identified relatively recently. Furthermore, the only other reported mouse model to possess open inner junctions, or an architectural defect in immotile cilia, was an Arl13b mutant, the study of which is complex due to embryonic lethality and severity in phenotypes. Therefore, the ARL2BP mouse model serves as an instrumental mammalian model system for the study of cilia. Importantly, research on ARL2BP has proven fruitful in identifying novel roles for non-MT binding proteins in regulation of cilia structure and stability. 


\section{References}

Audo, I., El Shamieh, S., Méjécase, C., Michiels, C., Demontant, V., Antonio, A., Condroyer, C., Boyard, F., Letexier, M., Saraiva, J.P., Blanchard, S., Mohand-Saïd, S., Sahel, J.A., and Zeitz, C. (2016). ARL2BP mutations account for $0.1 \%$ of autosomal recessive rod-cone dystrophies with the report of a novel splice variant. Clinical Genetics 92, 109-111.

Davidson, A.E., Schwarz, N., Zelinger, L., Stern-Schneider, G., Shoemark, A., Spitzbarth, B., Gross, M., Laxer, U., Sosna, J., Sergouniotis, P.I., Waseem, N.H., Wilson, R., Kahn, R.A., Plagnol, V., Wolfrum, U., Banin, E., Hardcastle, A.J., Cheetham, M.E., Sharon, D., and Webster, A.R. (2013). Mutations in ARL2BP, encoding ADP-ribosylation-factor-like 2 binding protein, cause autosomal-recessive retinitis pigmentosa. Am J Hum Genet 93, 321-329.

DiPetrillo, C.G., and Smith, E.F. (2010). Pcdp1 is a central apparatus protein that binds $\mathrm{Ca}(2+)-$ calmodulin and regulates ciliary motility. The Journal of cell biology 189, 601-612.

Finn, R., Evans, C.C., and Lee, L. (2014). Strain-Dependent Brain Defects in Mouse Models of Primary Ciliary Dyskinesia with Mutations in Pcdp1 and Spef2. Neuroscience 277, 552-567.

Fiorentino, A., Yu, J., Arno, G., Pontikos, N., Halford, S., Broadgate, S., Michaelides, M., Carss, K.J., Raymond, F.L., Cheetham, M.E., Webster, A.R., Downes, S.M., Hardcastle, A.J., for the, N.-B.R.D.C., and the, U.K.I.R.D.C. (2018). Novel homozygous splicing mutations in ARL2BP cause autosomal recessive retinitis pigmentosa. Molecular Vision 24, 603-612.

Hosch, J., Lorenz, B., and Stieger, K. (2011). RPGR: role in the photoreceptor cilium, human retinal disease, and gene therapy. Ophthalmic Genet 32, 1-11.

Ishikawa, H., Thompson, J., Yates, J.R., 3rd, and Marshall, W.F. (2012). Proteomic analysis of mammalian primary cilia. Current biology : CB 22, 414-419.

Lee, L., Campagna, D.R., Pinkus, J.L., Mulhern, H., Wyatt, T.A., Sisson, J.H., Pavlik, J.A., Pinkus, G.S., and Fleming, M.D. (2008). Primary ciliary dyskinesia in mice lacking the novel ciliary protein Pcdp1. Molecular and cellular biology 28, 949-957.

Lehti, M.S., Zhang, F.-P., Kotaja, N., and Sironen, A. (2017). SPEF2 functions in microtubulemediated transport in elongating spermatids to ensure proper male germ cell differentiation. Development 144, 2683-2693.

Leigh, M.W., Pittman, J.E., Carson, J.L., Ferkol, T.W., Dell, S.D., Davis, S.D., Knowles, M.R., and Zariwala, M.A. (2009). Clinical and Genetic Aspects of Primary Ciliary Dyskinesia / Kartagener Syndrome. Genetics in medicine : official journal of the American College of Medical Genetics 11, 473-487.

Li, R.-K., Tan, J.-L., Chen, L.-T., Feng, J.-S., Liang, W.-X., Guo, X.-J., Liu, P., Chen, Z., Sha, J.-H., Wang, Y.-F., and Chen, S.-J. (2014). Iqcg Is Essential for Sperm Flagellum Formation in Mice. PLoS ONE 9, e98053. 
Li, W., Tang, W., Teves, M.E., Zhang, Z., Zhang, L., Li, H., Archer, K.J., Peterson, D.L., Williams, D.C., Jr., Strauss, J.F., 3rd, and Zhang, Z. (2015). A MEIG1/PACRG complex in the manchette is essential for building the sperm flagella. Development (Cambridge, England) 142, 921-930.

Lorenzetti, D., Bishop, C.E., and Justice, M.J. (2004). Deletion of the Parkin coregulated gene causes male sterility in the quaking(viable) mouse mutant. Proceedings of the National Academy of Sciences of the United States of America 101, 8402-8407.

McKenzie, C.W., Klonoski, J.M., Maier, T., Trujillo, G., Vitiello, P.F., Huber, V.C., and Lee, L. (2013). Enhanced response to pulmonary Streptococcus pneumoniae infection is associated with primary ciliary dyskinesia in mice lacking Pcdp1 and Spef2. Cilia 2, 18-18.

McKenzie, C.W., Preston, C.C., Finn, R., Eyster, K.M., Faustino, R.S., and Lee, L. (2018). Strain-specific differences in brain gene expression in a hydrocephalic mouse model with motile cilia dysfunction. Scientific Reports 8, 13370.

Md, S.A., Ponjavic, V., Abrahamson, M., Ehinger, B., Wu, W., Fujita, R., Buraczynska, M., and Swaroop, A. (1997). Phenotypes in Three Swedish Families With X-linked Retinitis Pigmentosa Caused by Different Mutations in the RPGR Gene. American Journal of Ophthalmology 124, 95102.

Mendes Maia, T., Gogendeau, D., Pennetier, C., Janke, C., and Basto, R. (2014). Bug22 influences cilium morphology and the post-translational modification of ciliary microtubules. Biology open 3, 138-151.

Meng, D., Cao, M., Oda, T., and Pan, J. (2014). The conserved ciliary protein Bug22 controls planar beating of Chlamydomonas flagella. Journal of Cell Science 127, 281.

Miyazaki, T., Mori, M., Yoshida, C.A., Ito, C., Yamatoya, K., Moriishi, T., Kawai, Y., Komori, H., Kawane, T., Izumi, S.-i., Toshimori, K., and Komori, T. (2013). Galnt3 deficiency disrupts acrosome formation and leads to oligoasthenoteratozoospermia. Histochemistry and Cell Biology $139,339-354$.

Moye, A.R., Singh, R., Kimler, V.A., Dilan, T.L., Munezero, D., Saravanan, T., Goldberg, A.F.X., and Ramamurthy, V. (2018). ARL2BP, a protein linked to retinitis pigmentosa, is needed for normal photoreceptor cilia doublets and outer segment structure. Mol Biol Cell 29, 15901598 .

Salzberg, Y., Eldar, T., Karminsky, O.-D., Itach, S.B.-S., Pietrokovski, S., and Don, J. (2010). Meig1 deficiency causes a severe defect in mouse spermatogenesis. Developmental Biology 338, 158-167.

Sampson, M.J., Decker, W.K., Beaudet, A.L., Ruitenbeek, W., Armstrong, D., Hicks, M.J., and Craigen, W.J. (2001). Immotile sperm and infertility in mice lacking mitochondrial voltagedependent anion channel type 3. J Biol Chem 276, 39206-39212. 
Sapiro, R., Kostetskii, I., Olds-Clarke, P., Gerton, G.L., Radice, G.L., and Strauss, I.J. (2002). Male infertility, impaired sperm motility, and hydrocephalus in mice deficient in spermassociated antigen 6. Mol Cell Biol 22, 6298-6305.

Sharer, J.D., and Kahn, R.A. (1999). The ARF-like 2 (ARL2)-binding protein, BART. Purification, cloning, and initial characterization. J Biol Chem 274, 27553-27561.

Sharer, J.D., Shern, J.F., Van Valkenburgh, H., Wallace, D.C., and Kahn, R.A. (2002). ARL2 and BART enter mitochondria and bind the adenine nucleotide transporter. Mol Biol Cell 13, 7183.

Sironen, A., Kotaja, N., Mulhern, H., Wyatt, T.A., Sisson, J.H., Pavlik, J.A., Miiluniemi, M., Fleming, M.D., and Lee, L. (2011). Loss of SPEF2 Function in Mice Results in Spermatogenesis Defects and Primary Ciliary Dyskinesia. Biology of Reproduction 85, 690-701.

Yanagimachi, R., Wakayama, T., Kishikawa, H., Fimia, G.M., Monaco, L., and Sassone-Corsi, P. (2004). Production of fertile offspring from genetically infertile male mice. Proceedings of the National Academy of Sciences of the United States of America 101, 1691-1695.

Yanagisawa, H.-a., Mathis, G., Oda, T., Hirono, M., Richey, E.A., Ishikawa, H., Marshall, W.F., Kikkawa, M., and Qin, H. (2014). FAP20 is an inner junction protein of doublet microtubules essential for both the planar asymmetrical waveform and stability of flagella in Chlamydomonas. Molecular Biology of the Cell 25, 1472-1483.

Zhang, T., Li, S., Zhang, Y., Zhong, C., Lai, Z., and Ding, J. (2009a). Crystal structure of the ARL2-GTP-BART complex reveals a novel recognition and binding mode of small GTPase with effector. Structure 17, 602-610.

Zhang, Z., Kostetskii, I., Tang, W., Haig-Ladewig, L., Sapiro, R., Wei, Z., Patel, A.M., Bennett, J., Gerton, G.L., Moss, S.B., Radice, G.L., and Strauss, J.F., 3rd. (2006). Deficiency of SPAG16L causes male infertility associated with impaired sperm motility. Biol Reprod 74, 751759.

Zhang, Z., Sapiro, R., Kapfhamer, D., Bucan, M., Bray, J., Chennathukuzhi, V., McNamara, P., Curtis, A., Zhang, M., Blanchette-Mackie, E.J., and Strauss, J.F., 3rd. (2002). A spermassociated WD repeat protein orthologous to Chlamydomonas PF20 associates with Spag6, the mammalian orthologue of Chlamydomonas PF16. Molecular and cellular biology 22, 7993-8004.

Zhang, Z., Shen, X., Gude, D.R., Wilkinson, B.M., Justice, M.J., Flickinger, C.J., Herr, J.C., Eddy, E.M., and Strauss, J.F., 3rd. (2009b). MEIG1 is essential for spermiogenesis in mice. Proceedings of the National Academy of Sciences of the United States of America 106, 1705517060 .

Zhang, Z., Tang, W., Zhou, R., Shen, X., Wei, Z., Patel, A.M., Povlishock, J.T., Bennett, J., and Strauss, J.F. (2007). Accelerated mortality from hydrocephalus and pneumonia in mice with a combined deficiency of SPAG6 and SPAG16L reveals a functional interrelationship between the two central apparatus proteins. Cell Motility 64, 360-376. 
Zhou, C., Cunningham, L., Marcus, A.I., Li, Y., and Kahn, R.A. (2006). Arl2 and Arl3 regulate different microtubule-dependent processes. Mol Biol Cell 17, 2476-2487. 